\title{
ABUNDANCES IN A LARGE SAMPLE OF STARS IN M3 AND M13 ${ }^{1}$
}

\author{
Judith G. Cohen and Jorge Meléndez \\ Palomar Observatory, Mail Stop 105-24, California Institute of Technology, 1200 East California Boulevard, Pasadena, CA 91125; \\ jlc@astro.caltech.edu, jorge@astro.caltech.edu \\ Received 2004 July 19; accepted 2004 September 28
}

\begin{abstract}
We have carried out a detailed abundance analysis for 21 elements in a sample of 25 stars with a wide range in luminosity from luminous giants to stars near the main-sequence turnoff in the globular cluster M13 $([\mathrm{Fe} / \mathrm{H}]=$ $-1.50 \mathrm{dex})$ and in a sample of 13 stars distributed from the tip to the base of the red giant branch (RGB) in the globular cluster M3 $([\mathrm{Fe} / \mathrm{H}]=-1.39$ dex $)$. The analyzed spectra, obtained with HIRES at the Keck Observatory, are of high dispersion $(R=\lambda / \Delta \lambda=35,000)$. Most elements, including $\mathrm{Fe}$, show no trend with $T_{\mathrm{eff}}$ and low scatter around the mean between the top of the RGB and near the main-sequence turnoff, suggesting that at this metallicity, non-LTE effects and gravitationally induced heavy-element diffusion are not important for this set of elements over the range of stellar parameters spanned by our sample. We have detected an anticorrelation between $\mathrm{O}$ and $\mathrm{Na}$ abundances, observed previously among the most luminous RGB stars in both of these clusters, in both M3 and in M13 over the full range of luminosity of our samples, i.e., in the case of M13 to near the main-sequence turnoff. M13 shows a larger range in both $\mathrm{O}$ and $\mathrm{Na}$ abundance than does $\mathrm{M} 3$ at all luminosities, in particular having a few stars at its RGB tip with unusually strongly depleted $\mathrm{O}$. We detect a correlation between $\mathrm{Mg}$ abundance and $\mathrm{O}$ abundance among the stars in the M13 sample. We also find a decrease in the mean Mg abundance as one moves toward lower luminosity, which we tentatively suggest is due to our ignoring non-LTE effects in Mg. Although CN burning must be occurring in both $\mathrm{M} 3$ and in $\mathrm{M} 13$, and $\mathrm{ON}$ burning is required for M13, we combine our new $\mathrm{O}$ abundances with published $\mathrm{C}$ and $\mathrm{N}$ abundances to confirm with quite high precision that the sum of $\mathrm{C}+\mathrm{N}+\mathrm{O}$ is constant near the tip of the giant branch, and we extend this down to the bump in the luminosity function. The same holds true for a smaller sample in M3, with somewhat larger variance. Star I-5 in M13 has large excesses of Y and of $\mathrm{Ba}$, with no strong enhancement of $\mathrm{Eu}$, suggesting that an $s$-process event contributed to its heavy-element abundances. The mean abundance ratios for M3 and for M13 are identical to within the errors. They show the typical pattern for metal-poor globular clusters of scatter among the light elements, with the odd atomic number elements appearing in the mean enhanced. The Fe-peak elements, where the odd atomic number elements are excessively depleted, do not show any detectable star-to-star variations in either cluster. The abundance ratios for 13 Galactic globular clusters with recent detailed abundance analyses, obtained by combining our samples with published data, are compared with those of published large surveys of metal-poor halo field stars. For most elements, the agreement is very good, suggesting a common chemical history for the halo field and cluster stars.
\end{abstract}

Key words: globular clusters: general — globular clusters: individual (M3, M13) — stars: abundances stars: evolution

Online material: machine-readable tables

\section{INTRODUCTION}

Abundance determinations of stars in Galactic globular clusters can provide valuable information about important astrophysical processes, such as stellar evolution, stellar structure, Galactic chemical evolution, and the formation of the Milky Way. Surface stellar abundances of $\mathrm{C}, \mathrm{N}, \mathrm{O}$, and often Na, $\mathrm{Mg}$, and $\mathrm{Al}$, are found to be variable among red giants within a globular cluster. The physical process responsible for these star-to-star element variations is still uncertain (see the reviews of Kraft 1994 and Pinsonneault 1997, as well as Cohen et al. 2002 and Ventura et al. 2001).

\footnotetext{
${ }^{1}$ Some of the data presented herein were obtained at the W. M. Keck Observatory, which is operated as a scientific partnership among the California Institute of Technology, the University of California, and the National Aeronautics and Space Administration. The Observatory was made possible by the generous financial support of the W. M. Keck Foundation. The authors wish to recognize and acknowledge the very significant cultural role and reverence that the summit of Mauna Kea has always had within the indigenous Hawaiian community. We are most fortunate to have the opportunity to conduct observations from this mountain.
}

To study the origin of the star-to-star abundance variations, we started a program to determine chemical abundances of the nearer Galactic globular cluster stars. In previous papers in this series, we studied a sample of 25 stars in M71, the nearest globular cluster reachable from the northern hemisphere (Cohen et al. 2001; Ramírez et al. 2001; Ramírez \& Cohen 2002), followed by a similar-sized sample in M5, the nearest intermediatemetallicity globular cluster accessible from a northern hemisphere site (Ramírez \& Cohen 2003). Our sample in each cluster includes stars over a large range in luminosity, in order to study in a consistent manner red giants, horizontal-branch stars, and stars at the main-sequence turnoff. We measured the iron abundance and the abundance ratios for $\sim 20$ elements with respect to Fe in each case, using high-dispersion $(R=\lambda / \Delta \lambda=$ $35,000)$ optical spectra obtained with HIRES at the Keck Observatory. We found that the $[\mathrm{Fe} / \mathrm{H}]$ abundances $^{2}$ from both

\footnotetext{
${ }^{2}$ The standard nomenclature is adopted; the abundance of element $\mathrm{X}$ is given by $\epsilon(\mathrm{X})=N(\mathrm{X}) / N(\mathrm{H})$ on a scale where $N(\mathrm{H})=10^{12} \mathrm{H}$ atoms. Then $[\mathrm{X} / \mathrm{H}]=$ $\log [N(\mathrm{X}) / N(\mathrm{H})]-\log [N(\mathrm{X}) / N(\mathrm{H})]_{\odot}$, and similarly for $[\mathrm{X} / \mathrm{Fe}]$.
} 
$\mathrm{Fe}$ I and $\mathrm{Fe}$ II lines agree with each other and with earlier determinations and that the $[\mathrm{Fe} / \mathrm{H}]$ obtained from $\mathrm{Fe}$ I and $\mathrm{Fe}$ II lines is constant within the rather small uncertainties over the full range in effective temperature $\left(T_{\text {eff }}\right)$ and luminosity (Ramírez et al. 2001). We also found that the neutron capture, the iron peak, and the $\alpha$-element abundance ratios show no trend with $T_{\text {eff }}$ and low scatter around the mean between the top of the red giant branch (RGB) and near the main-sequence turnoff in each cluster. We detected an anticorrelation between $\mathrm{O}$ and $\mathrm{Na}$ abundances in our sample of members of M71, which extends to the main sequence. We observed a statistically significant correlation in M5 between $\mathrm{Al}$ and $\mathrm{Na}$ abundances extending to $M_{V}=$ +1.8 , fainter than the luminosity of the RGB bump in M5. We merged our data with data compiled from the literature for the $\mathrm{Na}-\mathrm{O}$ anticorrelation seen among globular cluster stars to find that the slope of this relation is the same for all clusters studied to date, but the amplitude of the effect varies from cluster to cluster.

In the present paper, we study a sample of stars in M3 and in M13, globular clusters of even lower metallicity than M5, again covering a wide range in luminosity. There are many photometric studies of these two clusters, as they are the classic second-parameter pair, having similar abundances yet very different horizontal-branch morphologies. Those used in the present work are noted in $\S 5$.

The most important characteristic of the globular cluster M13, as compared with M71 and M5, is that M13 is well known to show unusually large star-to-star differences in the abundance of $\mathrm{Al}, \mathrm{Mg}, \mathrm{Na}$, and $\mathrm{O}$ among its red giants (see, e.g., Kraft et al. 1997). A study of $C$ and $N$ variations among a large sample of M13 stars from the tip of the RGB to the main-sequence turnoff is given in Briley et al. (2002, 2004); large star-to-star differences in $\mathrm{C}$ and $\mathrm{N}$ abundances were found. $\mathrm{M} 3$, on the other hand, resembles M5 and M71 in being relatively inactive in this regard, although its metallicity is quite close to that of M13.

M3 and M13 have been the subject of several previous high-dispersion analyses, beginning with that of Cohen (1978). Sneden et al. (2004) have recently presented a high-dispersion study of 28 red giants in M3 to confirm the lower level of starto-star variations in this cluster in contrast to M13; they compare their results with the older work of their group on both M3 and M31; see, e.g., Kraft et al. (1997).

M3 is more distant than M5 or M71, and we made no effort to reach the main-sequence turnoff in this cluster, although our sample reaches far down the RGB. For M13, which is at a distance comparable to that of M5, we reach close to the mainsequence turnoff.

\section{OBSERVATIONS}

To the maximum extent possible, the observing strategy, the atomic data, and the analysis procedures used here are identical to those developed in our earlier papers on M71 and on M5 (Cohen et al. 2001; Ramírez et al. 2001; Ramírez \& Cohen 2002, 2003).

\subsection{The Stellar Sample}

Stars were chosen to span the range from the tip of the RGB to the base of the subgiant branch in M13 and to well below the RGB bump in M3. Both of these clusters lie far from the Galactic plane, and hence field-star contamination is minimal. The photometric database of Stetson et al. (1998) and Stetson (2000), which is described in considerable detail in Cohen et al. (2002), was used to verify that the selected stars lie on the cluster locus in various color-magnitude diagrams. This database, in the form available at the time, only included small sections of the solid angle required to fully cover each of these two clusters.

Pairs were selected in M3 for which both stars lie below the luminosity of the RGB bump and appear, based on their broadband colors, to be members. A separation of less than $9^{\prime \prime}$ was required. Only reasonably isolated stars were selected. To this we added a sample of bright giants, most of which were selected to span the full range in $\mathrm{Na}$ and/or $\mathrm{O}$ abundances from Kraft et al. (1992). Throughout this paper, the star names are from Sandage (1953) for the brightest stars in M3 or from von Zeipel (1908) for the two bright stars near the center of M3 not included in the former work, or for the stars previously not cataloged, they are assigned based on the object's J2000 coordinates, so that a star with right ascension, declination of $13 \mathrm{rm} \mathrm{rs.s,}+28 \mathrm{dm}$ ds is identified in this paper with the name Crmrss_dmds. For M13, the primary source of star identifications is Arp (1955; see also Kadla 1966). For the M13 stars not previously cataloged, names are assigned on the basis of the object's J2000 coordinates, so that a star with right ascension, declination of $16 \mathrm{rm} \mathrm{rs.s,}+36 \mathrm{dm}$ $\mathrm{ds}$ is identified in this paper with the name Crmrss_dmds.

Our sample in M3 totals 13 stars, including five on the RGB well below the luminosity of the HB, and one RHB star. Figure $1 a$ shows the sample in M3 superposed on a color-magnitude diagram of this globular cluster.

Eight pairs of stars were selected for observation in M13 using criteria similar to those used for the M3 pairs. Two of the 16 stars turned out not to be members of M13, and two others are horizontal-branch stars too hot to analyze. These members of M13 reach to $V=17.9$ mag. In addition, 13 bright giants were observed, chosen to have previous observations of $[\mathrm{C} / \mathrm{Fe}]$ and of $[\mathrm{N} / \mathrm{Fe}]$ from Suntzeff (1981) or from Smith et al. (1996). This sample includes stars covering the full range of $\mathrm{Na}$, $\mathrm{Mg}$, and Al abundances found in M13 by Kraft et al. (1997). Figure $1 b$ shows the sample in M13 superposed on a colormagnitude diagram of this globular cluster.

Our sample in M13 totals 25 stars reaching from the tip of the RGB to almost the main-sequence turnoff, plus two hot HB stars that we have not attempted to analyze.

\subsection{Data Acquisition and Reduction}

All spectra were obtained with HIRES (Vogt et al. 1994) at the Keck Observatory. In 2001 May we observed with an instrumental configuration similar to that used in our earlier globular cluster work. The wavelength range from 5500 to $7800 \AA$ was covered with gaps between the orders due to the undersized HIRES detector. We wanted to include key lines of critical elements, specifically the [O I] lines at 6300 and $6363 \AA$, the O triplet at $7770 \AA$, the Na doublet at 6154 and $6160 \AA$, and the Al I lines at 6696 and $6698 \AA$. However, it was impossible to create a single instrumental configuration that included all the desired spectral features in the wavelength range 6000-8000 $\AA$, and a single compromise configuration had to be adopted. In particular, although the Al I doublet at 6696 and $6698 \AA$ is the most useful feature of that element in this spectral region, we could not get it to fit into a single HIRES setting together with the O lines. A 1".1 wide slit, corresponding to a spectral resolution of 34,000 , was used. A maximum slit length of $14^{\prime \prime}$ can be used with this instrumental configuration without orders overlapping. However, we found that covering beyond $7000 \AA$, while highly desirable to reach the $\mathrm{O}$ I triplet, added little else of interest in the spectra of these low-metallicity stars. Thus, for the 2003 June observations (the spring 2002 run being totally lost to weather), we shifted the instrument configuration toward 

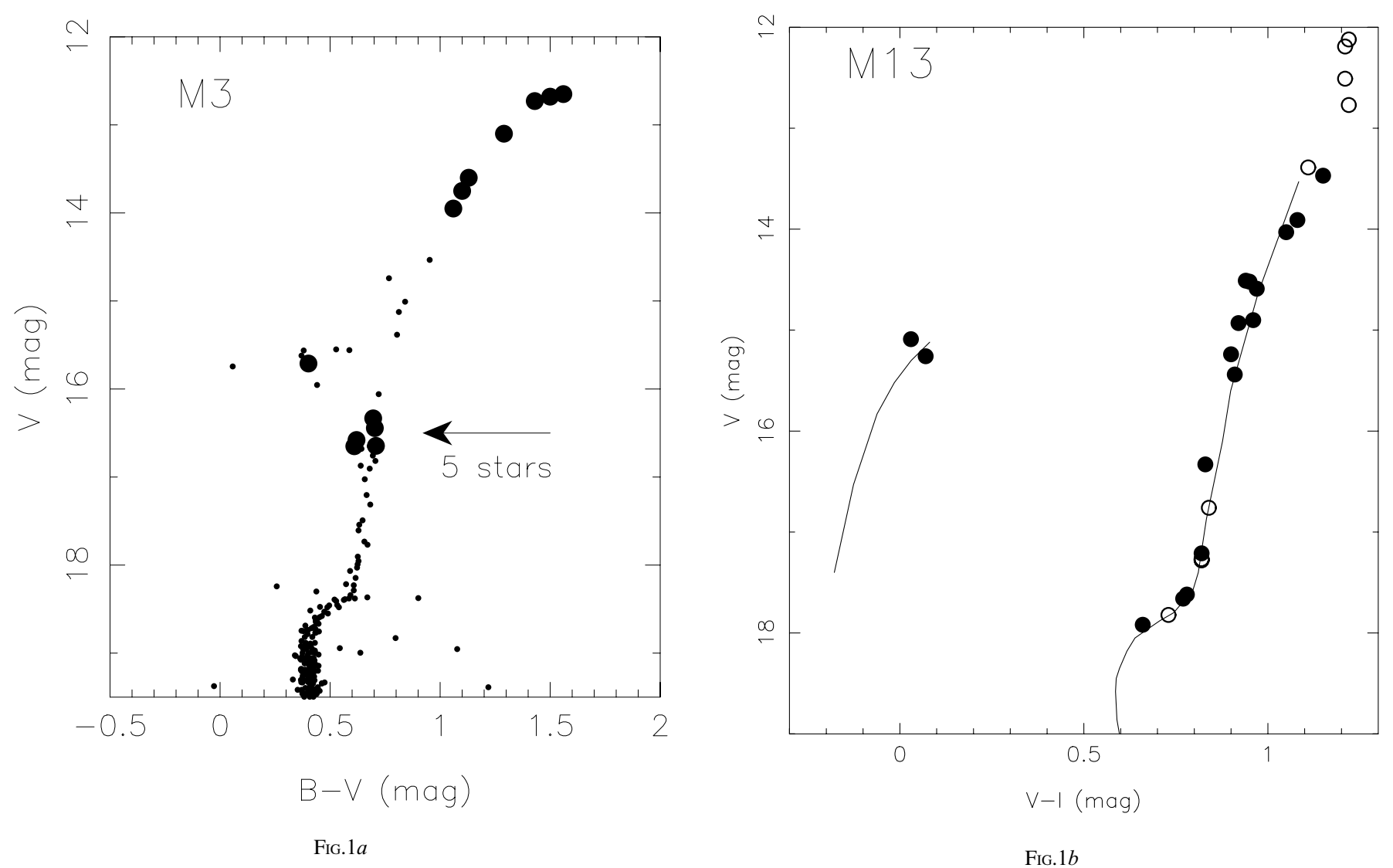

FIG. 1.-(a) $B-V$ color-magnitude diagram for a sector of M3 shown with data from the database of Stetson (2000). The 13 stars observed with HIRES are indicated by large filled circles. (b) $V-I$ locus for M13 from Johnson \& Bolte (1998) shown with the 27 cluster members observed with HIRES superposed (large circles). Open circles denote those sample stars without $I$ photometry from the database of Stetson (2000).

the blue, covering the range 4650-7010 $\AA$, again with small gaps between the orders. The maximum slit length without orders overlapping at the blue end of the spectrum is then reduced to 7 ". We call these two instrument configurations the "yellow" and the "red" configurations.

Since an image rotator for HIRES is available (built under the leadership of D. Tytler), if we can find pairs of program stars with suitable separations, they can be observed together on a single exposure. Pairs were preselected as described above to contain two members of the fainter M3 stars. In 2001 May, three (six) pairs of M3 (M13) stars ${ }^{3}$ were observed with HIRES using the "red" setting. One of the six stars in the M3 pairs turned out to be a red horizontal-branch star. In 2003 June, two of the pairs in M3 as well as seven bright giants were reobserved at higher signal-to-noise ratio $(\mathrm{S} / \mathrm{N})$ using the "yellow" setting; the third pair was too widely separated for this HIRES configuration. For M13, two of the pairs were reobserved in 2003 June or August, as were two new pairs as well as several bright giants with the "yellow" HIRES configuration.

The desired minimum $\mathrm{S} / \mathrm{N}$ was 75 over a 4 pixel resolution element for a wavelength near the center of the HIRES detector. This is calculated strictly from the counts in the object spectrum and excludes noise from cosmic-ray hits, sky subtraction, flattening problems, etc. Since the nights were relatively dark, sky subtraction is not an issue, except at the specific wavelengths corresponding to strong night-sky emission lines, such as the

\footnotetext{
${ }^{3}$ When values of a parameter are given simultaneously for both M3 and M13, the value for M3 is given, with that for M13 following in parentheses.
}

$\mathrm{Na} \mathrm{D}$ doublet. This $\mathrm{S} / \mathrm{N}$ goal was achieved for most of the stars reported here; see Table 1A for details regarding the faintest stars in the M13 sample. Note that for a fixed S/N in the continuum, for a star of a given luminosity, the lower metallicity of these clusters leads to weaker absorption lines, making it difficult to maintain the desired precision of the analysis. It is this that led to the repeat spectra with the "yellow" HIRES configuration.

The magnitude of the cluster radial velocity is large for both M3 and M13, and the cluster abundances are low. It was easy to tell after one integration whether or not a star is a member of the cluster. Approximate measurements of the radial velocity were made on line, and if a star was determined to be a nonmember, the observations were terminated. Very few nonmembers turned up in this way. If the probable nonmember was the second component in a pair, an attempt was made to switch to another position angle to pick up a different second star when a possible candidate that was bright enough was available within the maximum allowed separation. These data were reduced using a combination of Figaro scripts and the software package MAKEE. $^{4}$

Table 1B gives details of the HIRES exposures for each star, with the total exposure time for each object. All long integrations were broken up into separate exposures, each $1200 \mathrm{~s}$ long, to optimize cosmic-ray removal. The last column of the

\footnotetext{
${ }^{4}$ MAKEE was developed by T. A. Barlow specifically for reduction of Keck HIRES data. It is freely available on the World Wide Web at the Keck Observatory home page, http://www.2.keck.hawaii.edu/inst/hires/makeewww.
} 
TABLE $1 \mathrm{~A}$

The Sample of Stars in M13

\begin{tabular}{|c|c|c|c|c|c|}
\hline $\mathrm{ID}^{\mathrm{a}}$ & $\begin{array}{c}V^{\mathrm{b}} \\
(\mathrm{mag})\end{array}$ & Date Obs. & $\begin{array}{l}\text { Exp. Time } \\
\text { (s) }\end{array}$ & $\mathrm{S} / \mathrm{N}^{\mathrm{c}}$ & $\begin{array}{c}v_{r} \\
\left(\mathrm{~km} \mathrm{~s}^{-1}\right)\end{array}$ \\
\hline 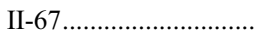 & 12.12 & 2003 Aug & 200 & $>100$ & -244.0 \\
\hline 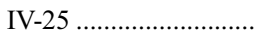 & 12.19 & 2003 Aug & 200 & $>100$ & -252.3 \\
\hline 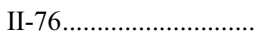 & 12.51 & 2003 Jun & 400 & $>100$ & -240.7 \\
\hline 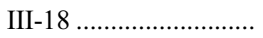 & 12.77 & 2003 Jun & 800 & $>100$ & -234.9 \\
\hline 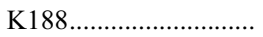 & 13.39 & 2003 Jun & 800 & 100 & -246.3 \\
\hline 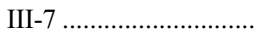 & 13.47 & 2003 Aug & 1200 & $>100$ & -261.5 \\
\hline 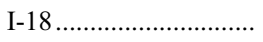 & 13.91 & 2003 Jun & 1000 & $>100$ & -247.1 \\
\hline 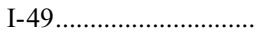 & 14.03 & 2003 Aug & 1200 & $>100$ & -261.2 \\
\hline 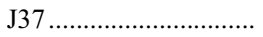 & 14.51 & 2003 Jun & 1200 & 95 & -249.3 \\
\hline C41196_2632 .............. & 14.52 & 2003 Aug & 2400 & $>100$ & d \\
\hline 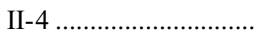 & 14.59 & 2003 Jun & 1200 & 100 & -241.3 \\
\hline 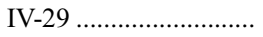 & 14.90 & 2003 Jun & 1800 & 100 & -245.1 \\
\hline 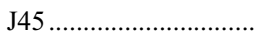 & 14.93 & 2003 Aug & 2400 & 100 & -243.7 \\
\hline C41155_3103 ............. & 15.24 & 2001 May & 3000 & $>100$ & -237.8 \\
\hline 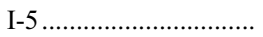 & 15.44 & $2003 \mathrm{Aug}$ & 2700 & 90 & -249.8 \\
\hline C41148_3103 ............. & 16.33 & 2001 May & 3000 & 80 & -235.7 \\
\hline C40559_2839............. & 16.76 & 2001 May & 1800 & 50 & -241.2 \\
\hline C41134_3056............. & 17.21 & 2003 Jun, Aug & 10800 & 90 & -256.6 \\
\hline C41134_3056............. & 17.21 & 2001 May & 8400 & 90 & -252.2 \\
\hline C41099_3046............. & 17.27 & 2003 Jun & 7200 & 85 & -252.6 \\
\hline C41099_3046............. & 17.27 & 2001 May & 6000 & 70 & -250.4 \\
\hline C40535_2813 ............. & 17.27 & 2001 May & 7200 & 65 & -244.9 \\
\hline C41101_3050 ............. & 17.28 & 2003 Jun & 7200 & 85 & -241.8 \\
\hline C41101_3050 ............ & 17.28 & 2001 May & 6000 & 65 & -239.8 \\
\hline C41135_3053 ….......... & 17.66 & 2003 Jun, Aug & 10800 & 75 & -248.7 \\
\hline C41135_3053 ............. & 17.66 & 2001 May & 8400 & 75 & -244.4 \\
\hline C41133_2750............. & 17.62 & 2001 May & 7200 & 65 & -237.2 \\
\hline C40535_2819 ............. & 17.82 & 2001 May & 7200 & 65 & -242.4 \\
\hline C41135_2753 ….......... & 17.92 & 2001 May & 7200 & 60 & -238.2 \\
\hline \multicolumn{6}{|l|}{ HB Stars: } \\
\hline C41195_2635 ......... & 15.1 & 2003 Aug & 2400 & 85 & \\
\hline C41265_2249......... & 15.9 & 2003 Aug & 1550 & 70 & \\
\hline
\end{tabular}

a Identifications are from Arp (1955) or Kadla (1966) or, if not included in the above, based on J2000 coordinates (Cmmsss_mmsss means R.A. $16 \mathrm{~mm}$ ss.s, decl. $+36 \mathrm{~mm}$ ss.s).

b $V$ photometry from Stetson's database (Stetson et al. 1998; Stetson 2000), from a 1990 update of Cudworth \& Monet (1979) (K. Cudworth 1993, private communication), or from Rey et al. (2001).

c $\mathrm{S} / \mathrm{N}$ in the continuum near $6380 \AA$ for the 2001 spectra and near $5865 \AA$ for the 2003 spectra per 4 pixel spectral resolution element.

d The value of $v_{r}$ for this star not measured because of technical issues. Star is a member of M13.

table gives the heliocentric radial velocity for each star, measured from the HIRES spectra; see Ramírez \& Cohen (2003) for the details of the procedure used to determine $v_{r}$. Based on their measured $v_{r}$, the 13 stars of our sample are all members of M3. They have a mean $v_{r}$ of $-147.6 \mathrm{~km} \mathrm{~s}^{-1}$, which agrees exactly with the value of Harris (1996). The velocity dispersion of our sample in M3 is $\sigma=4.7 \pm 1 \mathrm{~km} \mathrm{~s}^{-1}$, which, once the instrumental contribution is removed, is about $4.4 \mathrm{~km} \mathrm{~s}^{-1}$. This is comparable to the value of $5.6 \mathrm{~km} \mathrm{~s}^{-1}$ obtained by Gunn \& Griffin (1979) and by Pryor et al. (1988). For the 27 stars in M13 in our sample, the mean $v_{r}$ is $-245.7 \mathrm{~km} \mathrm{~s}^{-1}$, with $\sigma=7.1 \mathrm{~km}$ $\mathrm{s}^{-1}$. This is identical to the velocity dispersion obtained by Lupton et al. (1987) from a sample of more than 130 bright giants. Based on their HIRES spectra, stars C41262_2248 ( $V=$ 15.6) and C40560_2847 are not members of M13.

\section{EQUIVALENT WIDTHS}

The search for absorption features present in our HIRES data and the measurement of their equivalent width $\left(W_{\lambda}\right)$ was done automatically with a FORTRAN code, EWDET, developed for our globular cluster project. Details of this code and its features are described in Ramírez et al. (2001). Because M3 and M13 are considerably more metal-poor than M5 or M71, the determination of the continuum level was easier, and the equivalent widths measured automatically should be more reliable.

The initial list of unblended atomic lines and their atomic parameters was adopted from our work on M5, where it was created by inspection of the spectra of M5 stars, as well as the online solar spectrum taken with the FTS at the National Solar Observatory by Wallace et al. (1998) and the set of solar line identifications of Moore et al. (1966). The list of lines identified and measured by EWDET was then correlated, taking the radial velocity into account, to the template list of suitable unblended lines to specifically identify the various atomic lines. The automatic identifications were accepted as valid for lines with $W_{\lambda} \geq 15 \mathrm{~m} \AA$. They were checked by hand for all lines with smaller $W_{\lambda}$ and for all the rare earths. The equivalent widths of the $\mathrm{O}$ I lines were measured by hand, since they were generally very weak. The resulting $W_{\lambda}$ for $\sim 320$ lines in the spectra of the 13 stars in M3 are listed in Table 2A. The $W_{\lambda}$-values for the 25 stars in M13 are listed in Tables 2B and Table 2C. Note that lines with $W_{\lambda}>200 \mathrm{~m} \AA$ are not generally tabulated and 
TABLE 1B

The Sample of Stars in M3

\begin{tabular}{|c|c|c|c|c|c|}
\hline $\mathrm{ID}^{\mathrm{a}}$ & $\begin{array}{c}V^{\mathrm{b}} \\
(\mathrm{mag})\end{array}$ & Date of Obs. & $\begin{array}{l}\text { Exp. Time } \\
\text { (s) }\end{array}$ & $\mathrm{S} / \mathrm{N}^{\mathrm{c}}$ & $\begin{array}{c}v_{r} \\
\left(\mathrm{~km} \mathrm{~s}^{-1}\right)\end{array}$ \\
\hline 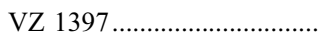 & 12.65 & 2003 Jun & 600 & $>100$ & -146.1 \\
\hline II-46 & 12.68 & 2003 Jun & 600 & $>100$ & -151.4 \\
\hline III-28 .................................... & 12.73 & 2003 Jun & 800 & $>100$ & -154.0 \\
\hline VZ $1000 \ldots \ldots \ldots$ & 13.01 & 2003 Jun & 400 & $>100$ & -151.8 \\
\hline IV-25 & 13.60 & 2003 Jun & 1000 & $>100$ & -150.4 \\
\hline C41303_2217......................... & 13.75 & 2003 Jun & 1000 & $>100$ & -144.5 \\
\hline IV-27 ................................. & 13.95 & 2003 Jun & 1000 & $>100$ & -151.4 \\
\hline C41543_2334......................... & 15.71 & 2001 May & 4800 & $>100$ & -139.9 \\
\hline C41543_2334 ...................... & 15.71 & 2003 Jun & 7200 & 100 & -145.7 \\
\hline 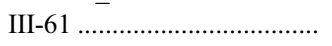 & 16.33 & 2001 May & 3600 & 80 & -148.7 \\
\hline III-60 ..................... & 16.44 & 2001 May & 3600 & 75 & -141.4 \\
\hline V-30 & 16.58 & 2001 May & 4800 & 85 & -143.6 \\
\hline V-30 & 16.58 & 2003 Jun & 8400 & 90 & -143.7 \\
\hline C41544_2336..................... & 16.65 & 2001 May & 4800 & 80 & -150.4 \\
\hline C41544_2336................... & 16.65 & 2003 Jun & 7200 & 90 & -153.8 \\
\hline V-31 & 16.65 & 2001 May & 4800 & 80 & -138.6 \\
\hline V-31 & 16.65 & 2003 Jun & 8400 & 90 & -149.8 \\
\hline
\end{tabular}

${ }^{a}$ Identifications are from Sandage (1953) or from von Zeipel (1908), or are based on J2000 coordinates (Cmmsss_mmsss means R.A. $13 \mathrm{~mm}$ ss.s, decl. $+28 \mathrm{~mm}$ ss.s).

b $V$ photometry from Sandage (1953), Buonanno et al. (1994), Ferraro et al. (1997), and Stetson (2000).

${ }^{c} \mathrm{~S} / \mathrm{N}$ in the continuum near $6380 \AA$ for the 2001 spectra and near $5865 \AA$ for the 2003 spectra per 4 pixel spectral-resolution element.

are not used unless there are no other available lines of that species.

\section{ATOMIC PARAMETERS}

The provenance of the $g f$-values and damping constants we adopt in our analysis of M3 and M13 stars is discussed below. In general, the atomic data and the analysis procedures used here are identical to those developed in our recent paper on M5 (Ramírez \& Cohen 2003).

\subsection{Transition Probabilities}

Transition probabilities for the Fe I lines were obtained from several laboratory experiments, including studies of Fe I absorption lines produced by iron vapor in a carbon tube furnace (Blackwell et al. 1979, 1982a, 1982b, 1986) (Oxford Group), measurement of radiative lifetimes of $\mathrm{Fe}$ I transitions by laserinduced fluorescence (O’Brian et al. 1991; Bard et al. 1991; Bard $\&$ Kock 1994), Fe I emission-line spectroscopy from a lowcurrent arc (May et al. 1974), and emission lines of Fe I from a shock tube (Wolnik et al. 1971). We also considered solar $g f$-values from Thévenin $(1989,1990)$ when needed. The Fe I $g f$-values obtained by the different experiments were placed into a common scale with respect to the results from O'Brian et al. (1991) (see Ramírez et al. 2001 for details). The $g f$-values for our Fe II lines were taken from the solar analysis of Blackwell et al. (1980), Biémont et al. (1991), and from the semiempirical calculations of Kurucz (1993b). For Fe I and Fe II $g f$-values, we used the same priority order for the $g f$-values from different experiments as in Ramírez et al. (2001).

Transition probabilities for the lines of atomic species other than iron were obtained from the NIST Atomic Spectra Database (NIST Standard Reference Database No. 78; see Weise et al. 1969, 1996; Martin et al. 1988; Fuhr et al. 1988) when possible. Nearly $80 \%$ of the lines selected as suitable from the HIRES spectra have transition probabilities from the NIST database. For the remaining lines the $g f$-values come from the inverted solar analysis of Thévenin (1989, 1990), with the exception of La II, Nd II, and Eu II lines, for which we have updated our values to those of Lawler et al. $(2001,2001)$ and Den Hartog et al. (2003).

Six elements show hyperfine structure splitting (Sc II, V I, $\mathrm{Mn} \mathrm{I}, \mathrm{Co} \mathrm{I}, \mathrm{Cu}$ I, and $\mathrm{Ba}$ II). The corresponding hyperfine structure constants were taken from Prochaska et al. (2000). For Ba II, we adopt the HFS from McWilliam (1998). We use the laboratory spectroscopy of Lawler et al. $(2001,2001)$ to calculate the HFS patterns for La II and for Eu II.

We use the damping constants of Barklem et al. (2000), which were calculated on the basis of the theory of Anstee, Barklem, and O'Mara (Barklem et al. 1998), when available. If there is no entry in their database, as in our earlier work, the damping constants were set to twice that of the Unsöld approximation for van der Waals broadening, following Holweger et al. (1991).

\subsection{Solar Abundances}

The regime in which we are operating is so metal-poor that we cannot in general attempt to calculate solar abundances corresponding to our particular choices of atomic data because the lines seen in these metal-poor globular cluster stars are far too strong in the Sun. We must therefore rely on the accuracy of the $g f$-values for each element across the large relevant range of line strength and wavelength. We adopt the solar abundances of Anders \& Grevesse (1989) for most elements. For Ti and for $\mathrm{Sr}$, we adopt the slightly modified values given in Grevesse \& Sauval (1998). For the special cases of La II, Nd II, and Eu II we use the results found by the respective recent laboratory studies cited above. For $\mathrm{Mg}$, we adopt the slightly updated value suggested by Holweger (2001), ignoring the small suggested nonLTE and granulation corrections, since we do not implement such in our analyses.

There is considerable controversy regarding the abundances of the CNO elements in the Sun, with the recent results of 
TABLE 2A

Equivalent Widths For the M3 Stars

\begin{tabular}{|c|c|c|c|c|c|c|c|c|c|c|c|c|c|c|c|c|}
\hline Ion & $\begin{array}{c}\lambda \\
(\AA)\end{array}$ & $\begin{array}{c}\chi \\
(\mathrm{eV})\end{array}$ & $\log g f$ & $\begin{array}{c}\text { VZ } 1397 \\
(\mathrm{~m} \AA)\end{array}$ & $\begin{array}{l}\text { II } 46 \\
\text { (mÅ) }\end{array}$ & $\begin{array}{l}\text { III } 28 \\
\text { (mÅ) }\end{array}$ & $\begin{array}{l}\mathrm{VZ} 1000 \\
(\mathrm{~m} \AA)\end{array}$ & $\begin{array}{l}\text { IV } 25 \\
(\mathrm{~m} \AA)\end{array}$ & $\begin{array}{c}\text { C41303_2217 } \\
(\mathrm{m} \AA)\end{array}$ & $\begin{array}{l}\text { IV } 27 \\
(\mathrm{~m} \AA)\end{array}$ & $\begin{array}{c}\text { C41543_2334 } \\
(\mathrm{m} \AA)\end{array}$ & $\begin{array}{l}\text { III } 61 \\
(\mathrm{~m} \AA)\end{array}$ & $\begin{array}{l}\text { III } 60 \\
(\mathrm{~m} \AA)\end{array}$ & $\begin{array}{l}\text { V } 30 \\
(\mathrm{~m} \AA)\end{array}$ & $\begin{array}{c}\text { C41544_2336 } \\
(\mathrm{m} \AA)\end{array}$ & $\begin{array}{l}\text { V } 31 \\
(\mathrm{~m} \AA)\end{array}$ \\
\hline $\mathrm{O}_{\text {I }}$ & 6300.30 & 0.00 & -9.78 & 77.2 & 66.5 & 55.0 & 28.5 & 39.0 & 37.0 & 16.0 & $\ldots$ & $\ldots$ & $\ldots$ & $\ldots$ & $\ldots$ & \\
\hline 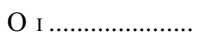 & 6363.78 & 0.02 & -10.30 & 41.0 & 27.5 & 28.1 & 14.6 & 14.0 & 15.7 & 7.0 & & & & & $\ldots$ & \\
\hline 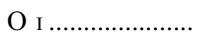 & 7771.94 & 9.15 & 0.37 & $\ldots$ & $\ldots$ & $\ldots$ & $\ldots$ & $\ldots$ & $\ldots$ & $\ldots$ & 111.0 & 32.0 & 27.0 & $\leq 14.0$ & 32.0 & 22.0 \\
\hline $\mathrm{O}_{\mathrm{I}} \ldots \ldots \ldots \ldots \ldots \ldots$ & 7774.17 & 9.15 & 0.22 & $\ldots$ & $\ldots$ & $\ldots$ & $\ldots$ & $\ldots$ & $\ldots$ & $\ldots$ & 90.0 & 30.0 & 20.0 & $\ldots$ & 25.0 & $\ldots$ \\
\hline $\mathrm{O}_{\mathrm{r}} \ldots \ldots \ldots \ldots \ldots \ldots$ & 7775.39 & 9.15 & 0.00 & $\ldots$ & $\ldots$ & $\ldots$ & $\ldots$ & $\ldots$ & $\ldots$ & $\ldots$ & 71.0 & $\ldots$ & $\ldots$ & $\ldots$ & $\ldots$ & $\ldots$ \\
\hline 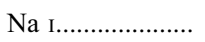 & 5682.63 & 2.10 & -0.70 & 72.1 & 58.0 & 30.2 & 73.9 & 46.3 & 33.0 & 55.9 & $\ldots$ & $\ldots$ & $\ldots$ & 41.0 & 23.0 & 19.0 \\
\hline
\end{tabular}

NoтE.-Table 2A is presented in its entirety in the electronic edition of the Astronomical Journal. A portion is shown here for guidance regarding its form and content.

TABLE 2B

Equivalent Widths For the Brighter M13 Stars

\begin{tabular}{|c|c|c|c|c|c|c|c|c|c|c|c|c|c|c|c|c|}
\hline Ion & $\begin{array}{c}\lambda \\
(\AA)\end{array}$ & $\begin{array}{c}\chi \\
(\mathrm{eV})\end{array}$ & $\log g f$ & $\begin{array}{l}\text { II } 67 \\
(\mathrm{~m} \AA)\end{array}$ & $\begin{array}{l}\text { IV } 25 \\
(\mathrm{~m} \AA)\end{array}$ & $\begin{array}{l}\text { II } 76 \\
(\mathrm{~m} \AA)\end{array}$ & $\begin{array}{l}\text { III } 18 \\
(\mathrm{~m} \AA)\end{array}$ & $\begin{array}{l}\text { K } 18 \\
(\mathrm{~m} \AA)\end{array}$ & $\begin{array}{l}\text { III } \\
(\mathrm{m} \AA)\end{array}$ & $\begin{array}{l}\text { I } 18 \\
(\mathrm{~m} \AA)\end{array}$ & $\begin{array}{l}\text { I } 49 \\
(\mathrm{~m} \AA)\end{array}$ & $\begin{array}{l}\mathrm{J} 37 \\
(\mathrm{~m} \AA)\end{array}$ & $\begin{array}{c}\text { C41196_2632 } \\
\text { (mA) }\end{array}$ & 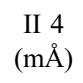 & $\begin{array}{l}\text { IV } 29 \\
(\mathrm{~m} \AA)\end{array}$ & $\begin{array}{l}\mathrm{J} 45 \\
(\mathrm{~m} \AA)\end{array}$ \\
\hline $\mathrm{O}_{\mathrm{I}}$ & 6300.30 & 0.00 & -9.78 & 6.1 & 20.0 & 55.0 & 10.0 & 26.0 & 30.0 & 16.0 & 30.0 & 9.0 & 6.5 & 10.0 & 14.0 & 7.0 \\
\hline $\mathrm{O}_{\mathrm{I}}$ & 6363.78 & 0.02 & -10.30 & $\ldots$ & 7.0 & 23.8 & $\ldots$ & 11.7 & 14.0 & $\ldots$ & 9.0 & $\ldots$ & $\ldots$ & $\ldots$ & $\ldots$ & $\ldots$ \\
\hline 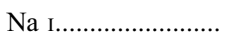 & 5682.63 & 2.10 & -0.70 & 109.2 & 112.0 & 30.0 & 83.7 & 36.0 & 40.7 & 55.0 & 39.4 & 56.0 & 42.3 & 43.5 & 15.5 & 41.0 \\
\hline 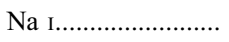 & 5688.19 & 2.10 & -0.42 & 124.0 & 138.0 & 52.8 & 106.4 & 59.0 & 57.8 & 80.4 & 53.0 & 75.6 & 61.7 & 63.4 & 29.4 & 57.3 \\
\hline 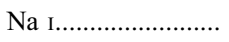 & 6154.23 & 2.10 & -1.53 & 45.7 & 46.9 & 6.6 & 27.0 & 10.0 & $\ldots$ & 14.5 & $\ldots$ & 19.0 & $\ldots$ & 8.5 & $\ldots$ & $\ldots$ \\
\hline $\mathrm{Na}$ & 6160.75 & 2.00 & -1.23 & $\ldots$ & 67.9 & 11.0 & 44.0 & 18.1 & $\ldots$ & 29.6 & 17.0 & 23.5 & 20.0 & 21.0 & $\ldots$ & 16.0 \\
\hline
\end{tabular}

Noте.-Table 2B is presented in its entirety in the electronic edition of the Astronomical Journal. A portion is shown here for guidance regarding its form and content.

TABLE 2C

Equivalent Widths for the Fainter M13 Stars

\begin{tabular}{|c|c|c|c|c|c|c|c|c|c|c|c|c|c|c|c|}
\hline Ion & $\begin{array}{c}\lambda \\
(\AA)\end{array}$ & $\begin{array}{c}\chi \\
(\mathrm{eV})\end{array}$ & $\log g f$ & $\begin{array}{c}\text { I } 5 \\
(\mathrm{~m} \AA)\end{array}$ & $\begin{array}{c}\text { C41155_3103 } \\
(\mathrm{m} \AA)\end{array}$ & $\begin{array}{c}\text { C41148_3103 } \\
(\mathrm{mÅ})\end{array}$ & $\begin{array}{c}\text { C41134_3056 } \\
(\mathrm{m} \AA)\end{array}$ & $\begin{array}{c}\text { C40559_2839 } \\
(\mathrm{m} \AA)\end{array}$ & $\begin{array}{c}\mathrm{C} 41101 \_3050 \\
(\mathrm{mÅ})\end{array}$ & $\begin{array}{c}\text { C41099_3046 } \\
(\mathrm{m} \AA)\end{array}$ & $\begin{array}{c}\text { C41135_3053 } \\
(\mathrm{m} \AA)\end{array}$ & $\begin{array}{c}\text { C41133_2750 } \\
(\mathrm{m} \AA)\end{array}$ & $\begin{array}{c}\text { C40535_2819 } \\
(\mathrm{mÅ})\end{array}$ & $\begin{array}{c}\text { C40539_2813 } \\
(\mathrm{mA})\end{array}$ & $\begin{array}{c}\text { C41135_2753 } \\
(\mathrm{mÅ})\end{array}$ \\
\hline O г ...... & 7771.94 & 9.15 & 0.37 & $\ldots$ & 12.0 & 34.0 & 16.0 & 25.0 & 22.5 & 20.0 & 20.0 & 20.0 & 25.0 & 19.0 & 16.5 \\
\hline $\mathrm{O}_{\mathrm{I}} \ldots . . .$. & 7774.17 & 9.15 & 0.22 & $\ldots$ & $\ldots$ & 25.0 & 14.0 & 17.0 & $\ldots$ & 15.0 & 18.0 & 14.0 & 25.0 & 19.0 & 20.0 \\
\hline $\mathrm{Na}$ I..... & 5682.63 & 2.10 & -0.70 & 56.0 & 49.0 & 20.0 & 21.7 & 26.0 & 30.3 & 29.0 & 14.0 & $\ldots$ & 19.0 & 20.0 & \\
\hline $\mathrm{Na}$ I..... & 5688.19 & 2.10 & -0.42 & 76.5 & 69.0 & 29.0 & 44.3 & 33.0 & 44.3 & 48.0 & 23.3 & 27.0 & 26.0 & 35.0 & 27.0 \\
\hline $\mathrm{Na}$ I..... & 6160.75 & 2.00 & -1.23 & 26.6 & 22.0 & $\ldots$ & $\ldots$ & $\ldots$ & $\ldots$ & $\ldots$ & $\ldots$ & $\ldots$ & $\ldots$ & $\ldots$ & $\ldots$ \\
\hline
\end{tabular}

Note.-Table $2 \mathrm{C}$ is presented in its entirety in the electronic edition of the Astronomical Journal. A portion is shown here for guidance regarding its form and content. 
Allende Prieto et al. (2002), Asplund (2003), and Asplund et al. (2004) being considerably ( $\sim 0.2$ dex $)$ lower than those of Anders \& Grevesse (1989) and somewhat lower than those of Grevesse \& Sauval (1998). We have derived an inverse solar $\mathrm{O}$ abundance for our particular choice of atomic data and of model atmospheres using both the forbidden doublet at 6300 , $6363 \AA$ and the triplet at $7770 \AA$. The equivalent widths of these lines in the Sun were measured from the solar atlas of Kurucz et al. (1984) and checked in the McDonald Observatory solar spectrum used by Allende Prieto et al. (2004). Corrections for the contribution of the $\mathrm{Ni}$ I line to the line at $6300 \AA$ (Allende Prieto et al. 2001) and the $\mathrm{CN}$ lines to the line at $6363 \AA$ (Asplund et al. 2004) were made. We adopted the $\mathrm{O}$ abundance from [O $\mathrm{I}$; after applying a non-LTE correction interpolated from the calculations of Gratton et al. (1999), the $\mathrm{O}$ abundance from the triplet lines is only 0.05 dex smaller than derived from the forbidden lines.

We adopt $\log \epsilon(\mathrm{Fe})=7.45$ dex for iron, following the revisions in the solar photospheric abundances suggested by Asplund et al. (2000) and Holweger (2001). This value is somewhat lower than that given by Grevesse \& Sauval (1998) and considerably lower than that recommended by Anders \& Grevesse (1989). Some papers in the literature use the Grevesse \& Sauval (1998) value, and some older ones use $7.67 \mathrm{dex}$, the value recommended by Anders \& Grevesse (1989). In such cases, their values of $[\mathrm{Fe} / \mathrm{H}]$ will be 0.1 to 0.2 dex smaller than ours, while their abundance ratios $[\mathrm{X} / \mathrm{Fe}]$ are the same amount larger than ours.

Table 3 gives the solar abundances used here.

\section{STELLAR PARAMETERS}

We follow the philosophy developed in our earlier work on globular cluster stars and described in Cohen et al. (2001). $T_{\text {eff }}$ is derived by comparing reddening-corrected broadband colors with the predictions of grids of model atmospheres. Here we use the grid of predicted broadband colors and bolometric corrections of Houdashelt et al. (2000), based on the MARCS stellar atmosphere code of Gustafsson et al. (1975). In Cohen et al. (2001) we demonstrated that the Kurucz and MARCS predicted colors are essentially identical, at least for the specific colors used here. We adopt current values from the online database of Harris (1996) for the distances of 10.4 (7.5) kpc for M3 (M13) with a reddening of $E(B-V)=0.01(0.02) \mathrm{mag}$. The relative extinction in various passbands is taken from Cohen et al. (1981) (see also Schlegel et al. 1998). On the basis of the earlier high-dispersion analyses of Kraft et al. (1997) and Sneden et al. (2004), we adopt as an initial guess $[\mathrm{Fe} / \mathrm{H}]=-1.5 \mathrm{dex}$ for these two globular clusters.

Our primary source for optical photometry is the $B V I$ database of Stetson (Stetson et al. 1998; Stetson 2000). All the bright giants in M3, except VZ 1000 and VZ 1397, which are too close to the center of this cluster, are included there. All components of the pairs except V-30+V-31, which are too far from the center of M3, are included as well. Photometry for the missing stars was obtained from Sandage (1953), Johnson \& Sandage (1956), Buonanno et al. (1994), or Ferraro et al. (1997), with preference given to the most recent study.

For M13, we used (in order of preference) the optical photometry of Cudworth (1979), Rey et al. (2001), Buonanno et al. (1994), and Stetson (Stetson et al. 1998; Stetson 2000). After a small adjustment in the zero point of the (uncalibrated) photometry of Buonanno et al. (1994), the agreement between the various data sets is reasonable. The area in M13 covered by the work of Johnson \& Bolte (1998), Rosenberg et al. (2000), and
TABLE 3

Adopted Solar Abundances

\begin{tabular}{|c|c|c|c|}
\hline Element & {$[\mathrm{X} / \mathrm{H}]^{\mathrm{a}}$} & Element & {$[\mathrm{X} / \mathrm{H}]^{\mathrm{a}}$} \\
\hline О & 8.85 & 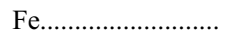 & 7.45 \\
\hline $\mathrm{Na}$ & 6.33 & 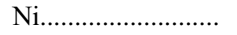 & 6.25 \\
\hline $\mathrm{Mg}$ & 7.54 & $\mathrm{Cu}$ & 4.21 \\
\hline $\mathrm{Al}$ & 6.47 & $\mathrm{Zn}$ & 4.60 \\
\hline 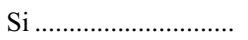 & 7.55 & $\mathrm{Ba}$ & 2.13 \\
\hline $\mathrm{Ca}$ & 6.36 & Y & 2.24 \\
\hline 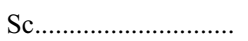 & 3.10 & 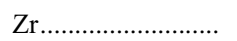 & 2.60 \\
\hline 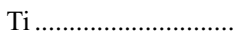 & 4.99 & $\mathrm{La}$ & 1.14 \\
\hline V & 4.00 & $\mathrm{Nd}$ & 1.45 \\
\hline 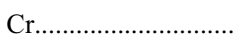 & 5.67 & Eu & 0.51 \\
\hline $\mathrm{Mn}$ & 5.39 & 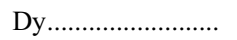 & 1.10 \\
\hline
\end{tabular}

${ }^{\text {a }}$ Given on a scale for which $\log [N(\mathrm{H})]=12.0$; values in dex.

by Piotto et al. (2002) does not overlap with our stars; nor does that of the IR photometry of Davidge \& Courteau (1999) and of Valenti et al. (2004).

We only used $V$ and $I$ magnitudes from these sources, combining them with $J$ and $K$ photometry from 2MASS (Skrutskie et al. 1997; Cutri et al. 2003). This worked extremely well for the bright giants, with a scatter in deduced $T_{\text {eff }}$ from $V-I$, $V-J$, and $V-K$ of under $40 \mathrm{~K}$. The fainter stars in our sample (i.e., the pairs) show much larger scatter in their deduced $T_{\text {eff }}$ from the various colors. These stars are too crowded (and in some cases rather faint) for 2MASS, which uses an aperture size of $2^{\prime \prime}$. This problem is more serious in M13, as our sample reaches fainter there than in M3; a large fraction of the pairs observed in M13 had no entry or only a single entry in the 2MASS database.

To get around this problem, in 2004 April we observed the fields of the fainter stars in our samples in both M3 and M13 with the Wide Field Infrared Camera (WIRC; Wilson et al. 2003) at the $5 \mathrm{~m}$ Hale Telescope for the purpose of establishing reliable $J, K$ magnitudes for the fainter stars in our sample. The 2MASS colors of nearby isolated somewhat brighter stars were used to calibrate our WIRC photometry. This new infrared photometry for only the pairs in M3 and M13 is listed in Table 4 . The agreement with 2MASS is very good for the brighter, wider pairs.

With improved infrared photometry in hand, the observed broadband colors $V-I, V-J$, and $V-K$ for each program star from the sources listed above, corrected for extinction, were used to determine $T_{\text {eff }}$. The set of models with metallicity of $-1.5 \mathrm{dex}$, nearest to our initial estimate of $[\mathrm{Fe} / \mathrm{H}]$, is used. Table 5A (Table 5B) lists the $T_{\text {eff }}$ thus deduced for the sample in M3 and in M13. The reddening to each of these clusters is small, making possible extinction variations across the cluster irrelevant. We assume a random photometric error of $0.02 \mathrm{mag}$ applies to $V-I$ from Stetson (2000). Following Cohen et al. (2001), this translates into a total uncertainty in $T_{\text {eff }}$ of $75 \mathrm{~K}$ for giants rising to $150 \mathrm{~K}$ for main-sequence stars using only $V-I$. The errors in $T_{\text {eff }}$ deduced from $V-J$ or $V-K$ are about a factor of 2 smaller.

We have slightly smoothed the $T_{\text {eff }}$ for the fainter stars in our sample by small amounts to ensure that stars at approximately the same evolutionary stage have approximately the same stellar parameters. Once an initial guess at $T_{\text {eff }}$ has been established from a broadband color, it is possible with minimal assumptions to evaluate $\log (g)$ using observational data. The adopted distance modulus for the cluster, initial guess at $T_{\text {eff }}$ for the star, 
TABLE 4

New IR Photometry for the Fainter Stars

\begin{tabular}{|c|c|c|}
\hline $\mathrm{ID}^{\mathrm{a}}$ & $\begin{array}{c}J \\
(\mathrm{mag})^{\mathrm{b}}\end{array}$ & $\begin{array}{c}K \\
(\mathrm{mag})^{\mathrm{b}}\end{array}$ \\
\hline \multicolumn{3}{|c|}{ M3 } \\
\hline C41544_2336_................... & 15.16 & 14.68 \\
\hline C41543_2334_.................... & 14.67 & 14.38 \\
\hline III-60 & 15.03 & 14.52 \\
\hline 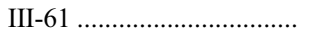 & 14.92 & 14.41 \\
\hline V-31 & 15.33 & 14.74 \\
\hline 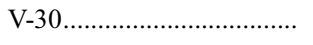 & 15.18 & 14.61 \\
\hline \multicolumn{3}{|c|}{ M13 } \\
\hline C41134_3056 ..................... & 15.73 & 15.32 \\
\hline C41135_3053 ….................. & 16.29 & 15.91 \\
\hline C41101_3050_.................... & 15.88 & 15.40 \\
\hline C41099_3046................... & 15.88 & 15.44 \\
\hline C40539_2813 ..................... & 16.49 & 16.08 \\
\hline C40535_2819................... & 16.58 & 16.19 \\
\hline C41135_2753 …................. & 16.87 & 16.44 \\
\hline C41133_2750 ................... & 16.30 & 15.86 \\
\hline C41148_3103 …................... & 14.87 & 14.30 \\
\hline C41155_3103 …................... & 13.64 & 13.09 \\
\hline C40559_2839.................... & 12.84 & 12.20 \\
\hline C41196_2632_.................... & 12.84 & 12.20 \\
\hline C41195_2635 ….................. & 14.72 & 14.82 \\
\hline
\end{tabular}

and an assumed stellar mass (we adopt $0.8 M_{\odot}$ for the stars in M3 and in M13) are combined with the known interstellar absorption, the bolometric corrections predicted by the model atmosphere grid, as well as a broadband observed $V$ magnitude to calculate $\log (g)$. An iterative scheme is used to correct for the small dependence of the predictions of the model atmosphere grid on $\log (g)$ itself. Rapid convergence is achieved.

It is important to note that because of the constraint of a known distance to each cluster, the uncertainty in $\log (g)$ for any star in our sample is small, $\leq 0.1 \mathrm{dex}$, when comparing two members of the same cluster. Propagating an uncertainty of $15 \%$ in the cluster distance, $5 \%$ in the stellar mass, and a gen-

TABLE 5A

Stellar Parameters for the M3 Sample

\begin{tabular}{|c|c|c|c|}
\hline $\mathrm{ID}^{\mathrm{a}}$ & $\begin{array}{l}T_{\text {eff }} \\
(\mathrm{K})\end{array}$ & $\begin{array}{l}\log (g) \\
(\operatorname{dex})\end{array}$ & $\begin{array}{c}v_{t} \\
\left(\mathrm{~km} \mathrm{~s}^{-1}\right)\end{array}$ \\
\hline 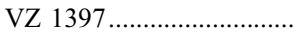 & 3985 & 0.40 & 1.90 \\
\hline II-46 .................................. & 3998 & 0.40 & 1.90 \\
\hline 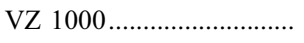 & 4175 & 0.70 & 1.85 \\
\hline III-28 & 4200 & 0.60 & 1.80 \\
\hline IV-25 & 4408 & 1.10 & 1.60 \\
\hline C41303_2217....................... & 4436 & 1.20 & 1.60 \\
\hline IV-27 & 4547 & 1.30 & 1.60 \\
\hline III-61 & 5140 & 2.70 & 1.35 \\
\hline III-60 ................................. & 5170 & 2.65 & 1.30 \\
\hline C41544_2336.................... & 5170 & 2.70 & 1.25 \\
\hline V-30 & 5170 & 2.75 & 1.40 \\
\hline V-31 & 5170 & 2.80 & 1.20 \\
\hline C41543_2334b ...................... & 6090 & 2.70 & 2.05 \\
\hline
\end{tabular}

${ }^{\text {a }}$ Identifications as in notes to Table 1A.

b This is a RHB star.
TABLE 5B

Stellar Parameters for the M13 Sample

\begin{tabular}{|c|c|c|c|}
\hline $\mathrm{ID}^{\mathrm{a}}$ & $\begin{array}{l}T_{\text {eff }} \\
(\mathrm{K})\end{array}$ & $\begin{array}{c}\log (g) \\
(\text { dex })\end{array}$ & $\begin{array}{c}v_{t} \\
\left(\mathrm{~km} \mathrm{~s}^{-1}\right)\end{array}$ \\
\hline 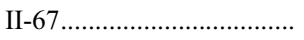 & 3900 & 0.45 & 1.9 \\
\hline IV-25 .................................. & 3985 & 0.50 & 1.95 \\
\hline II-76 & 4300 & 0.85 & 1.95 \\
\hline 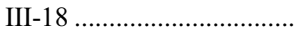 & 4350 & 1.00 & 1.65 \\
\hline К188 & 4535 & 1.37 & 1.45 \\
\hline 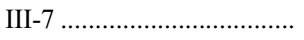 & 4550 & 1.40 & 1.35 \\
\hline I-18 & 4690 & 1.65 & 1.5 \\
\hline I-49.1. & 4745 & 1.75 & 1.55 \\
\hline 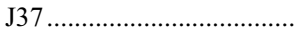 & 4895 & 2.00 & 1.45 \\
\hline C41196_2632 ...................... & 4900 & 2.00 & 1.5 \\
\hline 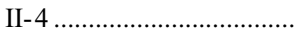 & 4910 & 2.05 & 1.5 \\
\hline IV-29 …… & 5007 & 2.22 & 1.55 \\
\hline $\mathrm{J} 45$ & 5055 & 2.25 & 1.55 \\
\hline C41155_3103 ..................... & 5068 & 2.37 & 1.55 \\
\hline I-5 & 5070 & 2.45 & 1.5 \\
\hline C41148_3103_...................... & 5247 & 2.89 & 1.55 \\
\hline C40559_2839..................... & 5349 & 3.10 & 1.6 \\
\hline C41134_3056_................... & 5305 & 3.25 & 1.0 \\
\hline C41099_3046_..................... & 5414 & 3.35 & 1.05 \\
\hline C40535_2813 ........................ & 5718 & 3.60 & 2.3 \\
\hline C41101_3050_................... & 5370 & 3.30 & 1.2 \\
\hline C41135_3053_....................... & 5500 & 3.50 & 1.15 \\
\hline C41133_2750 ............... & 5520 & 3.50 & 1.65 \\
\hline C40535_2819_................. & 5722 & 3.65 & 2.05 \\
\hline C41135_2753 .................... & 6045 & 3.80 & 2.3 \\
\hline
\end{tabular}

${ }^{\text {a }}$ Identifications as in notes to Table 1B.

erous $3 \%$ in $T_{\text {eff }}$, and ignoring any covariance, leads to a potential systematic error of \pm 0.2 dex for $\log (g)$.

\section{ABUNDANCE ANALYSIS}

We rely heavily in the present work on the procedures and atomic data for abundance analyses of metal-poor stars described in our earlier papers referenced above reporting analyses of globular cluster stars. Given the derived stellar parameters from Table 5A (Table 5B), we determined the abundances using the equivalent widths obtained as described above. The abundance analysis is carried out using a current version of the LTE spectral synthesis program MOOG (Sneden 1973). We employ the grid of stellar atmospheres from Kurucz (1993a) without convective overshoot, when available. We compute the abundances of the species observed in each star using the four stellar atmosphere models with the closest $T_{\text {eff }}$ and $\log (g)$ to each star's parameters. The abundances were interpolated using results from the closest stellar model atmospheres to the appropriate $T_{\text {eff }}$ and $\log (g)$ for each star given in Table 5A (Table 5B).

The microturbulent velocity $\left(v_{t}\right)$ of a star is determined spectroscopically by requiring the abundance to be independent of the strength of the lines. The uncertainty in our derived $v_{t}$ is estimated to be $+0.4,-0.2 \mathrm{~km} \mathrm{~s}^{-1}$ on the basis of repeated trials with the same line list for several stars varying $v_{t}$. We apply this technique here to the large sample of detected $\mathrm{Fe}_{\mathrm{I}}$ lines in each star; the results are listed with the stellar parameters in Table 5A (Table 5B).

Iron is the only element with more than a few detected lines in each of two different stages of ionization and hence is useful for determining the ionization equilibrium. Figure 2 (Fig. 3) shows the $\mathrm{Fe}$ abundance as inferred from lines of $\mathrm{Fe} \mathrm{I}$, as well as the $\mathrm{Fe}$ ionization equilibrium for the stars in our sample in 


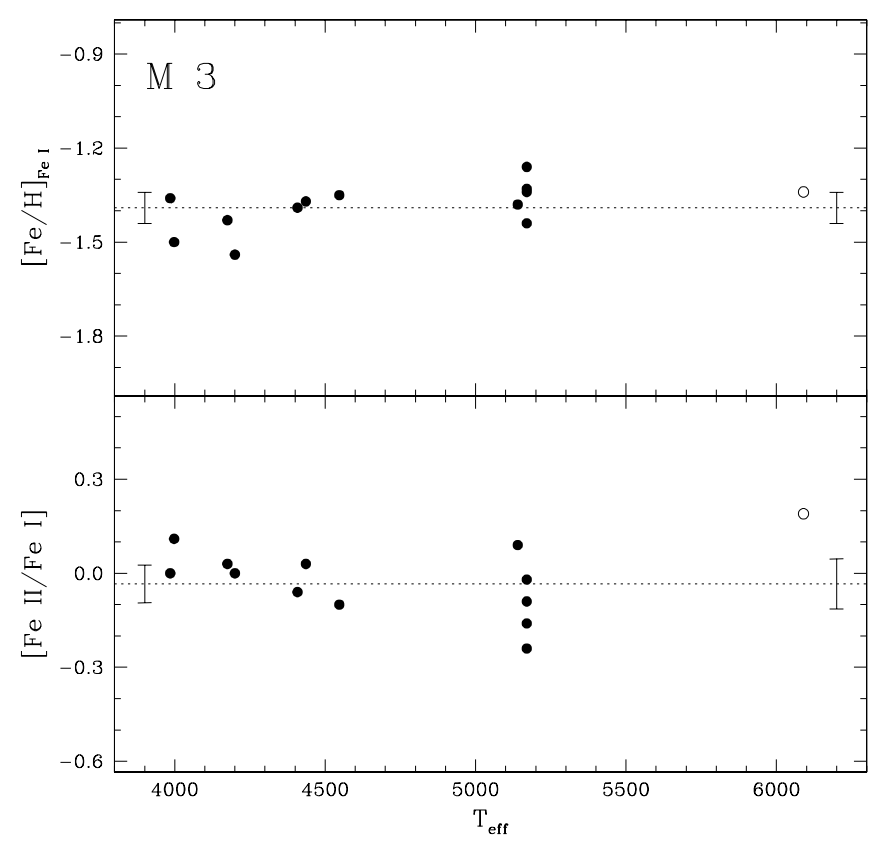

FIG. 2.- $[\mathrm{Fe} / \mathrm{H}]$ from lines of $\mathrm{Fe}$ I shown as a function of $T_{\text {eff }}$ in the top panel, while the bottom panel shows the ionization equilibrium of Fe for our sample of 13 stars in M3. The open circle indicates the HB star. The error bars on the left margin are those of the most luminous giants, while the error bars on the right margin are those of the faintest stars in our sample. The dotted horizontal line indicates the mean value for our sample in this globular cluster.

M3 (M13). The ionization equilibrium for Fe I versus Fe II is satisfactory in each of these globular clusters. The average difference between $[\mathrm{Fe} / \mathrm{H}]$ as inferred from $\mathrm{Fe}$ II lines and from Fe I lines for the eight luminous RGB stars in M3 is 0.00 dex, while the largest difference (in absolute value) is only 0.11 dex. For the five low-luminosity giants in M3, the range is somewhat larger, but the average difference is only $0.01 \mathrm{dex}$. Over the full M13 sample, the average difference is only 0.04 dex. The Fe ionization equilibrium shifts by 0.2 dex for a $100 \mathrm{~K}$ change in $T_{\text {eff }}$ in this temperature regime, so our $\pm 50 \mathrm{~K}$ uncertainty in $T_{\text {eff }}$ is capable of producing the observed dispersion in Fe ionization equilibrium. For Ti, which has far fewer detected lines of the neutral species than does $\mathrm{Fe}$, the mean difference in $[\mathrm{Ti} / \mathrm{Fe}]$, as deduced from the neutral lines and from the ionized lines for the stars in our sample in M3 (M13), is only $0.10(0.15)$ dex.

Following upon our previous work, no non-LTE corrections have been applied for the specific ions studied in the M3 and M13 stars, with the exception of O, for which we rely on the calculations of Gratton et al. (1999). The detailed non-LTE calculations of Gratton et al. (1999) and of Takeda et al. (2003) for the two $\mathrm{Na}$ I doublets we use suggest that for this regime of

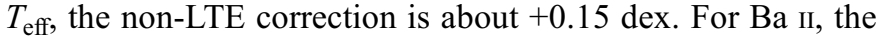
non-LTE calculations of Mashonkina \& Gehren (2000) and of Mashonkina et al. (1999) suggest that a non-LTE correction of -0.1 dex is appropriate for the metallicity of M3 and M13 and the set of $\mathrm{Ba}$ II lines we used. In comparing with other abundance analyses, the issue of implementing non-LTE corrections and their adopted values must be considered.

The resulting abundance ratios for 13 (25) stars in M3 (M13) are given in Tables $6 \mathrm{~A}, 6 \mathrm{~B}, 6 \mathrm{C}, 6 \mathrm{D}$, and $6 \mathrm{E}$ ( Tables 7A, 7B, 7C, $7 \mathrm{D}$, and $7 \mathrm{E}$ ). The abundance ratio for a species with only one detected line in a particular star is assigned an uncertainty of 0.10 dex. Table 8 indicates the changes in derived abundance ratios for small changes in the adopted stellar parameters, the $[\mathrm{Fe} / \mathrm{H}]$ for the adopted model atmosphere, or the set of $W_{\lambda}$ for

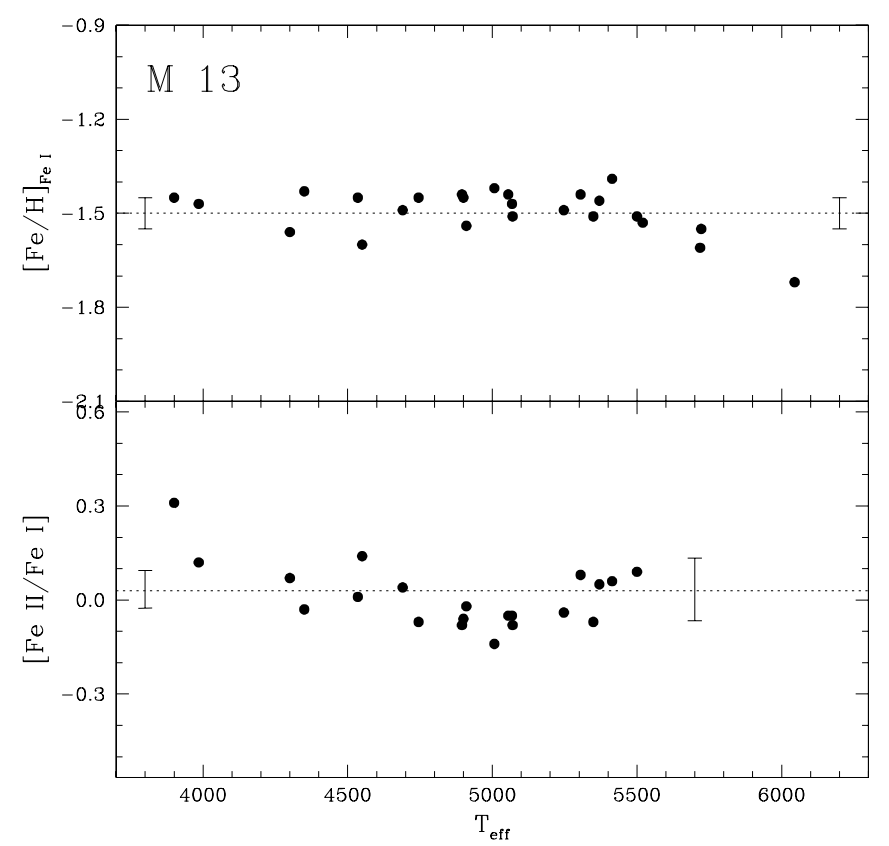

FIG. 3.- $[\mathrm{Fe} / \mathrm{H}]$ from lines of $\mathrm{Fe}$ I shown as a function of $T_{\text {eff }}$ in the top panel, while the bottom panel shows the ionization equilibrium of Fe for our sample of 25 stars in M13. The error bars on the left margin are those of the most luminous giants, while the error bars on the right margin are those of the faintest stars in our sample. The dotted horizontal line indicates the mean value for our sample in this globular cluster.

the lines of each species. Table 9 (Table 10) gives the mean abundance and $1 \sigma$ variance for the species observed in M3 (M13).

\subsection{Comments on Individual Elements}

The oxygen abundance is derived from the forbidden lines at 6300 and $6363 \AA$ and from the triplet lines at $7770 \AA$, when these were included within the wavelength range and were detected. The subtraction of the night-sky emission lines for the forbidden lines was reasonably straightforward given that the radial velocities of M3 and of M13 sufficiently different from $0 \mathrm{~km} \mathrm{~s}^{-1}$ that their $W_{\lambda}$ can be reliably measured. The $\mathrm{C} / \mathrm{O}$ ratio was assumed to be solar. Small non-LTE corrections, calculated from Gratton et al. (1999), were applied for abundances deduced from the $\mathrm{O}$ I triplet. Since $N(\mathrm{CN}) / N(\mathrm{H})$ is roughly $\propto\{N(\mathrm{Fe}) / N(\mathrm{H})\}^{2}, \mathrm{CN}$ lines are much weaker relative to $\mathrm{O}$ lines in metal-poor stars, so that the correction for $\mathrm{CN}$ contamination to the $6363 \AA\left[\mathrm{O}_{\mathrm{I}}\right]$ line for the M3 and M13 stars is negligible. Finally, the contribution of the $\mathrm{Ni}$ I line to the $6300 \AA$ forbidden line of $\mathrm{O}$ can be ignored in M3 and M13, as we will see that $\mathrm{O} / \mathrm{Fe}$ (and $\mathrm{O} / \mathrm{Ni}$ ) is larger than the solar value. ${ }^{5}$ The $\mathrm{O}$ abundance from the triplet lines is given with respect to $[\mathrm{Fe} / \mathrm{H}]$ deduced from lines of $\mathrm{Fe} \mathrm{I}$, while that from the forbidden lines is given with respect to $\mathrm{Fe}$ II; the mean $[\mathrm{O} / \mathrm{Fe}]$ becomes 0.04 dex larger if expressed using the Fe II lines instead.

The usual lines of $\mathrm{Al}$ in this wavelength region, in particular the doublet at $6696,6698 \AA$, cannot be reached with our HIRES configuration as they fall in an interorder gap. We have detected the much weaker Al I line at $5557 \AA$ in the coolest M13 giants only. There is a difference of 0.4 dex between the $\mathrm{Al}$ abundance we deduce and that obtained by Sneden et al. (2004) (see also

\footnotetext{
${ }^{5}$ Sneden et al. (2004) estimate that $W_{\lambda}$ for this Ni I line is less than $0.5 \mathrm{~m} \AA$.
} 
TABLE 6A

Abundance Ratios for M3: O to Mg

\begin{tabular}{|c|c|c|c|c|c|c|c|c|c|c|}
\hline Star & $\begin{array}{c}{[\mathrm{Fe} / \mathrm{H}]_{\mathrm{I}} \pm \sigma / \sqrt{N}} \\
(\mathrm{dex})\end{array}$ & $N$ & $\begin{array}{c}{[\mathrm{Fe} / \mathrm{H}]_{\mathrm{II}} \pm \sigma / \sqrt{N}} \\
(\mathrm{dex})\end{array}$ & $N$ & $\begin{array}{c}{[\mathrm{O} / \mathrm{Fe}] \pm \sigma / \sqrt{N}} \\
(\operatorname{dex})\end{array}$ & $N$ & $\begin{array}{c}{[\mathrm{Na} / \mathrm{Fe}] \pm \sigma / \sqrt{N}} \\
(\mathrm{dex})\end{array}$ & $N$ & $\begin{array}{c}{[\mathrm{Mg} / \mathrm{Fe}] \pm \sigma / \sqrt{N}} \\
(\mathrm{dex})\end{array}$ & $N$ \\
\hline 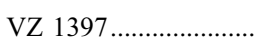 & $-1.36 \pm 0.05^{\mathrm{a}}$ & 149 & $-1.36 \pm 0.06$ & 13 & $0.52 \pm 0.05^{\mathrm{a}}$ & 2 & $-0.16 \pm 0.05$ & 4 & $0.59 \pm 0.13$ & 3 \\
\hline 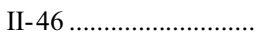 & $-1.50 \pm 0.05^{\mathrm{a}}$ & 148 & $-1.39 \pm 0.05$ & 13 & $0.37 \pm 0.05^{\mathrm{a}}$ & 2 & $-0.23 \pm 0.07$ & 4 & $0.56 \pm 0.15$ & 3 \\
\hline VZ $1000 \ldots \ldots \ldots \ldots$ & $-1.43 \pm 0.05^{\mathrm{a}}$ & 143 & $-1.40 \pm 0.05$ & 13 & $0.08 \pm 0.10$ & 2 & $+0.10 \pm 0.06$ & 4 & $0.44 \pm 0.10$ & 3 \\
\hline III-28 & $-1.54 \pm 0.05^{\mathrm{a}}$ & 134 & $-1.54 \pm 0.05$ & 10 & $0.55 \pm 0.07$ & 2 & $-0.33 \pm 0.06$ & 2 & $0.49 \pm 0.15$ & 3 \\
\hline IV-25 & $-1.39 \pm 0.05^{\mathrm{a}}$ & 141 & $-1.45 \pm 0.05^{\mathrm{a}}$ & 14 & $0.38 \pm 0.05^{\mathrm{a}}$ & 2 & $-0.10 \pm 0.05$ & 4 & $0.41 \pm 0.11$ & 3 \\
\hline C41303_2217 .............. & $-1.37 \pm 0.05^{\mathrm{a}}$ & 126 & $-1.34 \pm 0.05^{\mathrm{a}}$ & 14 & $0.33 \pm 0.05^{\mathrm{a}}$ & 2 & $-0.39 \pm 0.09$ & 3 & $0.40 \pm 0.11$ & 3 \\
\hline IV-27 .......................... & $-1.35 \pm 0.05^{\mathrm{a}}$ & 129 & $-1.45 \pm 0.05$ & 10 & $0.08 \pm 0.08$ & 2 & $+0.11 \pm 0.06$ & 4 & $0.31 \pm 0.17$ & 3 \\
\hline 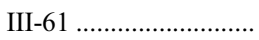 & $-1.38 \pm 0.05^{\mathrm{a}}$ & 61 & $-1.29 \pm 0.07$ & 3 & $0.60 \pm 0.05$ & 2 & $-0.45 \pm 0.09$ & 2 & $0.29 \pm 0.10$ & 1 \\
\hline 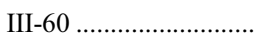 & $-1.33 \pm 0.05^{\mathrm{a}}$ & 65 & $-1.49 \pm 0.10$ & 3 & $0.29 \pm 0.05^{\mathrm{a}}$ & 2 & $-0.23 \pm 0.13$ & 2 & $0.25 \pm 0.10$ & 1 \\
\hline C41544_2336.............. & $-1.26 \pm 0.05^{\mathrm{a}}$ & 85 & $-1.50 \pm 0.07$ & 8 & $0.39 \pm 0.05^{\mathrm{a}}$ & 2 & $-0.20 \pm 0.05^{\mathrm{a}}$ & 2 & $0.48 \pm 0.09$ & 2 \\
\hline 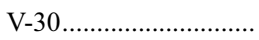 & $-1.34 \pm 0.05^{\mathrm{a}}$ & 80 & $-1.43 \pm 0.06$ & 8 & $\leq-0.03 \pm 0.10$ & 1 & $+0.16 \pm 0.05^{\mathrm{a}}$ & 2 & $0.35 \pm 0.22$ & 2 \\
\hline V-31 ............................... & $-1.44 \pm 0.05^{\mathrm{a}}$ & 76 & $-1.46 \pm 0.04^{\mathrm{a}}$ & 8 & $0.35 \pm 0.10$ & 1 & $-0.20 \pm 0.05^{\mathrm{a}}$ & 2 & $0.22 \pm 0.11$ & 2 \\
\hline C41543_2334_............. & $-1.34 \pm 0.05^{\mathrm{a}}$ & 43 & $-1.15 \pm 0.06$ & 8 & $0.50 \pm 0.07$ & 3 & $+0.25 \pm 0.05^{\mathrm{a}}$ & 2 & $0.51 \pm 0.08$ & 2 \\
\hline
\end{tabular}

a The minimum value of 0.05 dex has been adopted; the nominal calculated value is smaller.

Kraft et al. 1997; Shetrone 1996) for the two M13 luminous giants in common. The $g f$-value adopted for this rarely used line ( -1.67 dex) may be wrong, or non-LTE may play a role; Baumüller \& Gehren (1997) have demonstrated that non-LTE effects can be very strong for $\mathrm{Al}$ I features in this $T_{\text {eff }}$ range, and the effect varies a lot from multiplet to multiplet.

The Na abundance was obtained from the doublet at $5680 \AA$ in general. For the faintest, hottest stars in M13, these lines become very weak, and so the $\mathrm{Na}$ abundance was checked using the $\mathrm{D}$ lines (after a small empirical correction not exceeding 0.08 dex to put them on the same abundance scale as for the weak $\mathrm{Na}$ lines).

The abundances of the elements with respect to $\mathrm{Fe},[\mathrm{X} / \mathrm{Fe}]$, as a function of $T_{\text {eff }}$ are shown in Figure 4 (Fig. 5), covering O, Na, $\mathrm{Mg}$, and $\mathrm{Si}$; Figure 6 (Fig. 7), which includes $\mathrm{Ca}, \mathrm{Sc}$, Ti and V; Figure 8 (Fig. 9), which includes $\mathrm{Cr}, \mathrm{Mn}, \mathrm{Co}$, and $\mathrm{Ni}$; Figure 10 (Fig. 11), which includes $\mathrm{Cu}, \mathrm{Zn}, \mathrm{Y}$, and $\mathrm{Zr}$; and Figure 12 (Fig. 13) for $\mathrm{Ba}, \mathrm{La}, \mathrm{Nd}, \mathrm{Eu}$, and Dy. Note the apparent star-tostar variation in $[\mathrm{O} / \mathrm{Fe}]$ and in $[\mathrm{Na} / \mathrm{Fe}]$, which becomes undetectably small, if it exists at all, for the elements heavier than $\mathrm{Na}$. The scatter for Ni, which is detected with several lines in every star, is remarkably small. Even the rare earths, for which in general only a few weak lines are detected only in the more luminous stars, show very small variations in general.

\subsection{Abundance Spreads}

Detection and quantitative measurement of star-to-star variations in abundance ratios within a single Galactic globular cluster is one of the primary goals of this effort. As a global indicator of the presence of such effects we use a parameter we call the "spread ratio" (SR). The numerator of SR is the $1 \sigma \mathrm{rms}$ variance for the sample of 13 (25) stars in M3 (M13) about that mean abundance for each atomic species (X) with detected absorption lines, denoted $\sigma$. The mean abundance and $\sigma$ for each species observed are given in the first three columns of Table 9 (Table 10). The denominator of SR is the total expected uncertainty, $\sigma($ tot $)$, which is the sum in quadrature of the known contributing terms. Included are a term corresponding to an uncertainty of $50 \mathrm{~K}$ in $T_{\text {eff }}$, the same for an uncertainty of 0.2 dex in $\log (g)$, as well as for an uncertainty of $0.2 \mathrm{~km} \mathrm{~s}^{-1}$ in $v_{t}$ and the observed uncertainty $[\sigma(\mathrm{obs})]$. The parameter $\sigma(\mathrm{obs})$, which is calculated from data given in Tables $6 \mathrm{~A}, 6 \mathrm{~B}, 6 \mathrm{C}, 6 \mathrm{D}$, and $6 \mathrm{E}$ (Tables 7A7B, 7C, 7D, and 7E), is taken as the variance about the mean abundance for a given species in a given star, i.e., the $1 \sigma \mathrm{rms}$ value about the mean abundance of species $\mathrm{X}$ in a given star $/ \sqrt{N}$, where $N$ is the number of observed lines of species $\mathrm{X}$. It includes contributions from errors in the measured $W_{\lambda}$,

TABLE 6B

Abundance Ratios for M3: Si to V

\begin{tabular}{|c|c|c|c|c|c|c|c|c|c|c|}
\hline Star & $\begin{array}{c}{[\mathrm{Si} / \mathrm{Fe}] \pm \sigma / \sqrt{N}} \\
(\operatorname{dex})\end{array}$ & $N$ & $\begin{array}{c}{[\mathrm{Ca} / \mathrm{Fe}] \pm \sigma / \sqrt{N}} \\
(\operatorname{dex})\end{array}$ & $N$ & $\begin{array}{c}{[\mathrm{Sc} / \mathrm{Fe}] \pm \sigma / \sqrt{N}} \\
(\operatorname{dex})\end{array}$ & $N$ & $\begin{array}{c}{[\mathrm{Ti} / \mathrm{Fe}] \pm \sigma / \sqrt{N}} \\
(\mathrm{dex})\end{array}$ & $N$ & $\begin{array}{c}{[\mathrm{V} / \mathrm{Fe}] \pm \sigma / \sqrt{N}} \\
(\mathrm{dex})\end{array}$ & $N$ \\
\hline VZ $1397 \ldots \ldots \ldots \ldots . .$. & $0.35 \pm 0.05^{\mathrm{a}}$ & 11 & $0.09 \pm 0.05^{\mathrm{a}}$ & 16 & $0.10 \pm 0.06$ & 7 & $0.30 \pm 0.05^{\mathrm{a}}$ & 35 & $0.05 \pm 0.06$ & 9 \\
\hline 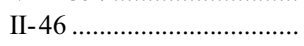 & $0.28 \pm 0.06$ & 9 & $-0.02 \pm 0.05^{\mathrm{a}}$ & 15 & $0.13 \pm 0.05^{\mathrm{a}}$ & 7 & $0.08 \pm 0.05^{\mathrm{a}}$ & 33 & $-0.21 \pm 0.05$ & 9 \\
\hline 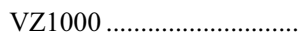 & $0.35 \pm 0.05^{\mathrm{a}}$ & 9 & $0.11 \pm 0.05^{\mathrm{a}}$ & 15 & $0.19 \pm 0.05$ & 7 & $0.13 \pm 0.05^{\mathrm{a}}$ & 32 & $-0.10 \pm 0.05^{\mathrm{a}}$ & 10 \\
\hline 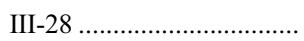 & $0.19 \pm 0.08$ & 3 & $0.05 \pm 0.05^{\mathrm{a}}$ & 15 & $0.15 \pm 0.07$ & 7 & $0.11 \pm 0.05^{\mathrm{a}}$ & 29 & $-0.20 \pm 0.05^{\mathrm{a}}$ & 10 \\
\hline 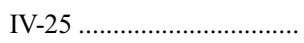 & $0.25 \pm 0.05^{\mathrm{a}}$ & 9 & $0.12 \pm 0.05^{\mathrm{a}}$ & 15 & $0.11 \pm 0.05$ & 7 & $0.10 \pm 0.05^{\mathrm{a}}$ & 26 & $-0.17 \pm 0.05^{\mathrm{a}}$ & 8 \\
\hline C41303_2217 ...................... & $0.20 \pm 0.05^{\mathrm{a}}$ & 9 & $0.08 \pm 0.05^{\mathrm{a}}$ & 15 & $0.15 \pm 0.05$ & 7 & $0.10 \pm 0.05^{\mathrm{a}}$ & 25 & $-0.18 \pm 0.06$ & 8 \\
\hline 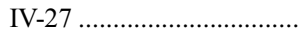 & $0.23 \pm 0.05^{\mathrm{a}}$ & 12 & $0.10 \pm 0.05^{\mathrm{a}}$ & 15 & $0.18 \pm 0.06$ & 7 & $0.10 \pm 0.05^{\mathrm{a}}$ & 27 & $-0.15 \pm 0.05^{\mathrm{a}}$ & 8 \\
\hline III-61 ………… & $0.23 \pm 0.13$ & 4 & $0.18 \pm 0.05$ & 17 & $0.21 \pm 0.11$ & 7 & $0.13 \pm 0.06$ & 3 & $\ldots$ & 0 \\
\hline 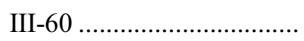 & $0.29 \pm 0.05^{\mathrm{a}}$ & 5 & $0.14 \pm 0.05^{\mathrm{a}}$ & 17 & $0.08 \pm 0.08$ & 7 & $0.26 \pm 0.06$ & 5 & $\ldots$ & 0 \\
\hline C41544_2336 ................ & $0.32 \pm 0.06$ & 8 & $0.12 \pm 0.05^{\mathrm{a}}$ & 17 & $0.04 \pm 0.05$ & 7 & $0.27 \pm 0.06$ & 13 & $\ldots$ & 0 \\
\hline V-30 & $0.19 \pm 0.05$ & 5 & $0.05 \pm 0.05^{\mathrm{a}}$ & 17 & $0.13 \pm 0.06$ & 7 & $0.21 \pm 0.05$ & 11 & $\ldots$ & 0 \\
\hline 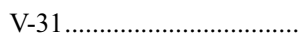 & $0.30 \pm 0.06$ & 4 & $0.12 \pm 0.05^{\mathrm{a}}$ & 16 & $0.10 \pm 0.05$ & 7 & $0.19 \pm 0.05^{\mathrm{a}}$ & 10 & $\ldots$ & 0 \\
\hline C41543_2334...................... & $0.32 \pm 0.05^{\mathrm{a}}$ & 2 & $0.22 \pm 0.05$ & 9 & $-0.07 \pm 0.25$ & 2 & $0.18 \pm 0.05^{\mathrm{a}}$ & 3 & $\ldots$ & 0 \\
\hline
\end{tabular}

a The minimum value of 0.05 dex has been adopted; the nominal calculated value is smaller. 
TABLE 6C

Abundance Ratios for M3: $\mathrm{Cr}$ to $\mathrm{Cu}$

\begin{tabular}{|c|c|c|c|c|c|c|c|c|c|c|}
\hline Star & $\begin{array}{c}{[\mathrm{Cr} / \mathrm{Fe}] \pm \sigma / \sqrt{N}} \\
(\mathrm{dex})\end{array}$ & $N$ & $\begin{array}{c}{[\mathrm{Mn} / \mathrm{Fe}] \pm \sigma / \sqrt{N}} \\
(\mathrm{dex})\end{array}$ & $N$ & $\begin{array}{c}{[\mathrm{Co} / \mathrm{Fe}] \pm \sigma / \sqrt{N}} \\
(\mathrm{dex})\end{array}$ & $N$ & $\begin{array}{c}{[\mathrm{Ni} / \mathrm{Fe}] \pm \sigma / \sqrt{N}} \\
(\mathrm{dex})\end{array}$ & $N$ & $\begin{array}{c}{[\mathrm{Cu} / \mathrm{Fe}] \pm \sigma / \sqrt{N}} \\
(\mathrm{dex})\end{array}$ & $N$ \\
\hline 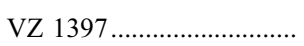 & $0.03 \pm 0.06$ & 8 & $-0.11 \pm 0.14$ & 5 & $-0.01 \pm 0.05$ & 4 & $-0.10 \pm 0.05^{\mathrm{a}}$ & 24 & $-0.55 \pm 0.09$ & 3 \\
\hline II-46 & $-0.06 \pm 0.05$ & 8 & $-0.20 \pm 0.15$ & 5 & $-0.08 \pm 0.06$ & 4 & $-0.13 \pm 0.05^{\mathrm{a}}$ & 21 & $-0.55 \pm 0.09$ & 3 \\
\hline VZ $1000 \ldots \ldots \ldots \ldots \ldots$ & $-0.10 \pm 0.09$ & 9 & $-0.25 \pm 0.09$ & 5 & $-0.07 \pm 0.09$ & 4 & $-0.14 \pm 0.05^{\mathrm{a}}$ & 20 & $-0.51 \pm 0.06$ & 3 \\
\hline III-28 & $0.10 \pm 0.08$ & 8 & $-0.34 \pm 0.12$ & 3 & $-0.02 \pm 0.09$ & 4 & $-0.18 \pm 0.05^{\mathrm{a}}$ & 13 & $-0.66 \pm 0.05^{\mathrm{a}}$ & 3 \\
\hline IV-25 ............................. & $0.05 \pm 0.06$ & 10 & $-0.30 \pm 0.12$ & 5 & $-0.06 \pm 0.08$ & 4 & $-0.14 \pm 0.05^{\mathrm{a}}$ & 20 & $-0.62 \pm 0.11$ & 2 \\
\hline C41303_2217 ……........ & $0.01 \pm 0.06$ & 9 & $-0.27 \pm 0.14$ & 4 & $-0.11 \pm 0.03$ & 3 & $-0.16 \pm 0.05^{\mathrm{a}}$ & 15 & $-0.56 \pm 0.21$ & 2 \\
\hline 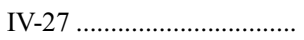 & $-0.03 \pm 0.05^{\mathrm{a}}$ & 9 & $-0.17 \pm 0.05$ & 3 & $-0.16 \pm 0.06$ & 3 & $-0.16 \pm 0.05^{\mathrm{a}}$ & 14 & $-0.71 \pm 0.05^{\mathrm{a}}$ & 2 \\
\hline 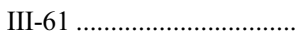 & $\ldots$ & 0 & $\ldots$ & 0 & $\ldots$ & 0 & $-0.32 \pm 0.08$ & 6 & $\ldots$ & 0 \\
\hline 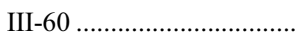 & $\ldots$ & 0 & $\ldots$ & 0 & $\ldots$ & 0 & $-0.24 \pm 0.10$ & 6 & $\ldots$ & 0 \\
\hline C41544_2336................... & $-0.32 \pm 0.09$ & 4 & $-0.32 \pm 0.17$ & 3 & $\ldots$ & 0 & $-0.08 \pm 0.05$ & 4 & $-0.46 \pm 0.10$ & 1 \\
\hline 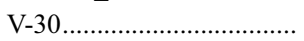 & $-0.26 \pm 0.07$ & 3 & $-0.44 \pm 0.05$ & 3 & $\ldots$ & 0 & $-0.17 \pm 0.09$ & 4 & $-0.56 \pm 0.10$ & 1 \\
\hline V-31 & $-0.17 \pm 0.12$ & 4 & $-0.33 \pm 0.09$ & 3 & $\ldots$ & 0 & $-0.13 \pm 0.05^{\mathrm{a}}$ & 6 & $-0.57 \pm 0.10$ & 1 \\
\hline C41543_2334..................... & $-0.07 \pm 0.17$ & 3 & $-0.43 \pm 0.06$ & 3 & $\ldots$ & 0 & $\ldots$ & 0 & & 0 \\
\hline
\end{tabular}

a The minimum value of 0.05 dex has been adopted; the nominal calculated value is smaller.

random errors (i.e., between lines of a given species) in the adopted $g f$-values, etc. Some species, an example being Fe I with its very large value of $N$, have unrealistically small values of $\sigma(\mathrm{obs})$; we adopt a minimum of 0.05 dex for this parameter.

The ratio $\sigma / \sigma$ (tot) is an indication of whether there is any intrinsic star-to-star variation in $[\mathrm{X} / \mathrm{Fe}]$. A high value of this SRspread ratio, tabulated in the fifth column of this table, suggests a high probability of intrinsic scatter for the abundance of the species X. Ideally, the mean SR for those elements with no star-to-star variation should be unity. However, we use the entries in Table 8 for the lowest $T_{\text {eff }}$, i.e., for giants near the RGB tip, to calculate $\sigma$ (tot). Since the abundance sensitivities in general decrease as $T_{\text {eff }}$ increases, we will slightly overestimate $\sigma$ (tot) and hence underestimate SR, thus explaining why the deduced values for SR tend to be slightly less than 1. A second indication of the reality of the star-to-star variation in $[\mathrm{X} / \mathrm{Fe}]$ for any species $\mathrm{X}$ is deduced using a $\chi^{2}$ analysis and evaluating the probability of exceeding by chance the measured value of $\chi^{2}$ from our sample of stars in a globular cluster. Only $\mathrm{O}$ I and $\mathrm{Na}$ I have values of $\chi^{2}$ much higher than the number of degrees of freedom [N(star) - 1], where $N(\operatorname{star})$ is the number of stars in which that species was detected.

Inspection of Table 9 shows that for all but two species the SR in M3 ranges from 0.5 to 1.1 , indicating little sign of an intrinsic star-to-star range in abundance. $\mathrm{O}$ I and $\mathrm{Na} \mathrm{I}$, however, have SR exceeding 2.0, and their $\chi^{2}$-values are very large. Note that $\mathrm{SR}$ for $\mathrm{Mg}$ I is 0.7 , suggesting no real star-to-star abundance variations for this element in M3. For M13, $\mathrm{O}$ I, and $\mathrm{Na}$ I again have by far the largest values of SR, exceeding 2.5 in both cases, with no other species having a value exceeding 1.5 . We therefore assume that the range of abundances seen in our M3 and in our M13 samples for $\mathrm{Na}$ I and $\mathrm{O}$ I represent real star-tostar abundance variations; while no other element shows definite evidence for such variations from this simple analysis (but see $\S 7$ ).

We have also examined whether one can discern a difference in the mean $\langle[\mathrm{Fe} / \mathrm{H}]\rangle$ between stars with high and low $\mathrm{O}$ abundances in M13 and between stars with high and low Na abundances in M13. No statistically significant difference was found.

\subsection{The Peculiar Star M13 I-5}

With regard to the heavy elements, astute readers will have noticed that there is one star (M13 I-5) with anomalously high Y and $\mathrm{Ba}$ (see Table 7D). This star, which is too faint to have been included in any previous high-dispersion analyses in this globular cluster, stands out (and is marked) in Figure 11 (Y panel) and in Figure 13 (Ba panel). It has the highest abundance of each

TABLE 6D

Abundance Ratios for M3: $\mathrm{Zn}$ to La

\begin{tabular}{|c|c|c|c|c|c|c|c|c|c|c|}
\hline Star & $\begin{array}{c}{[\mathrm{Zn} / \mathrm{Fe}] \pm \sigma / \sqrt{N}} \\
(\operatorname{dex})\end{array}$ & $N$ & $\begin{array}{c}{[\mathrm{Y} / \mathrm{Fe}] \pm \sigma / \sqrt{N}} \\
(\operatorname{dex})\end{array}$ & $N$ & $\begin{array}{c}{[\mathrm{Zr} / \mathrm{Fe}] \pm \sigma / \sqrt{N}} \\
(\operatorname{dex})\end{array}$ & $N$ & $\begin{array}{c}{[\mathrm{Ba} / \mathrm{Fe}] \pm \sigma / \sqrt{N}} \\
(\mathrm{dex})\end{array}$ & $N$ & $\begin{array}{c}{[\mathrm{La} / \mathrm{Fe}] \pm \sigma / \sqrt{N}} \\
(\mathrm{dex})\end{array}$ & $N$ \\
\hline VZ $1397 \ldots$ & $-0.14 \pm 0.05^{\mathrm{a}}$ & 2 & $-0.56 \pm 0.07$ & 3 & $0.13 \pm 0.05$ & 3 & $0.13 \pm 0.05$ & 3 & $0.07 \pm 0.05$ & 3 \\
\hline 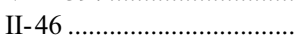 & $0.09 \pm 0.08$ & 2 & $-0.27 \pm 0.10$ & 3 & $-0.12 \pm 0.05^{\mathrm{a}}$ & 3 & $0.12 \pm 0.05$ & 3 & $0.03 \pm 0.08$ & 3 \\
\hline 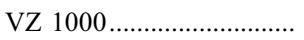 & $-0.12 \pm 0.05^{\mathrm{a}}$ & 2 & $-0.25 \pm 0.09$ & 4 & $-0.11 \pm 0.05^{\mathrm{a}}$ & 3 & $0.15 \pm 0.06$ & 3 & $0.01 \pm 0.05$ & 3 \\
\hline 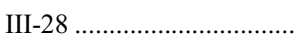 & $-0.12 \pm 0.05^{\mathrm{a}}$ & 2 & $-0.14 \pm 0.13$ & 4 & $-0.03 \pm 0.06$ & 3 & $0.08 \pm 0.09$ & 3 & $0.17 \pm 0.08$ & 2 \\
\hline IV-25 & $-0.10 \pm 0.07$ & 2 & $-0.25 \pm 0.09$ & 4 & $0.05 \pm 0.05$ & 3 & $0.15 \pm 0.05^{\mathrm{a}}$ & 3 & $-0.02 \pm 0.05$ & 3 \\
\hline C41303_2217 ..................... & $-0.10 \pm 0.05^{\mathrm{a}}$ & 2 & $-0.15 \pm 0.10$ & 4 & $0.22 \pm 0.10$ & 3 & $0.16 \pm 0.05$ & 3 & $-0.02 \pm 0.15$ & 2 \\
\hline IV-27 …… & $-0.13 \pm 0.05^{\mathrm{a}}$ & 2 & $-0.35 \pm 0.09$ & 4 & $\ldots$ & 0 & $0.14 \pm 0.06$ & 3 & $0.05 \pm 0.05$ & 3 \\
\hline III-61 …………… & $\ldots$ & 0 & $\ldots$ & 0 & $\ldots$ & 0 & $-0.09 \pm 0.05^{\mathrm{a}}$ & 3 & $\ldots$ & 0 \\
\hline 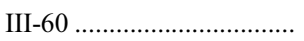 & & 0 & $\ldots$ & 0 & $\ldots$ & 0 & $0.12 \pm 0.05^{\mathrm{a}}$ & 3 & $\ldots$ & 0 \\
\hline 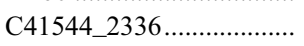 & $-0.19 \pm 0.05^{\mathrm{a}}$ & 2 & $-0.29 \pm 0.06$ & 3 & $\ldots$ & 0 & $0.24 \pm 0.09$ & 3 & $\ldots$ & 0 \\
\hline V-30 & $-0.10 \pm 0.28$ & 2 & $-0.38 \pm 0.20$ & 3 & $\ldots$ & 0 & $0.34 \pm 0.10$ & 3 & $\ldots$ & 0 \\
\hline V-31 & $0.02 \pm 0.18$ & 2 & $-0.26 \pm 0.09$ & 3 & $\ldots$ & 0 & $0.20 \pm 0.08$ & 3 & $\ldots$ & 0 \\
\hline C41543_2334_................... & $-0.15 \pm 0.05^{\mathrm{a}}$ & 2 & $-0.12 \pm 0.05^{\mathrm{a}}$ & 2 & $\ldots$ & 0 & $0.38 \pm 0.15$ & 3 & $\ldots$ & 0 \\
\hline
\end{tabular}

\footnotetext{
${ }^{\text {a }}$ The minimum value of 0.05 dex has been adopted; the nominal calculated value is smaller.
} 
TABLE 6E

Abundance Ratios for M3: Nd to Dy

\begin{tabular}{|c|c|c|c|c|c|c|}
\hline Star & $\begin{array}{c}{[\mathrm{Nd} / \mathrm{Fe}] \pm \sigma / \sqrt{N}} \\
(\mathrm{dex})\end{array}$ & $N$ & $\begin{array}{c}{[\mathrm{Eu} / \mathrm{Fe}] \pm \sigma / \sqrt{N}} \\
(\mathrm{dex})\end{array}$ & $N$ & $\begin{array}{c}{[\mathrm{Dy} / \mathrm{Fe}] \pm \sigma / \sqrt{N}} \\
\quad(\mathrm{dex})\end{array}$ & $N$ \\
\hline 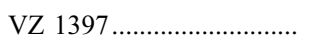 & $0.12 \pm 0.07$ & 5 & $0.46 \pm 0.10$ & 1 & $-0.04 \pm 0.10$ & 1 \\
\hline II-46 & $0.16 \pm 0.05$ & 5 & $0.51 \pm 0.10$ & 1 & $0.12 \pm 0.10$ & 1 \\
\hline VZ $1000 \ldots \ldots \ldots \ldots \ldots \ldots \ldots \ldots \ldots$ & $0.29 \pm 0.05$ & 7 & $0.44 \pm 0.10$ & 1 & $-0.09 \pm 0.10$ & 1 \\
\hline 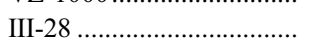 & $0.15 \pm 0.05^{\mathrm{a}}$ & 7 & $0.52 \pm 0.10$ & 1 & $0.08 \pm 0.10$ & 1 \\
\hline IV-25 & $0.16 \pm 0.05^{\mathrm{a}}$ & 7 & $0.46 \pm 0.10$ & 1 & $0.19 \pm 0.10$ & 1 \\
\hline Stet 189 & $0.28 \pm 0.06$ & 5 & $0.51 \pm 0.10$ & 1 & $\ldots$ & 0 \\
\hline 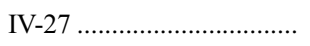 & $0.21 \pm 0.05^{\mathrm{a}}$ & 5 & $0.55 \pm 0.10$ & 1 & $\ldots$ & 0 \\
\hline 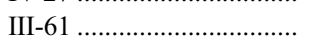 & $\ldots$ & 0 & $\ldots$ & 0 & $\ldots$ & 0 \\
\hline 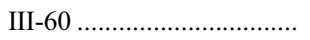 & $\ldots$ & 0 & $\ldots$ & 0 & $\ldots$ & 0 \\
\hline C41544_2336 ..................... & $-0.06 \pm 0.10$ & 1 & $0.53 \pm 0.10$ & 1 & $\ldots$ & 0 \\
\hline 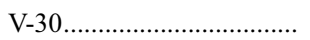 & $\ldots$ & 0 & $\ldots$ & 0 & $\ldots$ & 0 \\
\hline 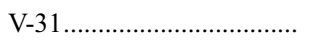 & $\ldots$ & 0 & $\ldots$ & 0 & $\ldots$ & 0 \\
\hline C41543_2334_..................... & $\ldots$ & 0 & $\ldots$ & 0 & $\ldots$ & 0 \\
\hline
\end{tabular}

a The minimum value of 0.05 dex has been adopted; the nominal calculated value is smaller.

of these two elements in the entire M13 sample; its deduced $\epsilon(\mathrm{Y})$ is 5 times the mean of the remaining 24 stars in the M13 sample. $\mathrm{Y}_{\text {II }}$ has the fourth largest values of SR $(\mathrm{SR}=1.27)$ in M13, but eliminating this star would reduce it to below 0.5 . Figure 14 shows a section of the spectrum of this star compared with one of similar luminosity and $T_{\text {eff }}$ in M13. This figure demonstrates convincingly that the very high abundance of $\mathrm{Y}$ measured for star M13 I-5 is definitely real.

With regard to the other heavy elements, in the spectrum of star M13 I-5 there is one weak and uncertain detection for La II and several weak lines ascribed to $\mathrm{Nd}$ II; these give $[\mathrm{La} / \mathrm{Fe}]$ at the upper end of those for the other M13 stars and the largest (but only by $\sim 0.05$ dex) $[\mathrm{Nd} / \mathrm{Fe}]$ for any star in our sample in M13. An upper limit to the $6645 \AA$ line of Eu II of $10.5 \mathrm{~m} \AA$ yields an upper limit to $[\mathrm{Eu} / \mathrm{Fe}]$ of +0.7 dex. Since the M13 mean for $[\mathrm{Eu} / \mathrm{Fe}]$ is $+0.57 \mathrm{dex}$, Eu is not significantly enhanced in this star, consistent with an $s$-process enhancement. A better spectrum for this peculiar star has just been acquired, and results will be reported in a future publication.

\subsection{Comparison with Previous Analyses}

Since M3 and M13 are key globular clusters, there have been several previous high-dispersion analyses of the most luminous stars in them. A comparison of the $T_{\text {eff }}$ determined in the present

TABLE 7A

Abundance Ratios for M13: O to Mg

\begin{tabular}{|c|c|c|c|c|c|c|c|c|c|c|}
\hline Star & $\begin{array}{c}{[\mathrm{Fe} / \mathrm{H}]_{\mathrm{I}} \pm \sigma / \sqrt{N}} \\
(\operatorname{dex})\end{array}$ & $N$ & $\begin{array}{c}{[\mathrm{Fe} / \mathrm{H}]_{\mathrm{II}} \pm \sigma / \sqrt{N}} \\
(\operatorname{dex})\end{array}$ & $N$ & $\begin{array}{c}{[\mathrm{O} / \mathrm{Fe}] \pm \sigma / \sqrt{N}} \\
(\operatorname{dex})\end{array}$ & $N$ & $\begin{array}{l}{[\mathrm{Na} / \mathrm{Fe}] \pm \sigma / \sqrt{N}} \\
(\mathrm{dex})\end{array}$ & $N$ & $\begin{array}{c}{[\mathrm{Mg} / \mathrm{Fe}] \pm \sigma / \sqrt{N}} \\
(\operatorname{dex})\end{array}$ & $N$ \\
\hline II-67... & $-1.45 \pm 0.05^{\mathrm{a}}$ & 110 & $-1.14 \pm 0.06$ & 12 & $-1.14 \pm 0.10$ & 1 & $0.32 \pm 0.05$ & 3 & $0.29 \pm 0.19$ & 3 \\
\hline IV-25 & $-1.47 \pm 0.05^{\mathrm{a}}$ & 128 & $-1.35 \pm 0.05$ & 12 & $-0.33 \pm 0.05^{\mathrm{a}}$ & 2 & $0.41 \pm 0.09$ & 4 & $0.35 \pm 0.21$ & 3 \\
\hline 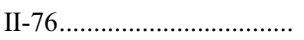 & $-1.56 \pm 0.05^{\mathrm{a}}$ & 123 & $-1.49 \pm 0.05^{\mathrm{a}}$ & 13 & $0.55 \pm 0.05^{\mathrm{a}}$ & 2 & $-0.32 \pm 0.05$ & 4 & $0.49 \pm 0.17$ & 3 \\
\hline 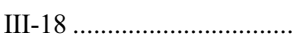 & $-1.43 \pm 0.05^{\mathrm{a}}$ & 123 & $-1.46 \pm 0.05^{\mathrm{a}}$ & 13 & $-0.35 \pm 0.10$ & 1 & $0.36 \pm 0.06$ & 4 & $0.29 \pm 0.14$ & 3 \\
\hline 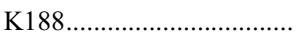 & $-1.45 \pm 0.05^{\mathrm{a}}$ & 111 & $-1.44 \pm 0.05^{\mathrm{a}}$ & 13 & $0.33 \pm 0.07$ & 2 & $-0.08 \pm 0.05^{\mathrm{a}}$ & 4 & $0.39 \pm 0.17$ & 3 \\
\hline III-7 ……… & $-1.60 \pm 0.05^{\mathrm{a}}$ & 93 & $-1.46 \pm 0.05^{\mathrm{a}}$ & 13 & $0.46 \pm 0.08$ & 2 & $0.10 \pm 0.05^{\mathrm{a}}$ & 2 & $0.52 \pm 0.14$ & 3 \\
\hline I-18 & $-1.49 \pm 0.05^{\mathrm{a}}$ & 88 & $-1.45 \pm 0.05^{\mathrm{a}}$ & 13 & $0.17 \pm 0.10$ & 1 & $0.32 \pm 0.05^{\mathrm{a}}$ & 4 & $0.31 \pm 0.18$ & 3 \\
\hline$I-49 \ldots$ & $-1.45 \pm 0.05^{\mathrm{a}}$ & 87 & $-1.52 \pm 0.05^{\mathrm{a}}$ & 12 & $0.58 \pm 0.05^{\mathrm{a}}$ & 2 & $0.00 \pm 0.05^{\mathrm{a}}$ & 3 & $0.38 \pm 0.12$ & 3 \\
\hline J37 & $-1.44 \pm 0.05^{\mathrm{a}}$ & 79 & $-1.52 \pm 0.05^{\mathrm{a}}$ & 11 & $0.15 \pm 0.10$ & 1 & $0.37 \pm 0.06$ & 4 & $0.09 \pm 0.10$ & 3 \\
\hline C41196_2632.................... & $-1.45 \pm 0.05^{\mathrm{a}}$ & 69 & $-1.51 \pm 0.05$ & 9 & $-0.01 \pm 0.10$ & 1 & $0.17 \pm 0.05^{\mathrm{a}}$ & 3 & $0.29 \pm 0.12$ & 3 \\
\hline 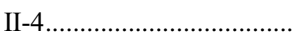 & $-1.54 \pm 0.05^{\mathrm{a}}$ & 69 & $-1.56 \pm 0.06$ & 10 & $0.26 \pm 0.10$ & 1 & $0.28 \pm 0.05^{\mathrm{a}}$ & 4 & $0.32 \pm 0.09$ & 3 \\
\hline IV-29 & $-1.42 \pm 0.05^{\mathrm{a}}$ & 69 & $-1.56 \pm 0.05^{\mathrm{a}}$ & 10 & $0.52 \pm 0.10$ & 1 & $-0.33 \pm 0.05^{\mathrm{a}}$ & 2 & $0.30 \pm 0.12$ & 3 \\
\hline 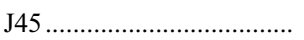 & $-1.44 \pm 0.05^{\mathrm{a}}$ & 69 & $-1.49 \pm 0.05$ & 10 & $0.16 \pm 0.10$ & 1 & $0.18 \pm 0.05^{\mathrm{a}}$ & 3 & $0.03 \pm 0.13$ & 3 \\
\hline $\mathrm{I}-5 \ldots \ldots \ldots \ldots \ldots \ldots$ & $-1.51 \pm 0.05^{\mathrm{a}}$ & 65 & $-1.59 \pm 0.06$ & 6 & & 0 & $0.49 \pm 0.05^{\mathrm{a}}$ & 3 & $0.27 \pm 0.13$ & 3 \\
\hline C41155_3103_..................... & $-1.47 \pm 0.05^{\mathrm{a}}$ & 70 & $-1.52 \pm 0.06$ & 3 & $-0.06 \pm 0.10$ & 1 & $0.35 \pm 0.05^{\mathrm{a}}$ & 3 & $-0.06 \pm 0.10$ & 1 \\
\hline C41148_3103..................... & $-1.49 \pm 0.05^{\mathrm{a}}$ & 59 & $-1.53 \pm 0.07$ & 2 & $0.55 \pm 0.05^{\mathrm{a}}$ & 2 & $-0.09 \pm 0.05^{\mathrm{a}}$ & 2 & $0.10 \pm 0.10$ & 1 \\
\hline 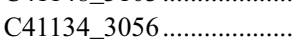 & $-1.44 \pm 0.05^{\mathrm{a}}$ & 60 & $-1.36 \pm 0.06$ & 7 & $0.24 \pm 0.05^{\mathrm{a}}$ & 2 & $-0.02 \pm 0.07$ & 2 & $0.26 \pm 0.17$ & 3 \\
\hline C40559_2839.................... & $-1.51 \pm 0.05^{\mathrm{a}}$ & 42 & $-1.58 \pm 0.10$ & 1 & $0.37 \pm 0.06$ & 2 & $0.15 \pm 0.08$ & 2 & $0.27 \pm 0.10$ & 1 \\
\hline C41101_3050....................... & $-1.46 \pm 0.05^{\mathrm{a}}$ & 56 & $-1.41 \pm 0.05$ & 6 & $0.37 \pm 0.10$ & 1 & $0.18 \pm 0.05^{\mathrm{a}}$ & 2 & $0.05 \pm 0.20$ & 3 \\
\hline C41099_3046 .................. & $-1.39 \pm 0.05^{\mathrm{a}}$ & 53 & $-1.33 \pm 0.06$ & 7 & $0.19 \pm 0.05^{\mathrm{a}}$ & 2 & $0.04 \pm 0.05^{\mathrm{a}}$ & 2 & $0.09 \pm 0.14$ & 3 \\
\hline C41135_3053 ..................... & $-1.51 \pm 0.05^{\mathrm{a}}$ & 44 & $-1.42 \pm 0.09$ & 4 & $0.35 \pm 0.05^{\mathrm{a}}$ & 2 & $-0.05 \pm 0.05^{\mathrm{a}}$ & 2 & $0.11 \pm 0.14$ & 3 \\
\hline C41133_2750.................... & $-1.53 \pm 0.05^{\mathrm{a}}$ & 33 & $\ldots$ & 0 & $0.26 \pm 0.05^{\mathrm{a}}$ & 2 & $-0.04 \pm 0.10$ & 1 & & 0 \\
\hline C40535_2819_............. & $-1.55 \pm 0.05^{\mathrm{a}}$ & 30 & & 0 & $0.38 \pm 0.07$ & 2 & $0.05 \pm 0.06$ & 2 & & 0 \\
\hline C40539_2813.................... & $-1.61 \pm 0.05^{\mathrm{a}}$ & 33 & & 0 & $0.25 \pm 0.07$ & 2 & $0.31 \pm 0.05^{\mathrm{a}}$ & 2 & & 0 \\
\hline C41135_2753 .................... & $-1.72 \pm 0.05$ & 15 & $\ldots$ & 0 & $0.14 \pm 0.13$ & 2 & $0.30 \pm 0.10$ & 1 & $\ldots$ & 0 \\
\hline
\end{tabular}

\footnotetext{
a The minimum value of 0.05 dex has been adopted; the nominal calculated value is smaller.
} 
TABLE 7B

Abundance Ratios for M13: Al to Ti

\begin{tabular}{|c|c|c|c|c|c|c|c|c|c|c|}
\hline Star & $\begin{array}{c}{[\mathrm{Al} / \mathrm{Fe}] \pm \sigma / \sqrt{N}} \\
(\mathrm{dex})\end{array}$ & $N$ & $\begin{array}{c}{[\mathrm{Si} / \mathrm{Fe}] \pm \sigma / \sqrt{N}} \\
(\mathrm{dex})\end{array}$ & $N$ & $\begin{array}{c}{[\mathrm{Ca} / \mathrm{Fe}] \pm \sigma / \sqrt{N}} \\
(\mathrm{dex})\end{array}$ & $N$ & $\begin{array}{c}{[\mathrm{Sc} / \mathrm{Fe}] \pm \sigma / \sqrt{N}} \\
(\mathrm{dex})\end{array}$ & $N$ & $\begin{array}{c}{[\mathrm{Ti} / \mathrm{Fe}] \pm \sigma / \sqrt{N}} \\
(\mathrm{dex})\end{array}$ & $N$ \\
\hline II-67.. & $0.64 \pm 0.10$ & 1 & $0.56 \pm 0.05$ & 9 & $-0.10 \pm 0.05^{\mathrm{a}}$ & 13 & $0.31 \pm 0.06$ & 7 & $-0.13 \pm 0.05^{\mathrm{a}}$ & 28 \\
\hline IV-25 & $0.85 \pm 0.10$ & 1 & $0.44 \pm 0.07$ & 8 & $0.04 \pm 0.05^{\mathrm{a}}$ & 13 & $0.27 \pm 0.05^{\mathrm{a}}$ & 7 & $0.04 \pm 0.05^{\mathrm{a}}$ & 27 \\
\hline 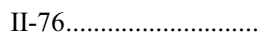 & $\leq 0.59$ & 1 & $0.41 \pm 0.05$ & 8 & $0.03 \pm 0.05^{\mathrm{a}}$ & 13 & $0.20 \pm 0.05^{\mathrm{a}}$ & 7 & $0.07 \pm 0.05^{\mathrm{a}}$ & 24 \\
\hline 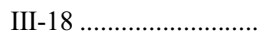 & $0.74 \pm 0.10$ & 1 & $0.36 \pm 0.05^{\mathrm{a}}$ & 8 & $0.10 \pm 0.05^{\mathrm{a}}$ & 13 & $0.21 \pm 0.05^{\mathrm{a}}$ & 7 & $0.09 \pm 0.05^{\mathrm{a}}$ & 22 \\
\hline 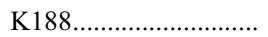 & $\leq 0.57$ & 1 & $0.27 \pm 0.05^{\mathrm{a}}$ & 9 & $0.11 \pm 0.05^{\mathrm{a}}$ & 13 & $0.16 \pm 0.05^{\mathrm{a}}$ & 7 & $0.13 \pm 0.05^{\mathrm{a}}$ & 15 \\
\hline 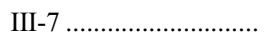 & $\leq 0.70$ & 1 & $0.37 \pm 0.05^{\mathrm{a}}$ & 8 & $0.16 \pm 0.05^{\mathrm{a}}$ & 13 & $0.19 \pm 0.05^{\mathrm{a}}$ & 7 & $0.04 \pm 0.05^{\mathrm{a}}$ & 11 \\
\hline I-18 & $\leq 0.76$ & 1 & $0.39 \pm 0.05^{\mathrm{a}}$ & 7 & $0.15 \pm 0.05^{\mathrm{a}}$ & 13 & $0.21 \pm 0.05^{\mathrm{a}}$ & 7 & $0.16 \pm 0.05^{\mathrm{a}}$ & 10 \\
\hline 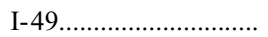 & $\leq 0.67$ & 1 & $0.32 \pm 0.05^{\mathrm{a}}$ & 7 & $0.15 \pm 0.05^{\mathrm{a}}$ & 13 & $0.13 \pm 0.06$ & 7 & $0.15 \pm 0.05$ & 10 \\
\hline 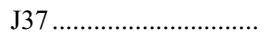 & $\leq 0.97$ & 1 & $0.31 \pm 0.05^{\mathrm{a}}$ & 6 & $0.12 \pm 0.05^{\mathrm{a}}$ & 12 & $0.09 \pm 0.05^{\mathrm{a}}$ & 7 & $0.11 \pm 0.07$ & 7 \\
\hline C41196_2632............ & $\ldots$ & 0 & $0.28 \pm 0.06$ & 6 & $0.12 \pm 0.05^{\mathrm{a}}$ & 12 & $0.14 \pm 0.05$ & 7 & $0.17 \pm 0.07$ & 5 \\
\hline 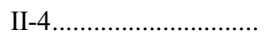 & $\ldots$ & 0 & $0.35 \pm 0.05$ & 6 & $0.13 \pm 0.05^{\mathrm{a}}$ & 12 & $0.12 \pm 0.05^{\mathrm{a}}$ & 7 & $0.31 \pm 0.08$ & 6 \\
\hline 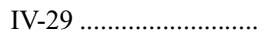 & $\ldots$ & 0 & $0.30 \pm 0.07$ & 6 & $0.03 \pm 0.05$ & 12 & $0.04 \pm 0.05$ & 7 & $0.20 \pm 0.05^{\mathrm{a}}$ & 6 \\
\hline 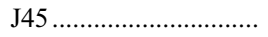 & $\ldots$ & 0 & $0.30 \pm 0.05^{\mathrm{a}}$ & 6 & $0.11 \pm 0.05^{\mathrm{a}}$ & 12 & $0.13 \pm 0.06$ & 7 & $0.24 \pm 0.05$ & 5 \\
\hline I-5 & $\ldots$ & 0 & $0.28 \pm 0.06$ & 5 & $0.12 \pm 0.05^{\mathrm{a}}$ & 12 & $0.12 \pm 0.05$ & 7 & $0.25 \pm 0.08$ & 5 \\
\hline C41155_3103 ….......... & $\ldots$ & 0 & $0.32 \pm 0.06$ & 5 & $0.22 \pm 0.05^{\mathrm{a}}$ & 16 & $0.11 \pm 0.05$ & 7 & $0.24 \pm 0.08$ & 6 \\
\hline C41148_3103 ….......... & $\ldots$ & 0 & $0.15 \pm 0.05^{\mathrm{a}}$ & 4 & $0.20 \pm 0.05^{\mathrm{a}}$ & 16 & $0.09 \pm 0.09$ & 5 & $0.30 \pm 0.07$ & 4 \\
\hline C41134_3056.............. & $\ldots$ & 0 & $0.22 \pm 0.07$ & 5 & $0.08 \pm 0.05^{\mathrm{a}}$ & 13 & $0.05 \pm 0.09$ & 2 & $-0.02 \pm 0.09$ & 4 \\
\hline C40559_2839............ & $\ldots$ & 0 & $0.09 \pm 0.05$ & 3 & $0.07 \pm 0.06$ & 14 & $0.10 \pm 0.08$ & 2 & $0.23 \pm 0.05$ & 2 \\
\hline C41101_3050............. & $\ldots$ & 0 & $0.35 \pm 0.06$ & 5 & $0.06 \pm 0.05^{\mathrm{a}}$ & 14 & $-0.02 \pm 0.05^{\mathrm{a}}$ & 2 & $0.22 \pm 0.10$ & 3 \\
\hline C41099_3046.............. & $\ldots$ & 0 & $0.10 \pm 0.05^{\mathrm{a}}$ & 2 & $0.14 \pm 0.05^{\mathrm{a}}$ & 13 & $0.11 \pm 0.05^{\mathrm{a}}$ & 2 & $0.29 \pm 0.05^{\mathrm{a}}$ & 6 \\
\hline C41135_3053 ….......... & $\ldots$ & 0 & $0.34 \pm 0.11$ & 2 & $0.20 \pm 0.05$ & 12 & $-0.08 \pm 0.11$ & 2 & $0.25 \pm 0.08$ & 3 \\
\hline C41133_2750............ & $\ldots$ & 0 & $\ldots$ & 0 & $0.20 \pm 0.06$ & 13 & $0.00 \pm 0.10$ & 1 & $\ldots$ & 0 \\
\hline C40535_2819............ & $\ldots$ & 0 & $\ldots$ & 0 & $0.24 \pm 0.05$ & 12 & $-0.03 \pm 0.10$ & 1 & $\ldots$ & 0 \\
\hline C40539_2813............. & $\ldots$ & 0 & $\ldots$ & 0 & $0.12 \pm 0.05^{\mathrm{a}}$ & 13 & $-0.02 \pm 0.10$ & 1 & $\ldots$ & 0 \\
\hline C41135_2753 „............ & $\ldots$ & 0 & $\ldots$ & 0 & $0.18 \pm 0.08$ & 9 & $0.36 \pm 0.10$ & 1 & $\ldots$ & 0 \\
\hline
\end{tabular}

a The minimum value of 0.05 dex has been adopted; the nominal calculated value is smaller.

TABLE 7C

Abundance Ratios for M13: V to Ni

\begin{tabular}{|c|c|c|c|c|c|c|c|c|c|c|}
\hline Star & $\begin{array}{c}{[\mathrm{V} / \mathrm{Fe}] \pm \sigma / \sqrt{N}} \\
(\mathrm{dex})\end{array}$ & $N$ & $\begin{array}{c}{[\mathrm{Cr} / \mathrm{Fe}] \pm \sigma / \sqrt{N}} \\
(\mathrm{dex})\end{array}$ & $N$ & $\begin{array}{c}{[\mathrm{Mn} / \mathrm{Fe}] \pm \sigma / \sqrt{N}} \\
(\mathrm{dex})\end{array}$ & $N$ & $\begin{array}{c}{[\mathrm{Co} / \mathrm{Fe}] \pm \sigma / \sqrt{N}} \\
(\mathrm{dex})\end{array}$ & $N$ & $\begin{array}{c}{[\mathrm{Ni} / \mathrm{Fe}] \pm \sigma / \sqrt{N}} \\
(\mathrm{dex})\end{array}$ & $N$ \\
\hline II-67 ............................. & $-0.33 \pm 0.05$ & 9 & $0.04 \pm 0.08$ & 10 & $-0.20 \pm 0.10$ & 4 & $-0.03 \pm 0.09$ & 4 & $-0.09 \pm 0.05^{\mathrm{a}}$ & 19 \\
\hline 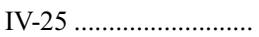 & $-0.14 \pm 0.05^{\mathrm{a}}$ & 9 & $0.10 \pm 0.07$ & 9 & $-0.26 \pm 0.13$ & 4 & $0.00 \pm 0.05$ & 4 & $-0.06 \pm 0.05^{\mathrm{a}}$ & 18 \\
\hline 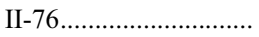 & $-0.19 \pm 0.05$ & 8 & $-0.11 \pm 0.07$ & 7 & $-0.36 \pm 0.05$ & 4 & $-0.03 \pm 0.05^{\mathrm{a}}$ & 4 & $-0.12 \pm 0.05^{\mathrm{a}}$ & 18 \\
\hline III-18 „.................... & $-0.11 \pm 0.05^{\mathrm{a}}$ & 8 & $-0.01 \pm 0.05$ & 7 & $-0.25 \pm 0.08$ & 4 & $-0.02 \pm 0.06$ & 4 & $-0.09 \pm 0.05^{\mathrm{a}}$ & 18 \\
\hline 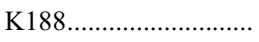 & $-0.16 \pm 0.05^{\mathrm{a}}$ & 7 & $-0.04 \pm 0.05$ & 7 & $-0.16 \pm 0.06$ & 4 & $-0.05 \pm 0.07$ & 3 & $-0.12 \pm 0.05^{\mathrm{a}}$ & 15 \\
\hline 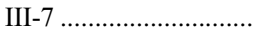 & $-0.09 \pm 0.10$ & 1 & $-0.06 \pm 0.06$ & 7 & $-0.14 \pm 0.05$ & 4 & $-0.02 \pm 0.08$ & 2 & $-0.19 \pm 0.05$ & 11 \\
\hline 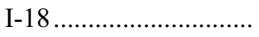 & $0.05 \pm 0.10$ & 1 & $-0.12 \pm 0.07$ & 5 & $-0.25 \pm 0.09$ & 4 & $-0.07 \pm 0.10$ & 1 & $-0.10 \pm 0.08$ & 11 \\
\hline I-49 & $-0.17 \pm 0.10$ & 1 & $-0.02 \pm 0.05$ & 5 & $-0.25 \pm 0.07$ & 3 & $0.08 \pm 0.10$ & 1 & $-0.08 \pm 0.05^{\mathrm{a}}$ & 11 \\
\hline 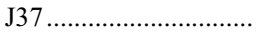 & $-0.07 \pm 0.10$ & 1 & $-0.06 \pm 0.05$ & 5 & $-0.44 \pm 0.09$ & 3 & $\ldots$ & 0 & $-0.13 \pm 0.06$ & 7 \\
\hline C41196_2632.. & $\ldots$ & 0 & $-0.03 \pm 0.05$ & 3 & $-0.25 \pm 0.05^{\mathrm{a}}$ & 3 & $\ldots$ & 0 & $-0.14 \pm 0.10$ & 5 \\
\hline II-4 .................. & $\ldots$ & 0 & $-0.11 \pm 0.09$ & 3 & $-0.55 \pm 0.05^{\mathrm{a}}$ & 3 & $\ldots$ & 0 & $-0.21 \pm 0.12$ & 5 \\
\hline 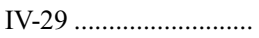 & $\ldots$ & 0 & $0.02 \pm 0.08$ & 3 & $-0.29 \pm 0.05^{\mathrm{a}}$ & 3 & $\ldots$ & 0 & $-0.02 \pm 0.09$ & 5 \\
\hline 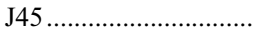 & $\ldots$ & 0 & $-0.14 \pm 0.08$ & 3 & $-0.31 \pm 0.08$ & 3 & $\ldots$ & 0 & $-0.06 \pm 0.09$ & 5 \\
\hline I-5 & $\ldots$ & 0 & $-0.07 \pm 0.06$ & 3 & $-0.40 \pm 0.06$ & 3 & $\ldots$ & 0 & $-0.10 \pm 0.10$ & 5 \\
\hline C41155_3103 „............ & $\ldots$ & 0 & $\ldots$ & 0 & $\ldots$ & 0 & $\ldots$ & 0 & $-0.08 \pm 0.06$ & 12 \\
\hline C41148_3103 „............ & $\cdots$ & 0 & $\ldots$ & 0 & $\ldots$ & 0 & $\cdots$ & 0 & $-0.16 \pm 0.05$ & 8 \\
\hline C41134_3056............. & $\ldots$ & 0 & $-0.11 \pm 0.05^{\mathrm{a}}$ & 3 & $-0.41 \pm 0.12$ & 3 & $\ldots$ & 0 & $0.08 \pm 0.14$ & 4 \\
\hline C40559_2839............. & $\ldots$ & 0 & $\ldots$ & 0 & $\ldots$ & 0 & $\ldots$ & 0 & $0.09 \pm 0.09$ & 4 \\
\hline C41101_3050 ............ & $\ldots$ & 0 & $-0.19 \pm 0.06$ & 3 & $-0.50 \pm 0.05^{\mathrm{a}}$ & 3 & $\ldots$ & 0 & $-0.18 \pm 0.12$ & 4 \\
\hline C41099_3046............. & $\ldots$ & 0 & $-0.15 \pm 0.11$ & 3 & $-0.38 \pm 0.10$ & 3 & $\ldots$ & 0 & $-0.05 \pm 0.10$ & 3 \\
\hline C41135_3053 ............. & $\ldots$ & 0 & $0.06 \pm 0.05$ & 3 & $-0.38 \pm 0.13$ & 3 & $\ldots$ & 0 & $-0.01 \pm 0.05^{\mathrm{a}}$ & 2 \\
\hline C41133_2750.............. & $\cdots$ & 0 & $\ldots$ & 0 & $\ldots$ & 0 & $\cdots$ & 0 & $-0.34 \pm 0.10$ & 1 \\
\hline C40535_2819............ & $\ldots$ & 0 & $\ldots$ & 0 & $\ldots$ & 0 & $\ldots$ & 0 & $-0.14 \pm 0.10$ & 1 \\
\hline C40539_2813 ............. & $\ldots$ & 0 & $\ldots$ & 0 & $\ldots$ & 0 & $\ldots$ & 0 & $-0.10 \pm 0.09$ & 2 \\
\hline C41135_2753 „............ & $\ldots$ & 0 & $\ldots$ & 0 & $\ldots$ & 0 & $\ldots$ & 0 & $\ldots$ & 0 \\
\hline
\end{tabular}

a The minimum value of 0.05 dex has been adopted; the nominal calculated value is smaller. 
TABLE 7D

Abundance Ratios for M13: $\mathrm{Cu}$ to Ba

\begin{tabular}{|c|c|c|c|c|c|c|c|c|c|c|}
\hline Star & $\begin{array}{c}{[\mathrm{Cu} / \mathrm{Fe}] \pm \sigma / \sqrt{N}} \\
(\mathrm{dex})\end{array}$ & $N$ & $\begin{array}{c}{[\mathrm{Zn} / \mathrm{Fe}] \pm \sigma / \sqrt{N}} \\
(\mathrm{dex})\end{array}$ & $N$ & $\begin{array}{c}{[\mathrm{Y} / \mathrm{Fe}] \pm \sigma / \sqrt{N}} \\
(\mathrm{dex})\end{array}$ & $N$ & $\begin{array}{c}{[\mathrm{Zr} / \mathrm{Fe}] \pm \sigma / \sqrt{N}} \\
(\operatorname{dex})\end{array}$ & $N$ & $\begin{array}{c}{[\mathrm{Ba} / \mathrm{Fe}] \pm \sigma / \sqrt{N}} \\
(\mathrm{dex})\end{array}$ & $N$ \\
\hline II-67.............................. & $-0.55 \pm 0.13$ & 2 & $0.17 \pm 0.14$ & 2 & $-0.08 \pm 0.11$ & 5 & $-0.37 \pm 0.05$ & 3 & $0.43 \pm 0.05^{\mathrm{a}}$ & 3 \\
\hline 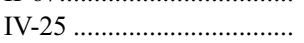 & $-0.62 \pm 0.06$ & 2 & $0.02 \pm 0.05^{\mathrm{a}}$ & 2 & $-0.18 \pm 0.07$ & 5 & $-0.12 \pm 0.05^{\mathrm{a}}$ & 3 & $0.28 \pm 0.05$ & 3 \\
\hline 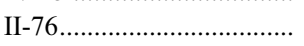 & $-0.62 \pm 0.12$ & 2 & $0.04 \pm 0.05^{\mathrm{a}}$ & 2 & $-0.26 \pm 0.05^{\mathrm{a}}$ & 4 & $0.06 \pm 0.06$ & 3 & $0.38 \pm 0.06$ & 3 \\
\hline 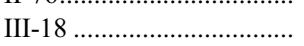 & $-0.61 \pm 0.13$ & 2 & $-0.05 \pm 0.05^{\mathrm{a}}$ & 2 & $-0.26 \pm 0.06$ & 5 & $0.05 \pm 0.07$ & 2 & $0.36 \pm 0.05$ & 3 \\
\hline K188. & $-0.70 \pm 0.05^{\mathrm{a}}$ & 2 & $0.08 \pm 0.05^{\mathrm{a}}$ & 2 & $-0.13 \pm 0.05$ & 4 & $\ldots$ & 0 & $0.44 \pm 0.05^{\mathrm{a}}$ & 3 \\
\hline 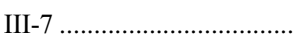 & $-0.73 \pm 0.05^{\mathrm{a}}$ & 2 & $0.16 \pm 0.05^{\mathrm{a}}$ & 2 & $-0.21 \pm 0.05^{\mathrm{a}}$ & 5 & $\ldots$ & 0 & $0.44 \pm 0.05$ & 3 \\
\hline I-18 ........................................... & $-0.61 \pm 0.08$ & 2 & $0.09 \pm 0.05^{\mathrm{a}}$ & 2 & $-0.25 \pm 0.05$ & 4 & $\ldots$ & 0 & $0.37 \pm 0.06$ & 3 \\
\hline 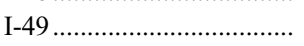 & $-0.68 \pm 0.11$ & 2 & $0.06 \pm 0.05^{\mathrm{a}}$ & 2 & $-0.33 \pm 0.09$ & 4 & $\ldots$ & 0 & $0.28 \pm 0.05$ & 3 \\
\hline 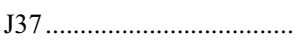 & $-0.80 \pm 0.10$ & 1 & $-0.02 \pm 0.06$ & 2 & $-0.16 \pm 0.08$ & 4 & $\ldots$ & 0 & $0.30 \pm 0.05$ & 3 \\
\hline C41196_2632 .................... & $-0.68 \pm 0.10$ & 1 & $-0.14 \pm 0.05$ & 2 & $-0.26 \pm 0.08$ & 4 & $\ldots$ & 0 & $0.33 \pm 0.10$ & 3 \\
\hline 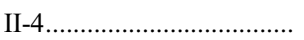 & $-0.79 \pm 0.10$ & 1 & $-0.28 \pm 0.05^{\mathrm{a}}$ & 2 & $-0.17 \pm 0.05$ & 4 & $\ldots$ & 0 & $0.37 \pm 0.09$ & 3 \\
\hline 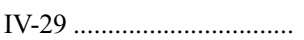 & $-0.63 \pm 0.10$ & 1 & $-0.04 \pm 0.07$ & 2 & $-0.26 \pm 0.05$ & 4 & $\ldots$ & 0 & $0.20 \pm 0.05^{\mathrm{a}}$ & 3 \\
\hline 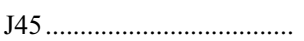 & $-0.52 \pm 0.10$ & 1 & $0.00 \pm 0.05^{\mathrm{a}}$ & 2 & $-0.29 \pm 0.05^{\mathrm{a}}$ & 4 & $\ldots$ & 0 & $0.15 \pm 0.05^{\mathrm{a}}$ & 3 \\
\hline I-5 ………………........... & $-0.56 \pm 0.10$ & 1 & $0.12 \pm 0.05^{\mathrm{a}}$ & 2 & $0.53 \pm 0.05^{\mathrm{a}}$ & 3 & $\ldots$ & 0 & $0.58 \pm 0.06$ & 3 \\
\hline 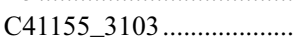 & $\ldots$ & 0 & $\ldots$ & 0 & $\ldots$ & 0 & & 0 & $0.12 \pm 0.08$ & 3 \\
\hline C41148_3103_.................... & $\ldots$ & 0 & $\ldots$ & 0 & $\ldots$ & 0 & $\ldots$ & 0 & $0.01 \pm 0.08$ & 3 \\
\hline C41134_3056_.................... & $\ldots$ & 0 & $-0.11 \pm 0.10$ & 1 & $-0.14 \pm 0.07$ & 4 & $\ldots$ & 0 & $0.37 \pm 0.05$ & 3 \\
\hline C40559_2839..................... & $\ldots$ & 0 & $\ldots$ & 0 & $\ldots$ & 0 & $\ldots$ & 0 & $0.06 \pm 0.08$ & 3 \\
\hline C41101_3050.................... & $\ldots$ & 0 & $-0.11 \pm 0.10$ & 1 & $-0.29 \pm 0.07$ & 4 & $\ldots$ & 0 & $0.22 \pm 0.06$ & 3 \\
\hline 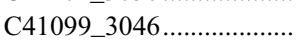 & $\ldots$ & 0 & $-0.09 \pm 0.10$ & 1 & $-0.13 \pm 0.05^{\mathrm{a}}$ & 3 & $\ldots$ & 0 & $0.39 \pm 0.05$ & 3 \\
\hline C41135_3053 ….................. & $\ldots$ & 0 & $0.00 \pm 0.10$ & 1 & $-0.19 \pm 0.10$ & 1 & $\cdots$ & 0 & $0.27 \pm 0.05^{\mathrm{a}}$ & 3 \\
\hline 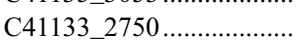 & $\cdots$ & 0 & $\ldots$ & 0 & $\ldots$ & 0 & $\ldots$ & 0 & $0.16 \pm 0.13$ & 2 \\
\hline C40535_2819.................... & $\ldots$ & 0 & $\ldots$ & 0 & $\ldots$ & 0 & $\ldots$ & 0 & $-0.04 \pm 0.11$ & 2 \\
\hline C40539_2813...................... & $\ldots$ & 0 & $\ldots$ & 0 & $\ldots$ & 0 & $\ldots$ & 0 & $0.03 \pm 0.06$ & 2 \\
\hline C41135_2753 ..................... & $\ldots$ & 0 & $\ldots$ & 0 & $\ldots$ & 0 & $\cdots$ & 0 & $0.09 \pm 0.08$ & 2 \\
\hline
\end{tabular}

a The minimum value of 0.05 dex has been adopted; the nominal calculated value is smaller.

TABLE 7E

Abundance Ratios for M13: La to Dy

\begin{tabular}{|c|c|c|c|c|c|c|c|c|}
\hline Star & $\begin{array}{c}{[\mathrm{La} / \mathrm{Fe}] \pm \sigma / \sqrt{N}} \\
(\operatorname{dex})\end{array}$ & $N$ & $\begin{array}{c}{[\mathrm{Nd} / \mathrm{Fe}] \pm \sigma / \sqrt{N}} \\
(\operatorname{dex})\end{array}$ & $N$ & $\begin{array}{c}{[\mathrm{Eu} / \mathrm{Fe}] \pm \sigma / \sqrt{N}} \\
(\operatorname{dex})\end{array}$ & $N$ & {$[\mathrm{Dy} / \mathrm{Fe}]$} & $N$ \\
\hline II-67 ........................... & $0.12 \pm 0.06$ & 3 & $0.41 \pm 0.05$ & 7 & $0.56 \pm 0.10$ & 1 & $-0.03 \pm 0.10$ & 1 \\
\hline IV-25 ............................. & $0.20 \pm 0.10$ & 3 & $0.40 \pm 0.05^{\mathrm{a}}$ & 7 & $0.59 \pm 0.10$ & 1 & $0.09 \pm 0.10$ & 1 \\
\hline II-76 & $0.11 \pm 0.10$ & 3 & $0.21 \pm 0.05^{\mathrm{a}}$ & 7 & $0.30 \pm 0.10$ & 1 & $0.10 \pm 0.10$ & 1 \\
\hline III-18 & $0.09 \pm 0.09$ & 3 & $0.31 \pm 0.06$ & 7 & $0.58 \pm 0.10$ & 1 & $0.22 \pm 0.10$ & 1 \\
\hline K188............................ & $0.09 \pm 0.09$ & 3 & $0.27 \pm 0.05^{\mathrm{a}}$ & 7 & $0.67 \pm 0.10$ & 1 & $\ldots$ & 0 \\
\hline III-7 ............................ & $0.21 \pm 0.05$ & 3 & $0.24 \pm 0.07$ & 6 & $0.71 \pm 0.10$ & 1 & $\ldots$ & 0 \\
\hline I-18 & $0.11 \pm 0.10$ & 2 & $0.12 \pm 0.07$ & 6 & $0.67 \pm 0.10$ & 1 & $\ldots$ & 0 \\
\hline 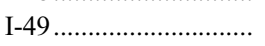 & $0.06 \pm 0.14$ & 2 & $0.23 \pm 0.05^{\mathrm{a}}$ & 7 & $0.52 \pm 0.10$ & 1 & $\ldots$ & 0 \\
\hline 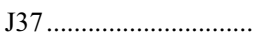 & $-0.05 \pm 0.10$ & 1 & $0.33 \pm 0.06$ & 5 & $0.68 \pm 0.10$ & 1 & $\ldots$ & 0 \\
\hline C41196_2632............. & $0.04 \pm 0.10$ & 1 & $0.16 \pm 0.07$ & 5 & $0.48 \pm 0.10$ & 1 & $\ldots$ & 0 \\
\hline II-4 ................................ & $0.28 \pm 0.10$ & 1 & $0.16 \pm 0.09$ & 3 & $\ldots$ & 0 & $\ldots$ & 0 \\
\hline 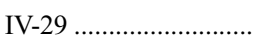 & $0.28 \pm 0.05^{\mathrm{a}}$ & 2 & $0.35 \pm 0.05^{\mathrm{a}}$ & 6 & $0.59 \pm 0.10$ & 1 & $\ldots$ & 0 \\
\hline $\mathrm{J} 45$ & $\ldots$ & 0 & $0.28 \pm 0.05$ & 5 & $0.51 \pm 0.10$ & 1 & $\ldots$ & 0 \\
\hline I-5 .............................. & $0.23 \pm 0.10$ & 1 & $0.47 \pm 0.05$ & 5 & $\ldots$ & 0 & $\ldots$ & 0 \\
\hline C41155_3103............... & $\ldots$ & 0 & $\ldots$ & 0 & $\ldots$ & 0 & $\ldots$ & 0 \\
\hline C41148_3103............. & $\ldots$ & 0 & $\ldots$ & 0 & $\ldots$ & 0 & $\ldots$ & 0 \\
\hline C41134_3056............. & $\ldots$ & 0 & $\ldots$ & 0 & $\ldots$ & 0 & $\ldots$ & 0 \\
\hline C40559_2839 ............ & $\ldots$ & 0 & $\ldots$ & 0 & $\ldots$ & 0 & $\ldots$ & 0 \\
\hline C41101_3050............. & $\ldots$ & 0 & $\ldots$ & 0 & $\ldots$ & 0 & $\ldots$ & 0 \\
\hline C41099_3046 ............. & $\ldots$ & 0 & $\ldots$ & 0 & $\ldots$ & 0 & $\ldots$ & 0 \\
\hline C41135_3053.............. & $\ldots$ & 0 & $\ldots$ & 0 & $\ldots$ & 0 & $\ldots$ & 0 \\
\hline C41133_2750.............. & $\ldots$ & 0 & $\ldots$ & 0 & $\ldots$ & 0 & $\ldots$ & 0 \\
\hline C40535_2819 ............. & $\ldots$ & 0 & $\ldots$ & 0 & $\ldots$ & 0 & $\ldots$ & 0 \\
\hline C40539_2813 .............. & $\ldots$ & 0 & $\ldots$ & 0 & $\ldots$ & 0 & $\ldots$ & 0 \\
\hline C41135_2753.............. & $\ldots$ & 0 & $\ldots$ & 0 & $\ldots$ & 0 & $\ldots$ & 0 \\
\hline
\end{tabular}

\footnotetext{
${ }^{\text {a }}$ The minimum value of 0.05 dex has been adopted; the nominal calculated value is smaller.
} 
TABLE 8

Sensitivity of Abundances

\begin{tabular}{|c|c|c|c|c|c|}
\hline Species & $\begin{array}{c}\Delta(\mathrm{EW}) \\
10 \%{ }^{\mathrm{a}} \\
(\mathrm{dex})\end{array}$ & $\begin{array}{c}\Delta\left(T_{\mathrm{eff}}\right) \\
+100 \mathrm{~K}^{\mathrm{b}} \\
(\mathrm{dex})\end{array}$ & $\begin{array}{l}\Delta(\log g) \\
+0.2 \operatorname{dex} \\
\quad(\operatorname{dex})\end{array}$ & $\begin{array}{c}\Delta(v t) \\
+0.4 /-0.2 \mathrm{~km} \mathrm{~s}^{-1} \\
(\mathrm{dex})\end{array}$ & $\begin{array}{l}\Delta([\mathrm{Fe} / \mathrm{H}]) \\
+0.2 \operatorname{dex} \\
\quad(\operatorname{dex})\end{array}$ \\
\hline \multicolumn{6}{|l|}{ O I: } \\
\hline 4250/1.0/1.6 .......................... & 0.05 & -0.01 & 0.09 & $-0.01 / 0.00$ & 0.08 \\
\hline $5250 / 3.0 / 1.3 \ldots \ldots \ldots \ldots \ldots \ldots \ldots \ldots$ & 0.06 & -0.09 & 0.07 & $-0.01 /+0.01$ & -0.01 \\
\hline $5750 / 3.5 / 2.0 \ldots \ldots \ldots \ldots \ldots \ldots \ldots$ & 0.06 & -0.08 & 0.07 & $-0.02 /+0.01$ & -0.01 \\
\hline \multicolumn{6}{|l|}{ Na I: } \\
\hline 4250/1.0/1.6 ..................... & 0.07 & 0.09 & -0.02 & $-0.02 /+0.02$ & -0.01 \\
\hline $5250 / 3.0 / 1.3 \ldots \ldots \ldots \ldots \ldots \ldots \ldots \ldots$ & 0.06 & 0.06 & -0.03 & $-0.01 /+0.01$ & 0.00 \\
\hline $5750 / 3.5 / 2.0 \ldots \ldots \ldots \ldots \ldots \ldots \ldots \ldots \ldots \ldots$ & 0.05 & 0.04 & 0.00 & $-0.01 / 0.00$ & 0.00 \\
\hline \multicolumn{6}{|l|}{ Mg I: } \\
\hline 4250/1.0/1.6 .......................... & 0.17 & 0.10 & -0.05 & $-0.16 /+0.07$ & 0.00 \\
\hline $5250 / 3.0 / 1.3 \ldots \ldots \ldots \ldots \ldots \ldots \ldots \ldots$ & 0.12 & 0.08 & -0.05 & $-0.06 /+0.03$ & 0.01 \\
\hline \multicolumn{6}{|l|}{ Si I: } \\
\hline 4250/1.0/1.6 ..................... & 0.06 & 0.01 & 0.03 & $-0.02 /+0.01$ & -0.03 \\
\hline $5250 / 3.0 / 1.3 \ldots \ldots \ldots \ldots \ldots \ldots \ldots \ldots$ & 0.06 & 0.03 & 0.01 & $-0.01 /+0.01$ & 0.01 \\
\hline \multicolumn{6}{|l|}{ Ca I: } \\
\hline 4250/1.0/1.6 .......................... & 0.16 & 0.13 & -0.02 & $-0.16 /+0.10$ & -0.02 \\
\hline $5250 / 3.0 / 1.3 \ldots \ldots \ldots \ldots \ldots \ldots \ldots$ & 0.10 & 0.07 & -0.02 & $-0.08 /+0.04$ & 0.00 \\
\hline $5750 / 3.5 / 2.0 \ldots \ldots \ldots \ldots \ldots \ldots \ldots \ldots$ & 0.09 & 0.06 & -0.01 & $-0.06 /+0.03$ & 0.00 \\
\hline \multicolumn{6}{|l|}{ Sc II: } \\
\hline 4250/1.0/1.6 ...................... & 0.13 & -0.02 & 0.08 & $-0.12 /+0.07$ & 0.06 \\
\hline $5250 / 3.0 / 1.3 \ldots \ldots \ldots \ldots \ldots \ldots \ldots \ldots \ldots \ldots \ldots \ldots \ldots . .$. & 0.09 & 0.03 & 0.08 & $-0.05 /+0.04$ & 0.04 \\
\hline $5750 / 3.5 / 2.0 \ldots \ldots \ldots \ldots \ldots \ldots \ldots \ldots$ & 0.06 & 0.03 & 0.07 & $-0.02 /+0.02$ & 0.02 \\
\hline \multicolumn{6}{|l|}{ Ti I: } \\
\hline 4250/1.0/1.6 ...................... & 0.11 & 0.19 & -0.01 & $-0.09 /+0.06$ & -0.02 \\
\hline $5250 / 3.0 / 1.3 \ldots \ldots \ldots \ldots \ldots \ldots \ldots \ldots$ & 0.08 & 0.11 & -0.01 & $-0.05 /+0.03$ & 0.00 \\
\hline \multicolumn{6}{|l|}{ Ti II: } \\
\hline 4250/1.0/1.6 ......................... & 0.20 & -0.02 & 0.06 & $-0.21 /+0.14$ & 0.12 \\
\hline 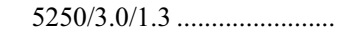 & 0.10 & 0.03 & 0.08 & $-0.05 /+0.04$ & 0.04 \\
\hline \multicolumn{6}{|l|}{ V I: } \\
\hline 4250/1.0/1.6 ....................... & 0.06 & 0.22 & 0.00 & $-0.03 /+0.02$ & -0.02 \\
\hline \multicolumn{6}{|l|}{ Cr I: } \\
\hline 4250/1.0/1.6 ......................... & 0.13 & 0.14 & -0.02 & $-0.15 /+0.08$ & -0.02 \\
\hline 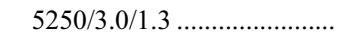 & 0.14 & 0.12 & -0.02 & $-0.12 /+0.08$ & 0.00 \\
\hline \multicolumn{6}{|l|}{ Mn I: } \\
\hline 4250/1.0/1.6 ..................... & 0.19 & 0.16 & -0.02 & $-0.23 /+0.13$ & -0.01 \\
\hline 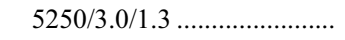 & 0.12 & 0.10 & -0.01 & $-0.10 /+0.06$ & 0.00 \\
\hline \multicolumn{6}{|l|}{ Fe I: } \\
\hline 4250/1.0/1.6 .......................... & 0.12 & 0.11 & 0.00 & $-0.13 /+0.07$ & 0.01 \\
\hline $5250 / 3.0 / 1.3 \ldots \ldots \ldots \ldots \ldots \ldots \ldots \ldots \ldots \ldots \ldots \ldots \ldots$ & 0.11 & 0.10 & -0.02 & $-0.09 /+0.05$ & 0.00 \\
\hline $5750 / 3.5 / 2.0 \ldots \ldots \ldots \ldots \ldots \ldots \ldots$ & 0.09 & 0.08 & 0.00 & $-0.05 /+0.03$ & 0.01 \\
\hline \multicolumn{6}{|l|}{ Fe II: } \\
\hline 4250/1.0/1.6 ....................... & 0.11 & -0.08 & 0.08 & $-0.12 /+0.06$ & 0.07 \\
\hline 5250/3.0/1.3 ...................... & 0.08 & -0.01 & 0.08 & $-0.05 /+0.03$ & 0.03 \\
\hline \multicolumn{6}{|l|}{ Co I: } \\
\hline 4250/1.0/1.6 ...................... & 0.05 & 0.13 & 0.02 & $-0.02 /+0.01$ & 0.02 \\
\hline Ni I: & & & & & \\
\hline 4250/1.0/1.6 .......................... & 0.08 & 0.09 & 0.03 & $-0.07 /+0.04$ & 0.02 \\
\hline $5250 / 3.0 / 1.3 \ldots \ldots \ldots \ldots \ldots \ldots \ldots \ldots$ & 0.07 & 0.10 & 0.00 & $-0.05 /+0.02$ & 0.00 \\
\hline $5750 / 3.5 / 2.0 \ldots \ldots \ldots \ldots \ldots \ldots \ldots \ldots$ & 0.06 & 0.06 & 0.00 & $-0.02 /+0.02$ & 0.01 \\
\hline $\mathrm{Cu}$ I: & & & & & \\
\hline 4250/1.0/1.6 ....................... & 0.16 & 0.14 & 0.03 & $-0.17 /+0.12$ & 0.02 \\
\hline Zn I: & & & & & \\
\hline 4250/1.0/1.6 ......................... & 0.14 & -0.05 & 0.04 & $-0.13 /+0.10$ & 0.04 \\
\hline 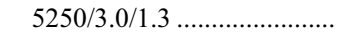 & 0.07 & 0.04 & 0.04 & $-0.05 /+0.03$ & 0.02 \\
\hline Y II: & & & & & \\
\hline 4250/1.0/1.6 .......................... & 0.17 & -0.01 & 0.07 & $-0.18 /+0.13$ & -0.06 \\
\hline $5250 / 3.0 / 1.3 \ldots \ldots \ldots \ldots \ldots \ldots \ldots \ldots$ & 0.07 & 0.04 & 0.08 & $-0.03 /+0.03$ & 0.04 \\
\hline Zr I: & & & & & \\
\hline 4250/1.0/1.6 ...................... & 0.05 & 0.25 & 0.00 & $0.00 /+0.01$ & -0.02 \\
\hline Ва II: & & & & & \\
\hline 4250/1.0/1.6 ...................... & 0.23 & 0.04 & 0.06 & $-0.36 /+0.20$ & 0.06 \\
\hline 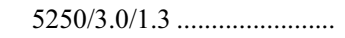 & 0.17 & 0.07 & 0.05 & $-0.23 /+0.15$ & 0.04 \\
\hline $5750 / 3.5 / 2.0 \ldots \ldots \ldots \ldots \ldots \ldots \ldots$ & 0.12 & 0.06 & 0.06 & $-0.10 /+0.07$ & 0.02 \\
\hline
\end{tabular}


TABLE 8-Continued

\begin{tabular}{|c|c|c|c|c|c|}
\hline Species & $\begin{array}{c}\Delta(\mathrm{EW}) \\
10 \%{ }^{\mathrm{a}} \\
(\mathrm{dex})\end{array}$ & $\begin{array}{c}\Delta\left(T_{\text {eff }}\right) \\
+100 \mathrm{~K}^{\mathrm{b}} \\
(\mathrm{dex})\end{array}$ & $\begin{array}{c}\Delta(\log g) \\
+0.2 \mathrm{dex} \\
\quad(\operatorname{dex})\end{array}$ & $\begin{array}{c}\Delta(v t) \\
+0.4 /-0.2 \mathrm{~km} \mathrm{~s}^{-1} \\
(\mathrm{dex})\end{array}$ & $\begin{array}{c}\Delta([\mathrm{Fe} / \mathrm{H}]) \\
+0.2 \mathrm{dex} \\
\quad(\operatorname{dex})\end{array}$ \\
\hline \multicolumn{6}{|l|}{ La II: } \\
\hline 4250/1.0/1.6 …........................ & 0.06 & 0.02 & 0.08 & $-0.02 /+0.02$ & 0.07 \\
\hline \multicolumn{6}{|l|}{$\mathrm{Nd}$ II: } \\
\hline 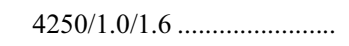 & 0.09 & 0.03 & 0.07 & $-0.07 /+0.06$ & 0.06 \\
\hline \multicolumn{6}{|l|}{ Eu II: } \\
\hline 4250/1.0/1.6 ........................ & 0.06 & -0.01 & 0.08 & $-0.03 /+0.02$ & 0.07 \\
\hline \multicolumn{6}{|l|}{ Dy II: } \\
\hline 4250/1.0/1.6 ….................... & 0.05 & 0.03 & 0.08 & $-0.01 /+0.01$ & 0.07 \\
\hline
\end{tabular}

${ }^{\text {a }}$ This is an underestimate of the uncertainty for the weakest lines.

${ }^{\mathrm{b}}$ The $T_{\text {eff }}$ uncertainty is a function of $T_{\text {eff }}$; see $\S 5$.

work with those from previous investigations (most of which relied on $B-V$ colors) for the stars in common in M3 and in M13 with the analyses of Cohen (1978), Kraft et al. (1992, 1997), Cavallo \& Nagar (2000), and Sneden et al. (2004) is shown in Table 11 . The agreement is very gratifying. No difference exceeds $100 \mathrm{~K}$, and most are $50 \mathrm{~K}$ or less.

A comparison of the mean abundance ratios of large sample of bright giants in M3 (M13) analyzed by Sneden et al. (2004) with our determinations is given in Table 12. The results are encouraging. The differences are given in the last column of the table. The deduced $[\mathrm{Fe} / \mathrm{H}](\mathrm{Fe}$ I) between the current work and that of Sneden et al. (2004) differs by 0.23 (0.13) dex, while the difference using $\mathrm{Fe}$ II is only $0.12(0.03)$ dex. The largest difference in abundance ratios $[\mathrm{X} / \mathrm{Fe}]$ for $\mathrm{M} 3$ is $0.24 \mathrm{dex}$ (for $\mathrm{Sc}$ II), with only three species ( $\mathrm{O}$ I, $\mathrm{Mg}$ I, and $\mathrm{Sc}$ II) having differences exceeding 0.15 dex. For $\mathrm{M} 13,[\mathrm{O} / \mathrm{Fe}]$ and $[\mathrm{Mg} / \mathrm{Fe}]$ both have differences exceeding 0.15 dex. Part of the difference in $\mathrm{O}$ arises because Sneden et al. (2004) adopt a solar O abundance of $8.93 \mathrm{dex}, 0.08 \mathrm{dex}$ higher than we do. In principle, it could also be produced, at least partially, if the amplitude of the star-to-star variations of $\mathrm{O}$ were a function of stellar luminosity, or equivalently $T_{\text {eff }}$, given the differences in mean luminosity of the two samples. Other differences in the details of the analysis may enter as well. For example, the difference in $\mathrm{Ca}$ appears to

TABLE 9

Mean Abundances and Abundance Spreads for Stars in M3

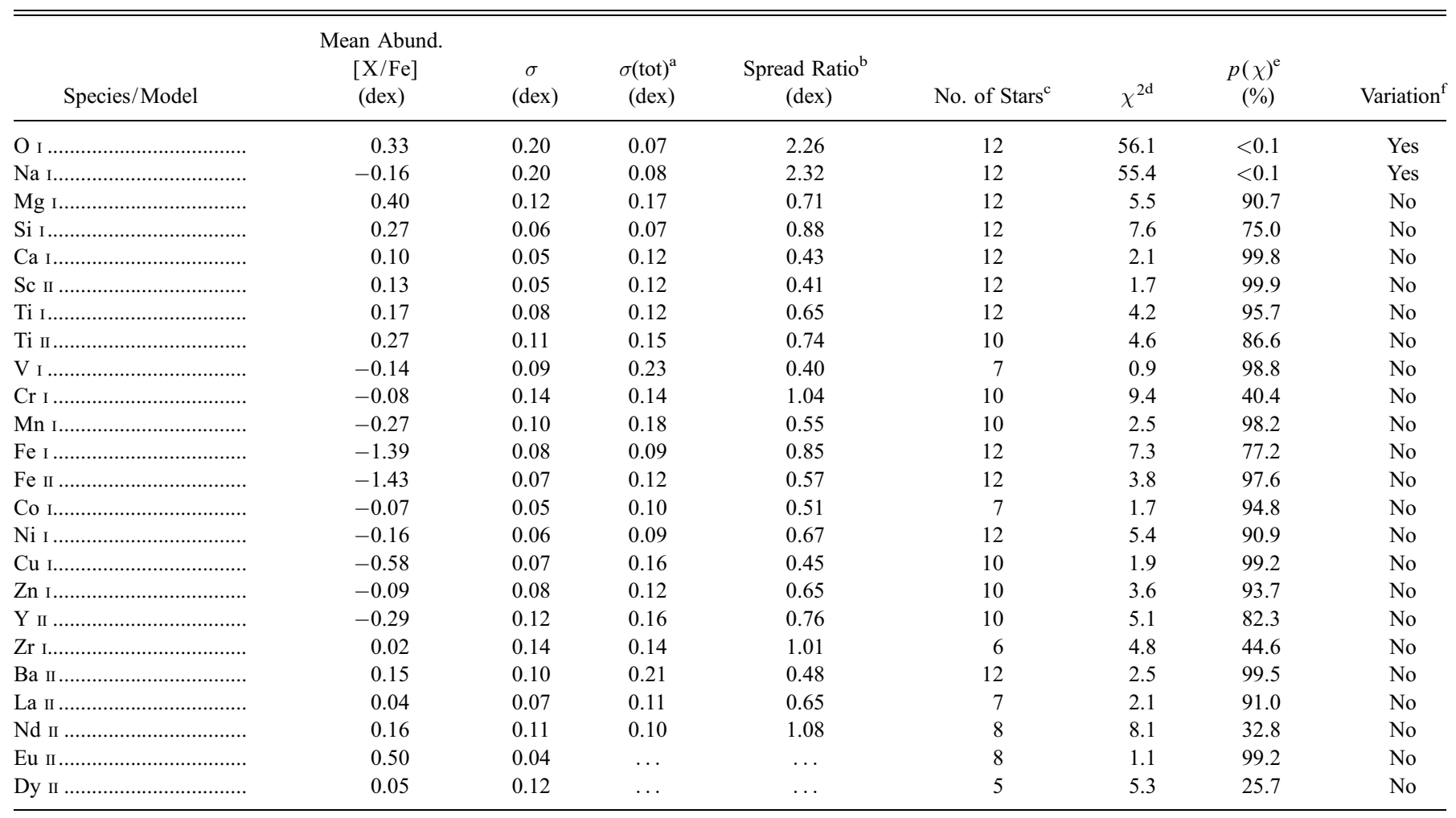

${ }^{\text {a }}$ The value $\sigma($ tot $)$ is defined in $\S 6.2$.

b This is the ratio of $\sigma$ for the M3 sample stars to $\sigma$ (tot). See text.

c The number of stars in the M3 sample in which lines of this species were detected.

${ }^{\mathrm{d}}$ Defined as $\chi^{2}=\sum\left(y_{i}-\bar{y}\right)^{2} / \sigma_{i}^{2}$, where $\bar{y}=\sum\left(y_{i} / \sigma_{i}^{2}\right) / \sum\left(1 / \sigma_{i}^{2}\right)$. A minimum $\sigma_{i}=\sigma$ (tot $)$ was adopted.

e The value $p(\chi)$ is the probability of exceeding $\chi^{2}$ by chance.

${ }^{\mathrm{f}}$ If $p(\chi)<0.1 \%$, there is abundance variation; if $p(\chi)>0.5 \%$, there is no variation. 
TABLE 10

Mean Abundances and Abundance Spreads for Stars in M13

\begin{tabular}{|c|c|c|c|c|c|c|c|c|}
\hline Species & $\begin{array}{c}\text { Mean Abund. } \\
{[\mathrm{X} / \mathrm{Fe}]} \\
(\mathrm{dex})\end{array}$ & $\begin{array}{c}\sigma \\
(\mathrm{dex})\end{array}$ & $\begin{array}{r}\sigma(\text { tot })^{\mathrm{a}} \\
\quad(\mathrm{dex})\end{array}$ & $\begin{array}{c}\text { Spread Ratio } \\
\text { (dex) }\end{array}$ & No. of Stars ${ }^{\mathrm{c}}$ & $\chi^{2 \mathrm{~d}}$ & $\begin{array}{l}p(\chi)^{\mathrm{e}} \\
\quad(\%)\end{array}$ & Variation $^{\mathrm{f}}$ \\
\hline O I & 0.18 & 0.37 & 0.10 & 3.70 & 24 & 319 & $<0.1$ & Yes \\
\hline 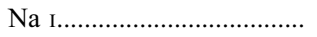 & 0.14 & 0.22 & 0.08 & 2.75 & 25 & 174 & $<0.1$ & Yes \\
\hline 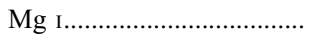 & 0.24 & 0.15 & 0.18 & 0.83 & 21 & 14.2 & 81.9 & No \\
\hline 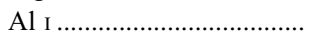 & $0.74^{\mathrm{g}}$ & 0.11 & $\ldots$ & $\ldots$ & 3 & $\ldots$ & $\ldots$ & $\ldots$ \\
\hline 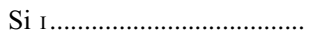 & 0.31 & 0.11 & 0.08 & 1.37 & 21 & 36.9 & 1.9 & No \\
\hline 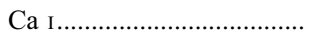 & 0.12 & 0.07 & 0.12 & 0.58 & 25 & 9.1 & 99.7 & No \\
\hline 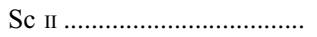 & 0.12 & 0.11 & 0.12 & 0.92 & 25 & 19.2 & 74.4 & No \\
\hline 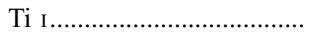 & 0.16 & 0.11 & 0.13 & 0.85 & 21 & 15.4 & 75.4 & No \\
\hline 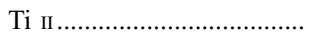 & 0.31 & 0.12 & 0.15 & 0.80 & 18 & 9.6 & 92.0 & No \\
\hline 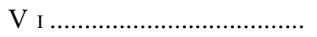 & -0.13 & 0.10 & 0.23 & 0.43 & 9 & 1.6 & 99.1 & No \\
\hline 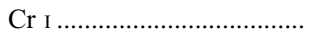 & -0.06 & 0.08 & 0.14 & 0.57 & 18 & 5.3 & 99.7 & No \\
\hline 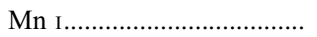 & -0.32 & 0.11 & 0.18 & 0.61 & 18 & 6.8 & 98.6 & No \\
\hline 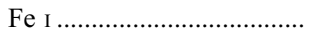 & -1.50 & 0.07 & 0.09 & 0.78 & 25 & 15.6 & 90.1 & No \\
\hline 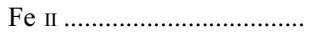 & -1.46 & 0.10 & 0.12 & 0.83 & 21 & 15.1 & 77.1 & No \\
\hline 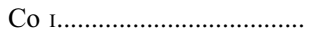 & -0.02 & 0.04 & 0.11 & 0.36 & 8 & 11.5 & 99.2 & No \\
\hline 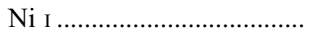 & -0.10 & 0.09 & 0.10 & 0.90 & 24 & 16.1 & 85.2 & No \\
\hline 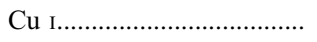 & -0.65 & 0.08 & 0.17 & 0.47 & 14 & 3.2 & 99.7 & No \\
\hline 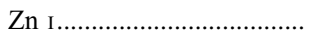 & -0.01 & 0.11 & 0.13 & 0.85 & 18 & 13.0 & 73.9 & No \\
\hline 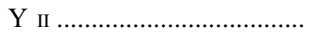 & -0.17 & 0.19 & 0.15 & 1.27 & 18 & 26.6 & 10.1 & No \\
\hline 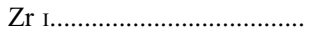 & -0.09 & 0.20 & 0.14 & 1.43 & 4 & 6.2 & 14.4 & No \\
\hline 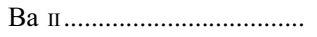 & 0.26 & 0.16 & 0.21 & 0.76 & 25 & 13.7 & 95.4 & No \\
\hline 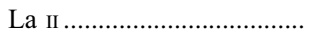 & 0.14 & 0.10 & 0.13 & 0.77 & 13 & 6.8 & 87.2 & No \\
\hline 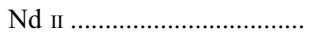 & 0.28 & 0.10 & 0.11 & 0.91 & 14 & 11.5 & 56.9 & No \\
\hline 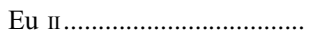 & 0.57 & 0.11 & $\ldots$ & $\ldots$ & 12 & 14.0 & 33.2 & No \\
\hline 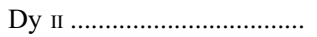 & 0.10 & 0.10 & $\ldots$ & $\ldots$ & 4 & 3.1 & 37.2 & No \\
\hline
\end{tabular}

a The value $\sigma($ tot $)$ is defined in $\S 6.2$.

b This is the ratio of $\sigma$ for the M13 sample stars to $\sigma($ tot $)$. See text.

c The number of stars in the M13 sample in which lines of this species were detected.

${ }^{\mathrm{d}}$ Defined as $\chi^{2}=\sum\left(y_{i}-\bar{y}\right)^{2} / \sigma_{i}^{2}$, where $\bar{y}=\sum\left(y_{i} / \sigma_{i}^{2}\right) / \sum\left(1 / \sigma_{i}^{2}\right)$. A minimum $\sigma_{i}=\sigma($ tot $)$ was adopted.

e The value $p(\chi)$ is the probability of exceeding $\chi^{2}$ by chance.

${ }^{\mathrm{f}}$ If $p(\chi)<0.1 \%$, there is abundance variation; if $p(\chi)>0.5 \%$, there is no variation.

$\mathrm{g}[\mathrm{Al} / \mathrm{Fe}]$ is based on the weak and rarely used line at $5557 \AA$, detected in only three of the most luminous stars of M13.

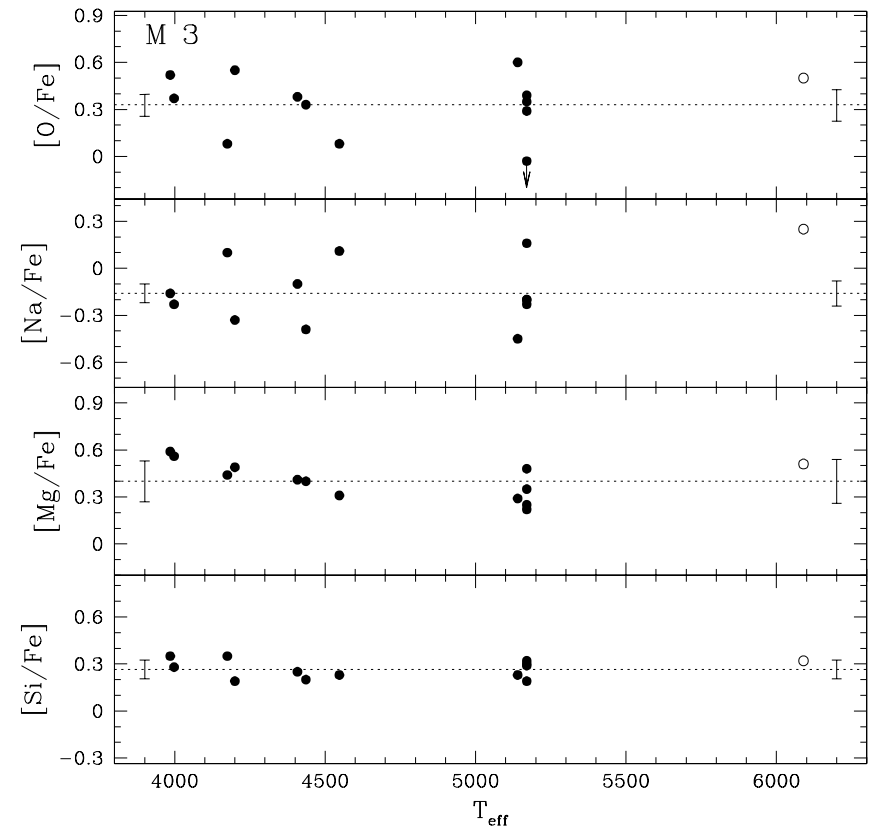

FIG. 4.- $[\mathrm{X} / \mathrm{Fe}]$ for the elements $\mathrm{O}, \mathrm{Na}, \mathrm{Mg}$, and $\mathrm{Si}$ shown as a function of $T_{\text {eff }}$ for our sample of 13 stars in M3. The open circle indicates the HB star. The error bars for the most luminous and least luminous stars, as well as the cluster mean, are indicated as in Fig. 2.

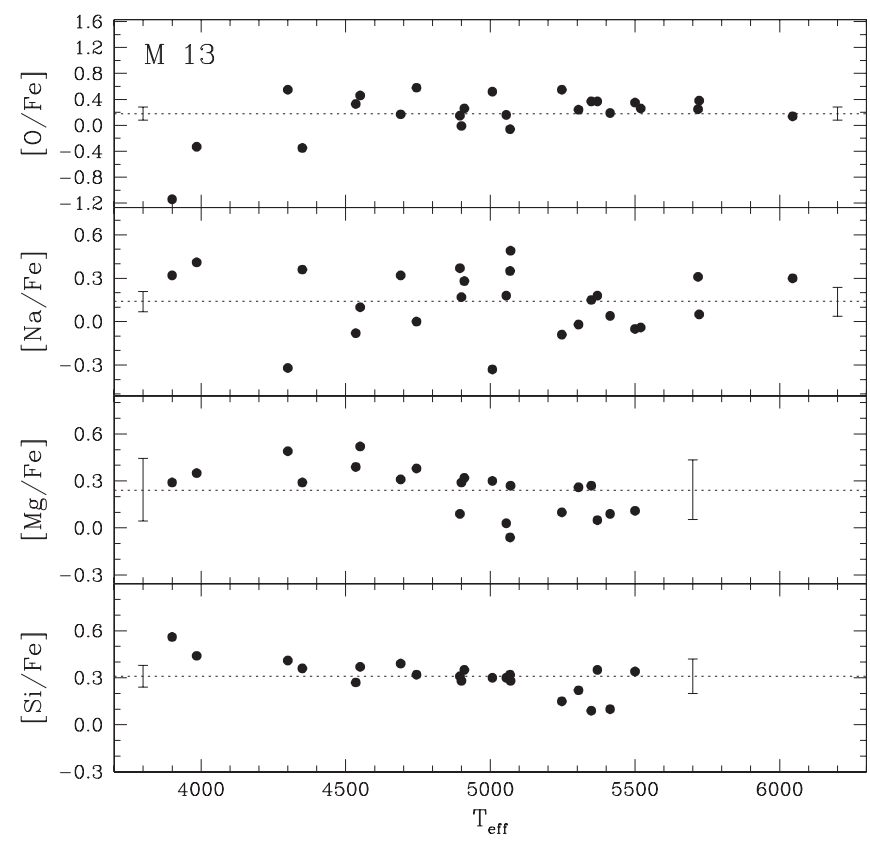

FIG. 5.- $[\mathrm{X} / \mathrm{Fe}]$ for the elements $\mathrm{O}, \mathrm{Na}, \mathrm{Mg}$, and $\mathrm{Si}$ shown as a function of $T_{\text {eff }}$ for our sample of 13 stars in M13. The error bars for the most luminous and least luminous stars, as well as the cluster mean, are indicated as in Fig. 2. 


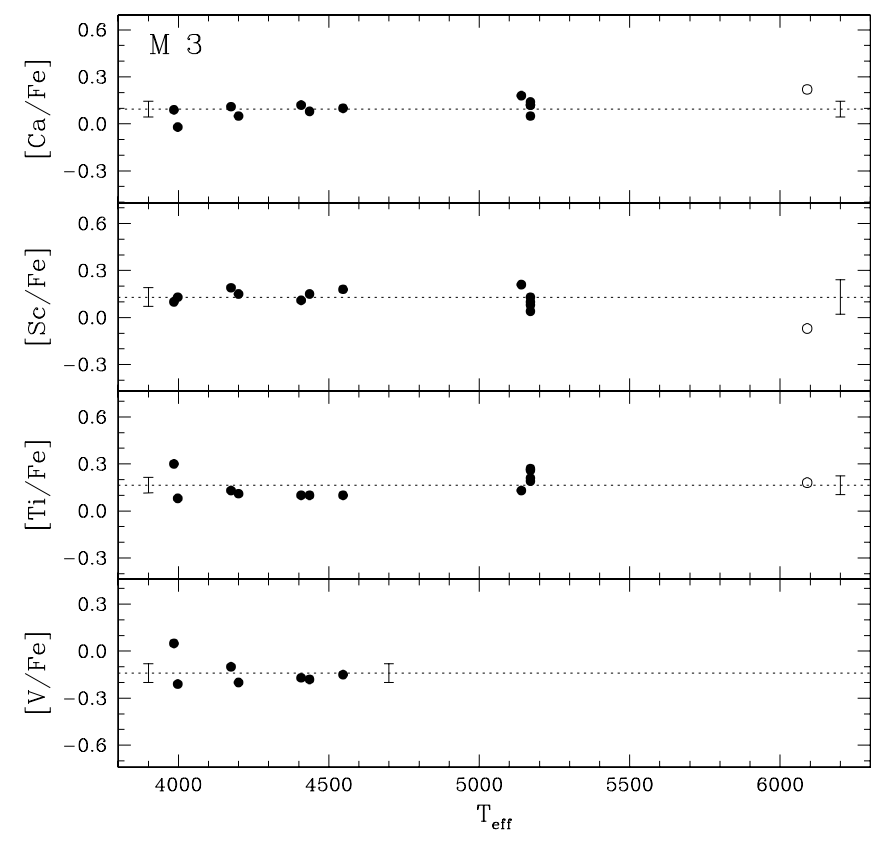

FIG. 6.-Same as Fig. 4, but for the elements $\mathrm{Ca}, \mathrm{Sc}, \mathrm{Ti}$, and V.

be due to difference in the absolute scaling of the Ca I $g f$-values adopted by the Lick-Texas group and by us. This may also play a role for Sc II, but there are not sufficient lines in common to be certain. The HFS corrections for Sc II in these stars are probably not large enough to contribute significantly to this difference.

Because we are interested in star-to-star variations in abundance, we also carry out a comparison of our derived $\mathrm{O}$ and $\mathrm{Na}$ abundances for the individual stars in common with the sample of bright giants of Sneden et al. (2004), rather than comparing the mean of the samples in M3, as was done in Table 12. We choose to compare only with the most recent detailed abundance analysis for stars in these two clusters, ignoring earlier work, in hopes of demonstrating good agreement. The results are shown in Table 13A (Table 13B); the mean differences for each species have been removed, thus removing the systematic

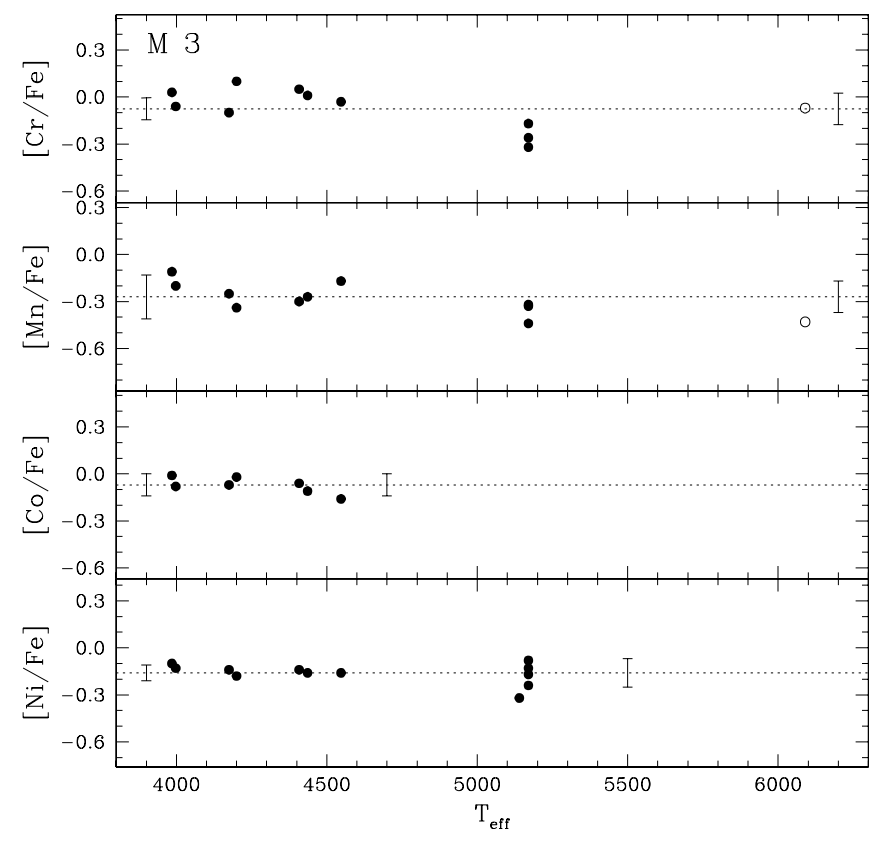

Fig. 8. - Same as Fig. 4, but for the elements Cr, Mn, Co, and Ni.

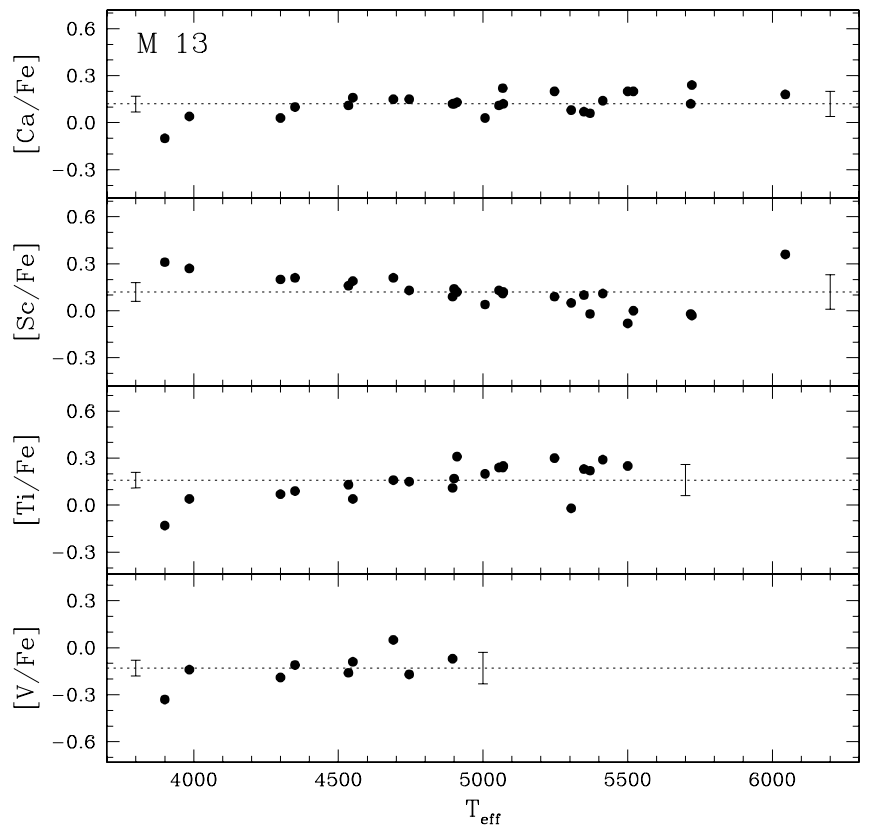

FIG. 7.-Same as Fig. 5, but for the elements $\mathrm{Ca}, \mathrm{Sc}$, Ti, and V.

differences in the analyses. The table reveals the scatter about the mean, i.e., the nonsystematic differences, whose variance is given as the final column in each table. There are three stars in common in M3 and five in M13. For these stars in common, the $\mathrm{Na}$ abundances as analyzed by Sneden et al. (2004) and by us are in extremely good agreement, ignoring a constant offset between us and the Lick-Texas group, but the $\mathrm{O}$ abundances are not. Overall, for M3, the agreement is pretty good, with $\sigma \leq$ 0.15 dex for nine of the 12 species; only for $[\mathrm{O} / \mathrm{Fe}],[\mathrm{Sc} / \mathrm{Fe}]$, and $[\mathrm{Eu} / \mathrm{Fe}]$ is it larger. $\mathrm{O}$ has only a few weak lines in the relevant wavelength region, and difficulties in determining the $\mathrm{O}$ abundance are notorious. $[\mathrm{Eu} / \mathrm{Fe}]$ has essentially the same mean in our analysis of M3 and of M13 as was found by Sneden et al. (2004). There is only one line used by both analyses, that of Eu II at $6645 \AA$, which is very weak. The derived Sc abundances

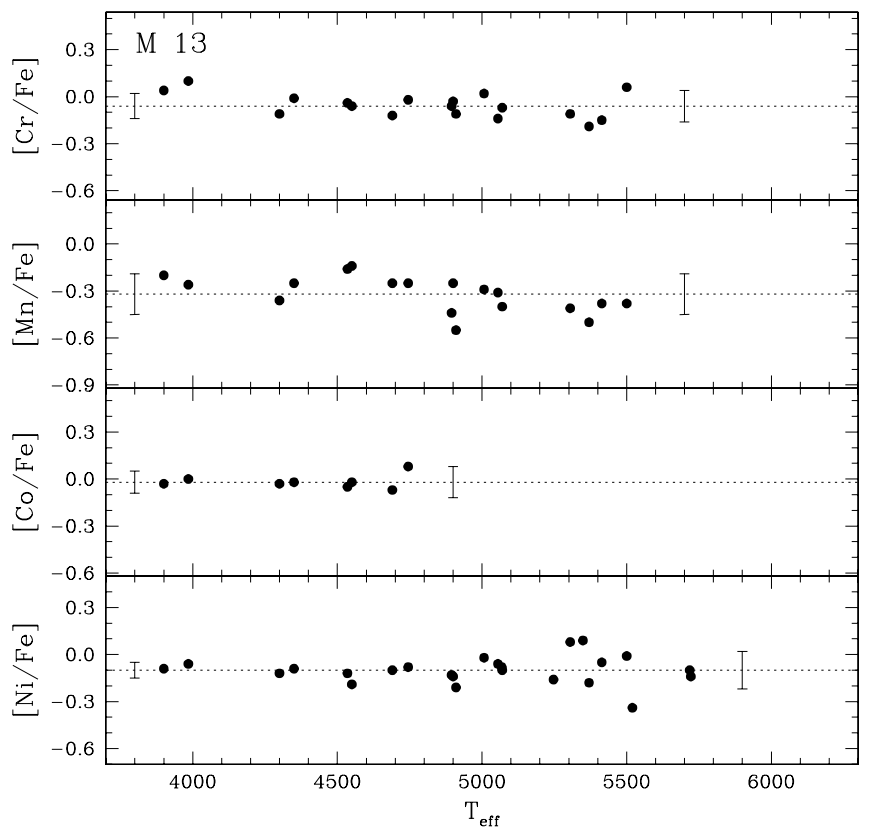

Fig. 9.-Same as Fig. 5, but for the elements Cr, Mn, Co, and Ni. 


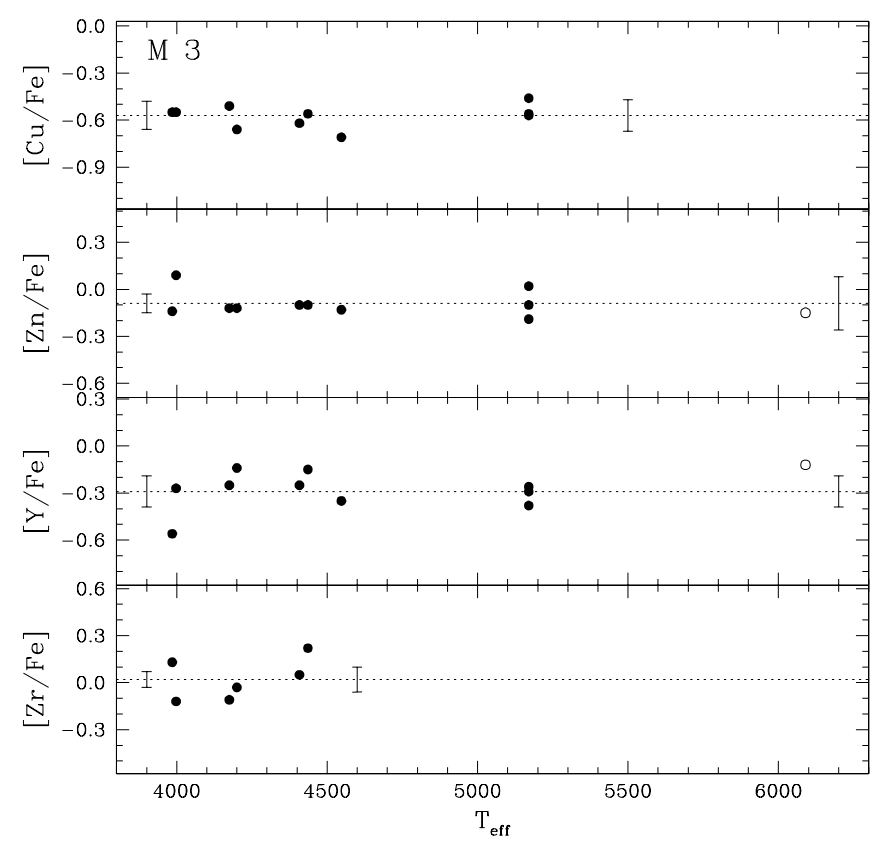

FIG. 10.- Same as Fig. 4, but for the elements $\mathrm{Cu}, \mathrm{Zn}, \mathrm{Y}$, and $\mathrm{Zr}$.

might be affected by differences in the treatment of HFS. The largest $\sigma$ is 0.22 dex, for $[\mathrm{Eu} / \mathrm{Fe}]$. In M13 two of the eight differences have $\sigma>0.3 \operatorname{dex}([\mathrm{O} / \mathrm{Fe}]$ and $[\mathrm{La} / \mathrm{Fe}])$, again two species with only a few weak lines. ( $\mathrm{Sc}$ is not included in Sneden et al.'s analysis for M13, while there are no stars in common in $\mathrm{M} 3$ with $[\mathrm{La} / \mathrm{Fe}]$ measurements.)

Having already removed the systematic differences, one might admit "random" variations in deduced abundance ratios of up to 0.15 dex as resulting from different assumptions made in these two independent analyses. However, the larger differences found for M13, with $\sigma>0.3$ dex for two species, suggest that the abundance errors in one or both of these analyses are being underestimated. We have compared our measured $W_{\lambda}$ with those of the Lick-Texas group, when available. The agree-

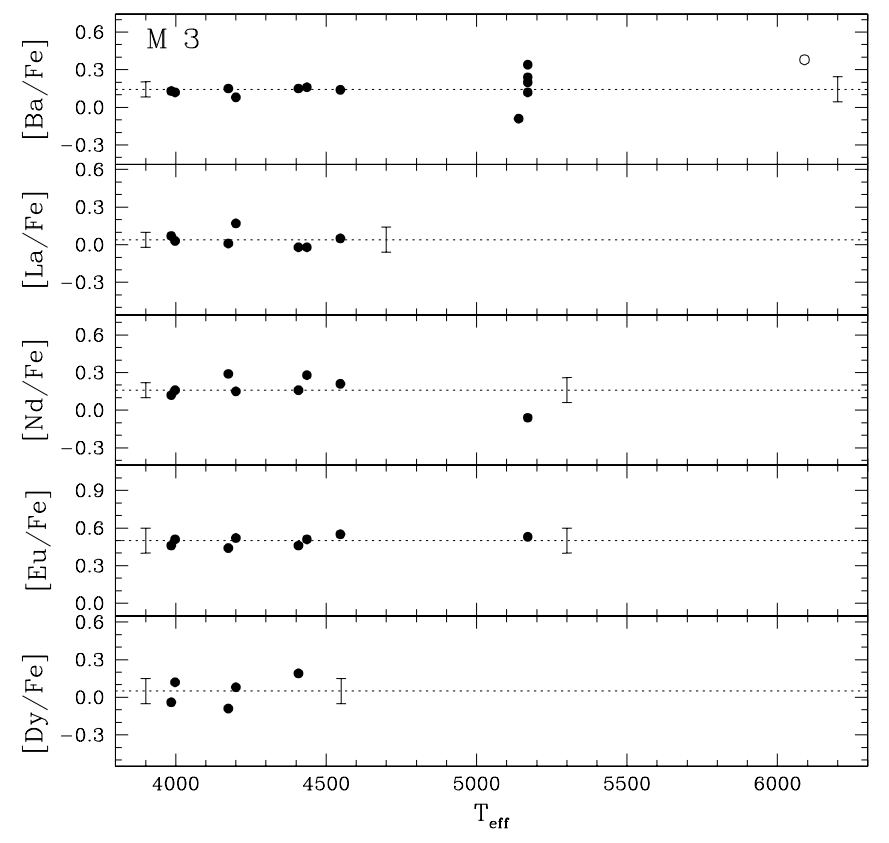

FIG. 12.-Same as Fig. 4, but for the elements $\mathrm{Ba}, \mathrm{La}, \mathrm{Nd}, \mathrm{Eu}$, and $\mathrm{Dy}$.

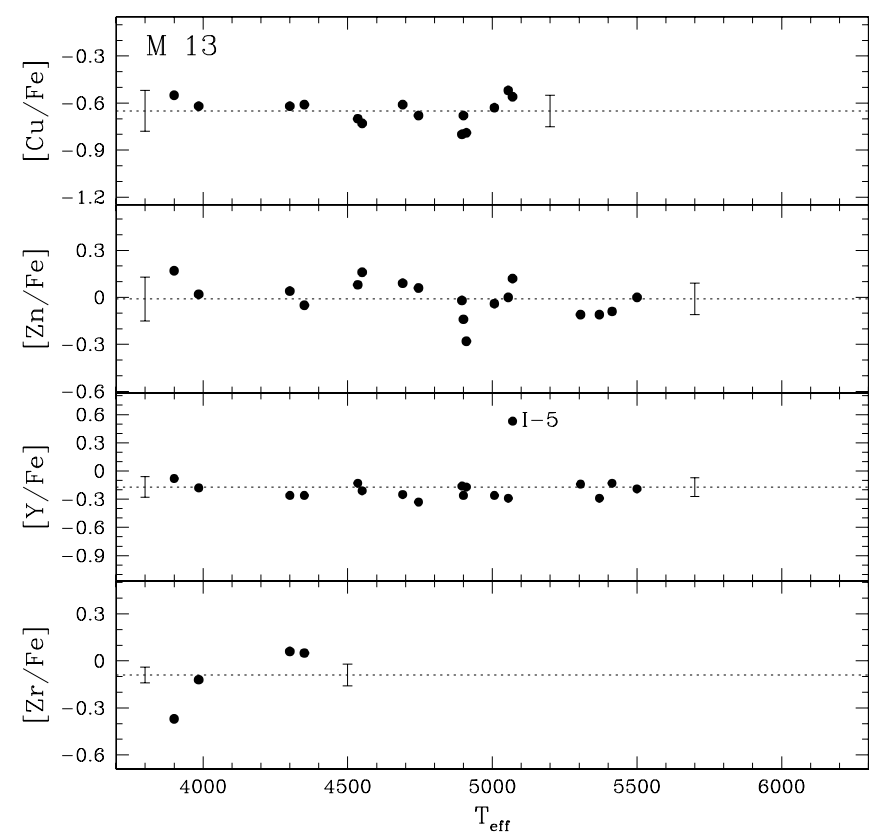

FIG. 11.-Same as Fig. 5, but for the elements $\mathrm{Cu}, \mathrm{Zn}, \mathrm{Y}$, and $\mathrm{Zr}$.

ment is extremely good: $W_{\lambda}$ (our work: Keck) $-W_{\lambda}$ (Sneden et al 2004 , Keck) has a mean of $7 \%$ with $\sigma=7 \%$, while $W_{\lambda}$ (our work: Keck $)-W_{\lambda}$ (Kraft et al 1992, Lick) has a mean of $-5 \%$ with $\sigma=8 \%{ }^{6}$

To summarize, there is very good agreement on a star-by-star basis for the derived $[\mathrm{Na} / \mathrm{Fe}]$ in $\mathrm{M} 3$ and in $\mathrm{M} 13$ for the sample of stars in common with Sneden et al. (2004). The agreement in derived $[\mathrm{O} / \mathrm{Fe}]$ is not as good, but fortunately the star-to-star variations in $\mathrm{O}$ are large. Furthermore, we believe that we understand the origin of most of the discrepancies and that our analysis is sound. Hence, we have some confidence that we may

\footnotetext{
${ }^{6}$ This good agreement suggests that our adoption of the uncertainty in $W_{\lambda}$ as $10 \%$ is reasonable.
}

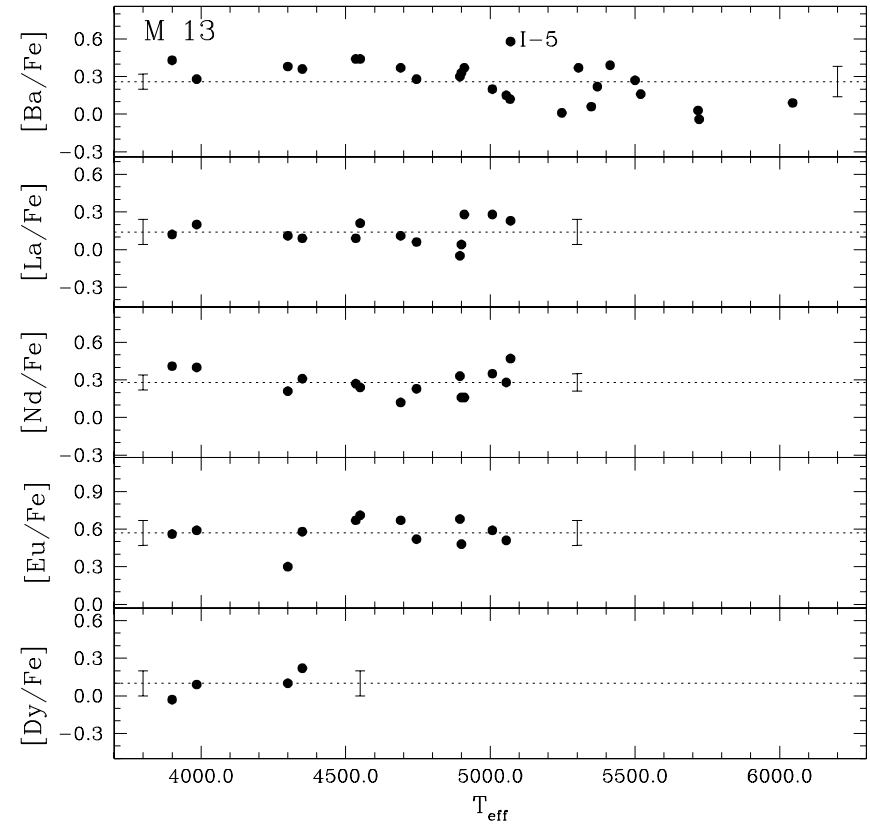

Fig. 13.- Same as Fig. 5, but for the elements Ba, La, Nd, Eu, and Dy. 


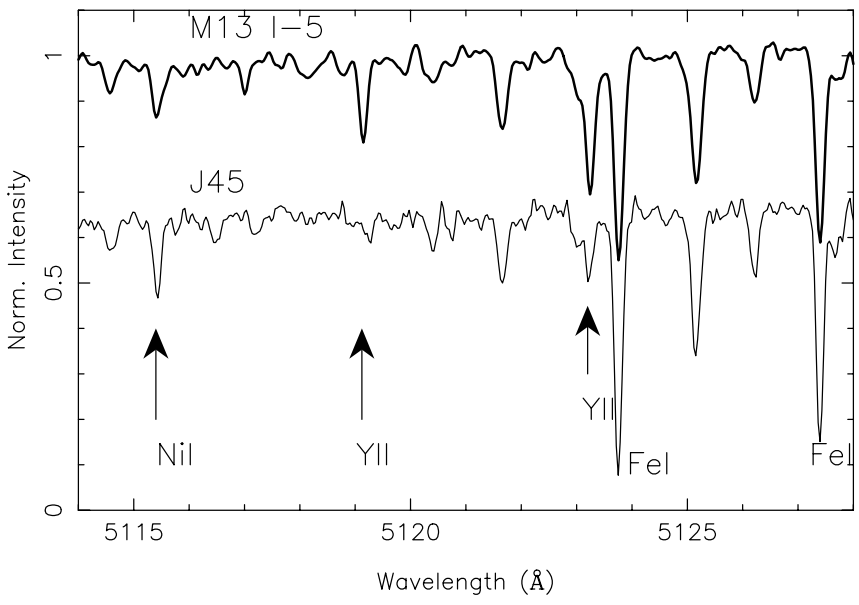

FIG. 14.- Section of the spectrum of the Y-rich star M13 I-5 shown in the region of several Y II lines. The same region in the spectrum of star M13 J45, a star $0.5 \mathrm{mag}$ brighter in $V$ along the RGB, and hence slightly cooler than star I-5, is shown for comparison. proceed to analyze the abundance spreads in terms of star-tostar variations in $[\mathrm{X} / \mathrm{Fe}]$.

\section{CORRELATED ABUNDANCE VARIATIONS OF THE LIGHT ELEMENTS}

$\mathrm{C}, \mathrm{N}, \mathrm{O}, \mathrm{Na}, \mathrm{Mg}$, and $\mathrm{Al}$ are known to show correlated abundance variations from star-to-star among the most luminous stars in globular clusters; see, e.g., the review of Kraft (1994). Our simple SR analysis (see $\S$ 6.2) shows definite starto-star variations in abundance of both $\mathrm{O}$ and $\mathrm{Na}$ in both $\mathrm{M} 3$ and in M13. Variations in $\mathrm{Mg}$, if present, are smaller and subtle. Al is not effectively covered in our spectra. ${ }^{7}$

It is well established that $\mathrm{O}$ and $\mathrm{Na}$ are anticorrelated among luminous giants in globular clusters; see, e.g., Kraft (1994). Furthermore, Ramírez \& Cohen (2002) compiled the data from the literature, combined it with their own, and showed that the same linear relation can be used to fit the $\mathrm{O}$ and $\mathrm{Na}$ data for all globular clusters studied in detail thus far. The latest addition to the clusters studied in detail, NGC 2808, by Carretta et al. (2004a), does so as well. The question of interest is what happens when we look at lower luminosity stars.

Figure 15 shows the relationship between $\mathrm{Na}$ and $\mathrm{O}$ abundances (both with respect to Fe) for our sample in M3. Also superposed is the line representing the fit for this anticorrelation determined by Sneden et al. (2004) for the luminous giants in

\footnotetext{
${ }^{7}$ Our spectra only include the line at $5557 \AA$, which is very weak and only detected in a few stars; they do not include the most commonly used Al lines.
}

TABLE 11

Comparison with Atmospheric Parameters from the Literature

\begin{tabular}{|c|c|c|c|c|c|}
\hline \multirow[b]{2}{*}{ ID } & \multicolumn{2}{|c|}{ THIS WORK } & \multicolumn{3}{|r|}{ LITERATURE } \\
\hline & $T_{\text {eff }}$ & $\log g$ & $T_{\text {eff }}$ & $\log g$ & Reference \\
\hline \multicolumn{6}{|c|}{ M3 } \\
\hline \multirow[t]{4}{*}{ 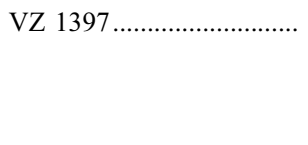 } & 3985 & 0.40 & 3950 & 0.40 & Sneden et al. (2004), photometric \\
\hline & & & 3925 & 0.10 & Sneden et al. (2004), spectroscopic \\
\hline & & & 3950 & 0.40 & Kraft et al. (1992) \\
\hline & & & 4000 & 0.6 & Cohen (1978) \\
\hline \multirow[t]{3}{*}{ II-46 .............................. } & 3998 & 0.40 & 4050 & 0.40 & Sneden et al. (2004) \\
\hline & & & 4000 & 0.60 & Kraft et al. (1992) \\
\hline & & & 4000 & 0.6 & Cohen (1978) \\
\hline \multirow[t]{3}{*}{ 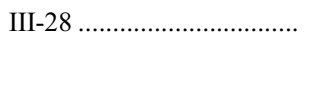 } & 4200 & 0.60 & 4175 & 0.55 & Cavallo \& Nagar (2000) \\
\hline & & & 4160 & 0.75 & Kraft et al. (1992) \\
\hline & & & 4100 & 0.7 & Cohen (1978) \\
\hline \multirow[t]{3}{*}{ 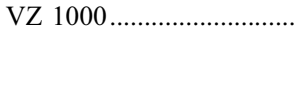 } & 4175 & 0.70 & 4125 & 0.60 & Sneden et al. (2004) \\
\hline & & & 4200 & 0.65 & Cavallo \& Nagar (2000) \\
\hline & & & 4175 & 0.45 & Kraft et al. (1992) \\
\hline \multicolumn{6}{|c|}{ M13 } \\
\hline \multirow[t]{3}{*}{ 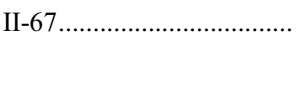 } & 3900 & 0.45 & 3900 & 0.37 & Sneden et al. (2004) \\
\hline & & & 3950 & 0.20 & Kraft et al. (1997) \\
\hline & & & 3950 & 0.30 & Kraft et al. (1992) \\
\hline \multirow[t]{4}{*}{ 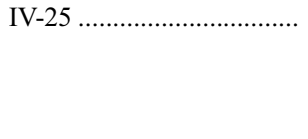 } & 3985 & 0.50 & 3975 & 0.38 & Sneden et al. (2004) \\
\hline & & & 4000 & 0.15 & Kraft et al. (1997) \\
\hline & & & 4000 & 0.30 & Kraft et al. (1992) \\
\hline & & & 4000 & 0.5 & Cohen (1978) \\
\hline \multirow[t]{2}{*}{ 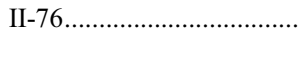 } & 4300 & 0.85 & 4285 & 0.80 & Sneden et al. (2004) \\
\hline & & & 4350 & 1.00 & Kraft et al. (1992) \\
\hline \multirow[t]{2}{*}{ 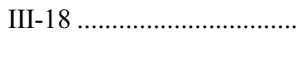 } & 4350 & 1.00 & 4330 & 0.95 & Sneden et al. (2004) \\
\hline & & & 4350 & 1.20 & Kraft et al. (1992) \\
\hline \multirow[t]{2}{*}{ 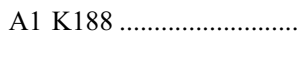 } & 4535 & 1.35 & 4550 & 1.34 & Sneden et al. (2004) \\
\hline & & & 4550 & 1.50 & Kraft et al. (1997) \\
\hline
\end{tabular}


TABLE 12

Comparison of Deduced Abundance Ratios with Sneden et al. (2004)

\begin{tabular}{|c|c|c|c|c|c|}
\hline \multirow[b]{2}{*}{ SPECIES } & \multicolumn{2}{|c|}{ THIS WORK } & \multicolumn{2}{|c|}{ SNEDEN ET AL. (2004) } & \multirow{2}{*}{$\frac{\text { OUR WORK }- \text { SNEDEN }}{\Delta([\mathrm{X} / \mathrm{Fe}])(\mathrm{dex})}$} \\
\hline & {$[\mathrm{X} / \mathrm{Fe}](\mathrm{dex})$} & $\sigma_{N}^{\mathrm{a}}(\mathrm{dex})$ & {$[\mathrm{X} / \mathrm{Fe}](\mathrm{dex})$} & $\sigma_{N}^{\mathrm{a}}(\mathrm{dex})$ & \\
\hline \multicolumn{6}{|c|}{ M3 } \\
\hline $\mathrm{O}_{\text {I }}$ & +0.33 & 0.05 & +0.15 & 0.03 & +0.18 \\
\hline 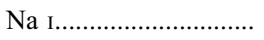 & -0.16 & 0.06 & +0.01 & 0.04 & -0.17 \\
\hline Mg I................................ & +0.40 & 0.03 & +0.22 & 0.03 & +0.18 \\
\hline $\mathrm{Si}$ I ................................... & +0.27 & 0.02 & +0.30 & 0.01 & -0.03 \\
\hline 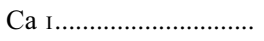 & +0.10 & 0.01 & +0.23 & 0.01 & -0.13 \\
\hline 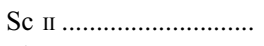 & +0.13 & 0.01 & -0.11 & 0.02 & +0.24 \\
\hline Ti I............................... & +0.17 & 0.02 & +0.21 & 0.01 & -0.04 \\
\hline 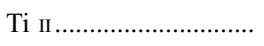 & +0.27 & 0.03 & +0.16 & 0.02 & +0.11 \\
\hline V I & -0.14 & 0.03 & -0.04 & 0.02 & -0.10 \\
\hline 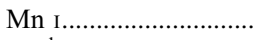 & -0.27 & 0.03 & -0.37 & 0.01 & +0.10 \\
\hline $\mathrm{Fe}_{\mathrm{I}}^{\mathrm{b}}$ & -1.39 & 0.02 & -1.62 & 0.01 & +0.23 \\
\hline $\mathrm{Fe}$ II $^{\mathrm{b}}$ & -1.43 & 0.02 & -1.55 & 0.02 & +0.12 \\
\hline 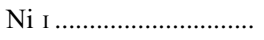 & -0.16 & 0.02 & -0.05 & 0.01 & -0.11 \\
\hline 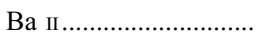 & +0.15 & 0.03 & +0.21 & 0.02 & -0.06 \\
\hline 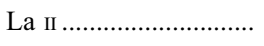 & +0.04 & 0.02 & +0.09 & 0.02 & -0.05 \\
\hline 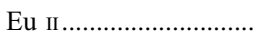 & +0.50 & 0.01 & +0.54 & 0.03 & -0.04 \\
\hline \multicolumn{6}{|c|}{ M13 } \\
\hline $\mathrm{O}_{\text {I }}$ & +0.18 & 0.08 & -0.13 & 0.06 & +0.31 \\
\hline 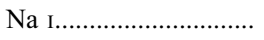 & +0.14 & 0.04 & +0.21 & 0.04 & -0.07 \\
\hline 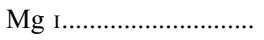 & +0.24 & 0.03 & +0.11 & 0.03 & +0.21 \\
\hline $\mathrm{Al}$ I & +0.74 & 0.06 & +0.75 & 0.11 & -0.01 \\
\hline $\mathrm{Fe}_{\mathrm{I}}^{\mathrm{b}} \ldots \ldots \ldots \ldots$ & -1.50 & 0.01 & -1.63 & 0.03 & +0.13 \\
\hline $\mathrm{Fe}$ II $^{\mathrm{b}}$ & -1.46 & 0.02 & -1.49 & 0.06 & +0.03 \\
\hline Ва ІІ ................................... & +0.26 & 0.03 & +0.24 & 0.03 & +0.02 \\
\hline La II ................................... & +0.14 & 0.03 & +0.11 & 0.03 & +0.03 \\
\hline 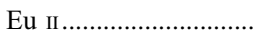 & +0.57 & 0.03 & +0.49 & 0.03 & +0.08 \\
\hline
\end{tabular}

${ }^{\text {a }}$ The value $\sigma_{N}$ is the uncertainty in the mean value of $[\mathrm{X} / \mathrm{Fe}]$ for the cluster, i.e., $\sigma / \sqrt{N_{\text {stars }}}$.

b These values are $[\mathrm{Fe} / \mathrm{H}]$.

M13, shifted by +0.07 dex in $[\mathrm{O} / \mathrm{Fe}]$. The first and last quartiles of the O-Na anticorrelation seen by Sneden et al. (2004) in their sample of luminous giants in M3 are indicated. There is a reasonably clear anticorrelation even for the faintest stars in our M3 sample, which agrees well with that of Sneden et al. (2004), given a small shift in $\mathrm{O}$ abundance scale. Thus, the anticor-

TABLE 13A

Star-By-STAR Comparison of AbUndances for M3 ${ }^{\text {a }}$

\begin{tabular}{|c|c|c|c|c|}
\hline Species & VZ 1397 & II- 46 & VZ 1000 & $\sigma$ \\
\hline$[\mathrm{O} / \mathrm{Fe}] \ldots \ldots \ldots \ldots \ldots \ldots$ & 0.21 & -0.13 & 0.02 & 0.17 \\
\hline$[\mathrm{Na} / \mathrm{Fe}] \ldots \ldots \ldots \ldots \ldots$ & 0.09 & 0.14 & 0.17 & 0.04 \\
\hline$[\mathrm{Mg} / \mathrm{Fe}] \ldots \ldots \ldots \ldots . .$. & 0.09 & $\ldots$ & $\ldots$ & $\ldots$ \\
\hline$[\mathrm{Si} / \mathrm{Fe}] \ldots \ldots \ldots \ldots \ldots$ & 0.03 & 0.10 & 0.09 & 0.04 \\
\hline$[\mathrm{Ca} / \mathrm{Fe}] \ldots \ldots \ldots \ldots \ldots$ & -0.04 & -0.04 & 0.01 & 0.03 \\
\hline$[\mathrm{Sc} / \mathrm{Fe}] \ldots \ldots \ldots \ldots \ldots$ & -0.12 & 0.09 & 0.19 & 0.16 \\
\hline$[\mathrm{Ti} / \mathrm{Fe}] \ldots \ldots \ldots \ldots \ldots$ & 0.03 & -0.09 & 0.05 & 0.08 \\
\hline$[\mathrm{V} / \mathrm{Fe}] \ldots \ldots \ldots \ldots \ldots \ldots$ & 0.15 & -0.10 & -0.04 & 0.13 \\
\hline$[\mathrm{Mn} / \mathrm{Fe}] \ldots \ldots \ldots \ldots \ldots$ & 0.09 & 0.10 & -0.05 & 0.08 \\
\hline$[\mathrm{Fe} / \mathrm{H}] \ldots \ldots \ldots \ldots \ldots$ & -0.06 & -0.16 & -0.08 & 0.05 \\
\hline 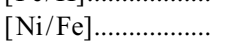 & 0.08 & 0.12 & 0.00 & 0.06 \\
\hline$[\mathrm{Ba} / \mathrm{Fe}] \ldots \ldots \ldots \ldots \ldots$ & 0.21 & 0.24 & 0.15 & 0.05 \\
\hline$[\mathrm{La} / \mathrm{Fe}] \ldots \ldots \ldots \ldots \ldots$ & 0.16 & $\ldots$ & $\ldots$ & $\ldots$ \\
\hline$[\mathrm{Eu} / \mathrm{Fe}] \ldots \ldots \ldots \ldots \ldots$ & 0.07 & 0.24 & -0.20 & 0.22 \\
\hline
\end{tabular}

${ }^{\mathrm{a}}[\mathrm{X} / \mathrm{Fe}]($ this work $)-[\mathrm{X} / \mathrm{Fe}]($ Sneden et al. 2004) $-($ mean $\Delta$ from Table 12). relation between $\mathrm{O}$ and $\mathrm{Na}$ persists in $\mathrm{M} 3$ from the RGB to at least $V=16.6 \mathrm{mag}$, which is about $2 \mathrm{mag}$ fainter than the regime over which Sneden et al. (2004) established this relationship. The anticorrelation between $\mathrm{O}$ and $\mathrm{Na}$ has approximately the same slope and extends over approximately the same range among the fainter stars in M3, as it does among the most luminous M3 giants.

Although Sneden et al. (2004) claim a marginal detection of variations in $[\mathrm{Mg} / \mathrm{Fe}]$ among their sample of luminous giants in $\mathrm{M}$, with increasing $\mathrm{Mg}$ abundance being correlated with increasing $\mathrm{Na}$ abundance, we fail to find any credible evidence of such in M3; it might be only slightly larger than $\sigma$ (tot).

TABLE 13B

Star-by-Star Comparison of Abundances for M13 ${ }^{\mathrm{a}}$

\begin{tabular}{crrcccc}
\hline \hline Species & II-67 & IV-25 & II-76 & III-18 & K188 & $\sigma$ \\
\hline$[\mathrm{O} / \mathrm{Fe}] \ldots \ldots \ldots \ldots .$. & 0.17 & 0.44 & -0.06 & -0.40 & -0.28 & 0.34 \\
{$[\mathrm{Na} / \mathrm{Fe}] \ldots \ldots \ldots$.} & -0.03 & -0.04 & 0.02 & -0.01 & -0.02 & 0.02 \\
{$[\mathrm{Mg} / \mathrm{Fe}] \ldots \ldots \ldots$.} & 0.06 & 0.06 & $\ldots$ & $\ldots$ & -0.16 & 0.13 \\
{$[\mathrm{Al} / \mathrm{Fe}] \ldots \ldots \ldots .$.} & -0.51 & -0.35 & $\ldots$ & $\ldots$ & $\ldots$ & 0.11 \\
{$[\mathrm{Fe} / \mathrm{H}] \ldots \ldots \ldots$.} & 0.05 & 0.04 & -0.04 & -0.04 & 0.14 & 0.07 \\
{$[\mathrm{Ba} / \mathrm{Fe}] \ldots \ldots \ldots$.} & 0.29 & 0.14 & $\ldots$ & $\ldots$ & 0.14 & 0.09 \\
{$[\mathrm{La} / \mathrm{Fe}] \ldots \ldots \ldots$.} & 0.20 & 0.30 & $\ldots$ & $\ldots$ & -0.32 & 0.33 \\
{$[\mathrm{Eu} / \mathrm{Fe}] \ldots \ldots \ldots$.} & 0.09 & 0.17 & $\ldots$ & $\ldots$ & -0.10 & 0.14 \\
\hline
\end{tabular}

${ }^{\mathrm{a}}[\mathrm{X} / \mathrm{Fe}]($ this work $)-[\mathrm{X} / \mathrm{Fe}]($ Sneden et al. 2004) $-($ mean $\Delta$ from Table 12). 


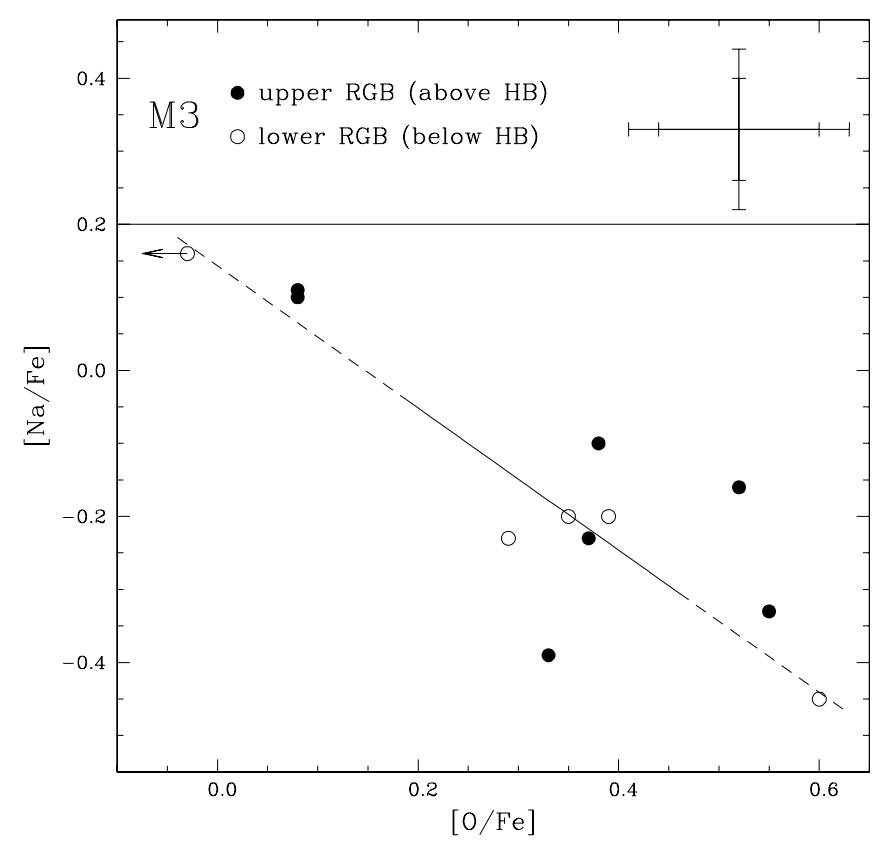

FIG. 15.-Ratio $[\mathrm{Na} / \mathrm{Fe}]$ shown as a function of $[\mathrm{O} / \mathrm{Fe}]$ for our sample of 13 stars in M3. The filled circles denote the luminous RGB stars, the open circles the lower luminosity giants. The error bars typical of the most luminous and least luminous stars in our sample are indicated. The line represents the relationship found by Sneden et al. (2004), with a shift of +0.07 dex in $[O / F e]$ applied; the line is solid between the first and third quartiles of his sample and is dashed outside that regime.

For M13, there is a much richer phenomenology. Figure 16 shows the relationship between $\mathrm{Na}$ and $\mathrm{O}$ abundances (both with respect to $\mathrm{Fe}$ ) for our sample. Also superposed is the line representing the fit for this anticorrelation we determined for the luminous giants in M3, shifted vertically by +0.22 dex. The anticorrelation is

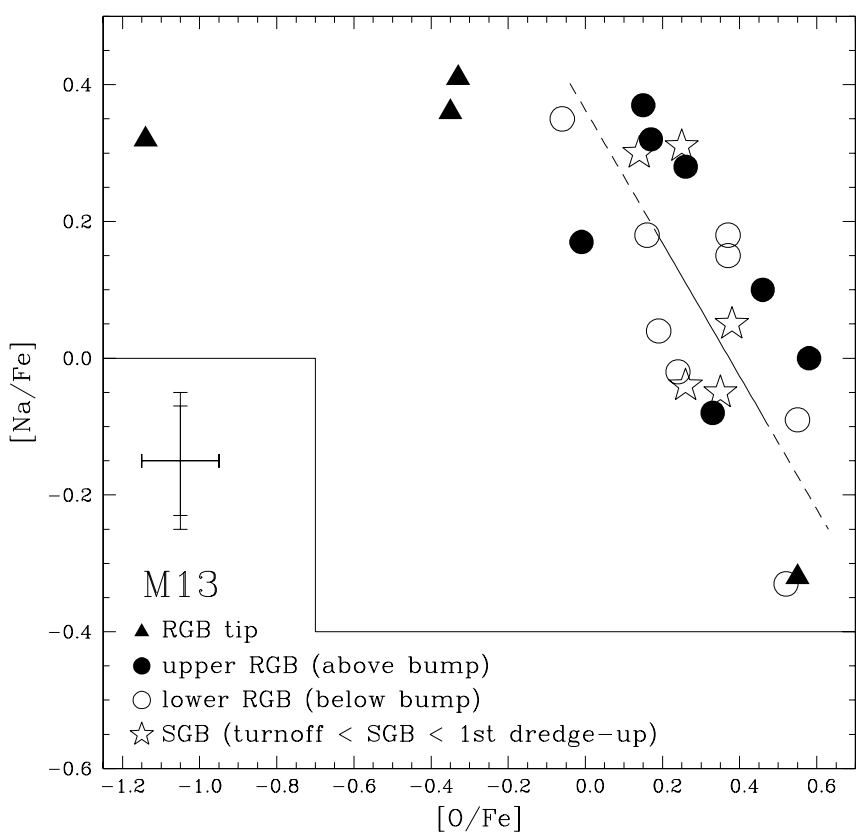

Fig. 16. - Ratio $[\mathrm{Na} / \mathrm{Fe}]$ shown as a function of $[\mathrm{O} / \mathrm{Fe}]$ for 24 of our sample of 25 stars in M13. The error bars typical of the most luminous and least luminous stars in our sample are indicated. The line represents the relationship found for M3, with a vertical offset of +0.22 dex; the line extends over the range covered by the sample of Sneden et al. (2004); it is solid between the first and third quartiles of their sample and dashed outside that regime. The different symbols denote the luminosity of the star.

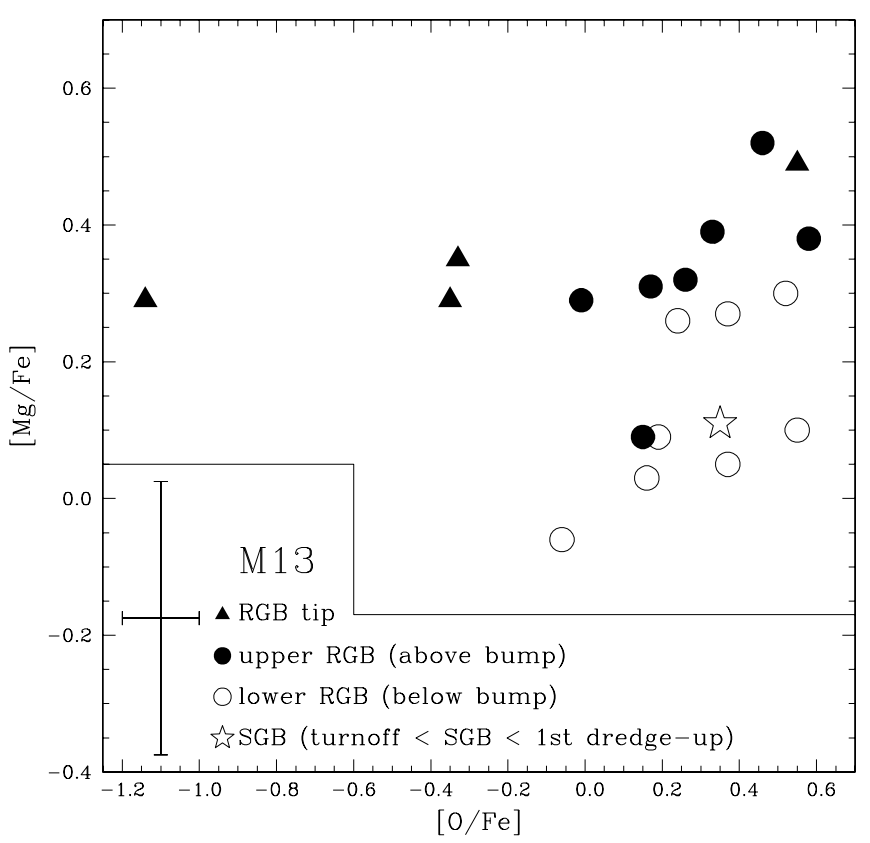

Fig. 17.- Ratio $[\mathrm{Mg} / \mathrm{Fe}]$ shown as a function of $[\mathrm{O} / \mathrm{Fe}]$ for 20 of our sample of 25 stars in M13. The different symbols denote the luminosity of the star. The error bars typical of the most luminous and least luminous stars in our sample are indicated.

immediately apparent. It contains a regime extending to very strong depletion of $\mathrm{O}$ at the highest values of $[\mathrm{Na} / \mathrm{Fe}]$, which is not seen in M3 but was seen by Kraft et al. (1997) in M13. This regime is populated only by the most luminous giants in M13. It is also immediately apparent that the anticorrelation holds to the lowest luminosities probed in the present data set, i.e., to the regime just above the main-sequence turnoff in M13. With the exception of the extremely O-depleted RGB tip stars, the amplitude of the Na-O anticorrelation in M13 appears to be constant over the full range of luminosities in the present sample.

Figure 17 shows the behavior of $\mathrm{Mg}$ as a function of $[\mathrm{O} / \mathrm{Fe}]$. The general previously observed small trend of increasing $\mathrm{Mg}$ as $\mathrm{O}$ increases or $\mathrm{Na}$ decreases is present with an amplitude of $\sim 0.4$ dex, as was found by Kraft et al. (1997). This trend is present at all luminosities probed. However, the mean abundance ratio $[\mathrm{Mg} / \mathrm{Fe}]$ appears to decrease with decreasing luminosity along the RGB. The effect is small, $\sim 0.2$ dex in $[\mathrm{Mg} / \mathrm{Fe}]$ over the range of our sample, but is clearly seen in this figure and also in Figure 18, which shows $[\mathrm{Mg} / \mathrm{Fe}]$ as a function of $[\mathrm{Na} / \mathrm{Fe}]$. A similar difference between $[\mathrm{Mg} / \mathrm{Fe}]$ for dwarfs and for subgiants for stars in metal-poor GCs was noted by Gratton et al. (2001). We are using the same three Mg lines for each star (the $\mathrm{Mg}$ triplet lines are not used), and in all but the three faintest stars all three lines are detected. We use the same atomic parameters throughout. Given the strong increase in $\mathrm{Mg}$ I line strength with luminosity along the RGB, a systematic error in the choice of $v_{t}$ with $T_{\text {eff }}$ could in principle produce this increase in $\mathrm{Mg}$ abundance with luminosity along the M13 RGB. However, a more likely culprit is non-LTE effects in Mg. These, as calculated by Zhao \& Gehren (2000; see also Gehren et al. 2004), increase with $T_{\text {eff }}$ and with decreasing $[\mathrm{Fe} / \mathrm{H}]$ and have the right amplitude and sign to produce this effect.

\subsection{Correlations of $\mathrm{C}, \mathrm{N}$, and $\mathrm{O}$}

Correlated $\mathrm{C}$ and $\mathrm{N}$ variations in M13 have been studied in detail by Briley et al. (2002, 2004). They determined 


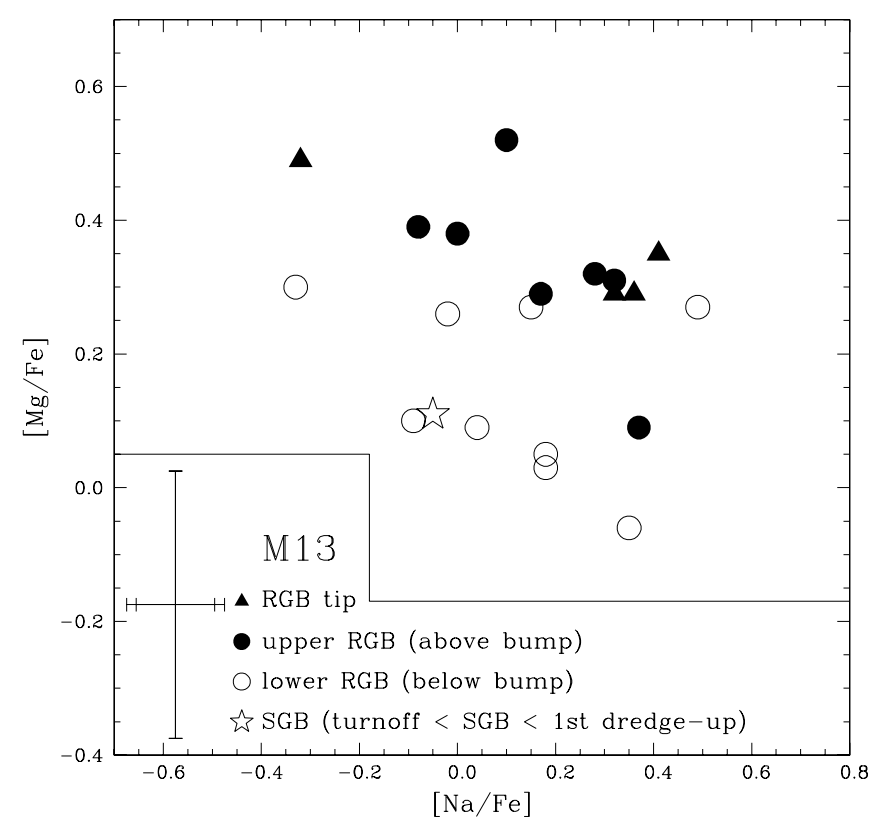

Fig. 18. - Ratio $[\mathrm{Mg} / \mathrm{Fe}]$ shown as a function of $[\mathrm{Na} / \mathrm{Fe}]$ for 21 of our sample of 25 stars in M13. The different symbols denote the luminosity of the star. The error bars typical of the most luminous and least luminous stars in our sample are indicated.

$\mathrm{C}$ abundances from the $\mathrm{G}$ band of $\mathrm{CH}$ for a large sample of stars reaching from the RGB to below the main-sequence turnoff in M13. Combining their results with the earlier work of Smith et al. (1996) and Suntzeff (1981), who cover the most luminous RGB stars in M13, they evaluated the variation from star to star of $[\mathrm{C} / \mathrm{Fe}]$ from the $\mathrm{RGB}$ tip to very low luminosities. They find big spreads in $[\mathrm{C} / \mathrm{Fe}]$ at all luminosities probed, with $[\mathrm{C} / \mathrm{Fe}]$ ranging from -0.8 to -0.2 dex among the lower luminosity giants. There is a marked decline in $[\mathrm{C} / \mathrm{Fe}]$ toward higher luminosities among the upper RGB M13 stars, found originally by Suntzeff (1981). Briley et al. also see a large range in $[\mathrm{N} / \mathrm{Fe}]$ of 0.0 to +1.5 dex.

A crucial test is to determine whether just $\mathrm{CN}$ burning is occurring, $\mathrm{C}$ being transformed into $\mathrm{N}$, in which case $\epsilon(\mathrm{C}+\mathrm{N})$ will be constant, or whether ON burning is also occurring. In either case, the sum $\epsilon(\mathrm{C}+\mathrm{N}+\mathrm{O})$ should be approximately constant, as nucleosynthesis of even heavier elements proceeds only at very high temperatures. Such a test was carried out by Brown et al. (1991) for a small sample of stars; we carry it out for a considerably larger sample of five (12) stars using our new $\mathrm{O}$ abundances and the $\mathrm{C}$ and $\mathrm{N}$ abundances of Smith et al. (1996) or Suntzeff (1981). Figure 19 shows the result for M13; $\log [\epsilon(\mathrm{C}+\mathrm{N}+\mathrm{O})]$ is constant at -1.24 dex with a relatively small $\sigma$ of 0.12 dex; this value is $\sim 0.3$ dex higher than $[\mathrm{Fe} / \mathrm{H}]$ for M13. The sum of $\mathrm{C}+\mathrm{N}$ is not constant, and $\mathrm{O}$ burning, especially near the RGB tip of M13, is required. For M3, the necessary data exists for five stars, combining our new determination of $[\mathrm{O} / \mathrm{Fe}]$ with the $\mathrm{C}$ and $\mathrm{N}$ measurements of Smith et al. (1996), Suntzeff (1981), or of Bell \& Dickens (1980). We find a value of -1.2 dex for the mean of sum of $\log [\epsilon(\mathrm{C}+\mathrm{N}+\mathrm{O})]$, identical to that of M13.

\subsection{Comments on Nucleosynthesis}

We now turn to what we can learn about the chemical history of the two globular clusters M3 and M13 from our work. Figure 20 shows the abundances $[\mathrm{X} / \mathrm{Fe}]$ we have derived for these two populous Galactic GCs. The light elements are

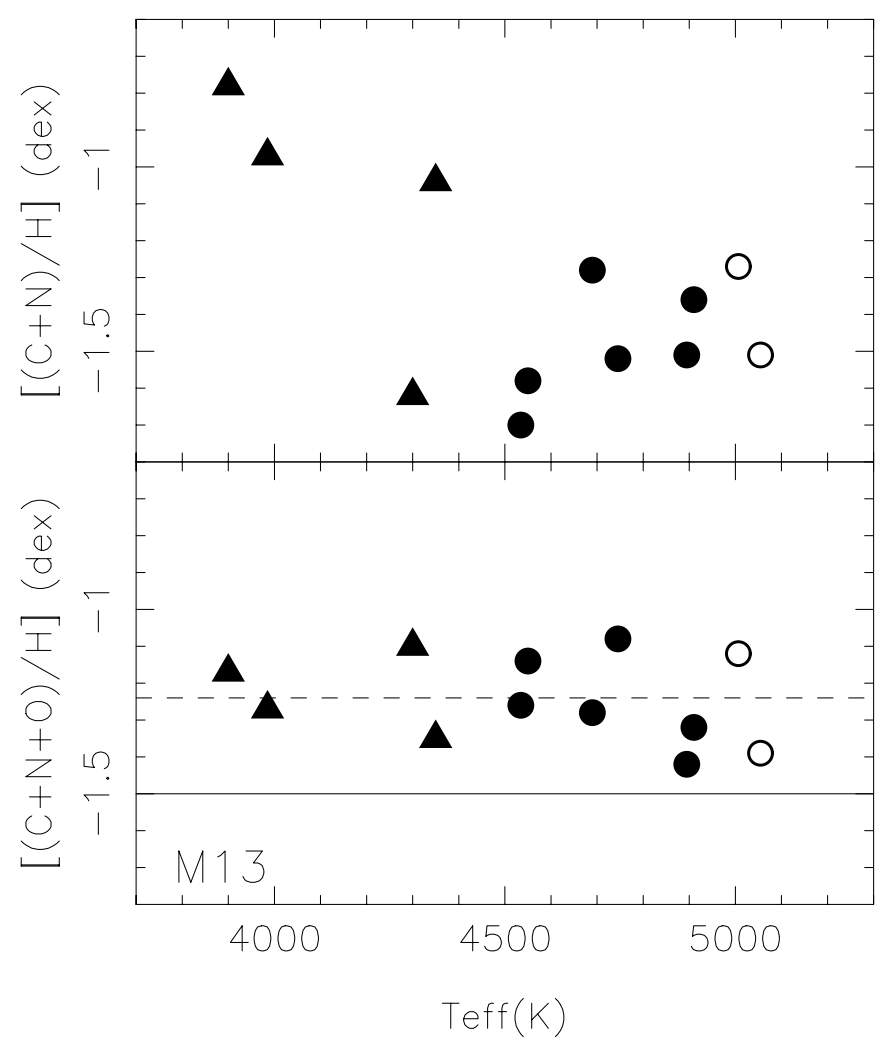

FIG. 19.- - Sum of $\mathrm{C}+\mathrm{N}+\mathrm{O}$ (bottom) and of $\mathrm{C}+\mathrm{N}($ top $)$ shown as a function of $T_{\text {eff }}$ for 12 stars in M13. The different symbols denote the luminosity of the star (as in Fig. 16). The dashed horizontal line in the bottom panel is the mean of $(\mathrm{C}+\mathrm{N}+\mathrm{O}) / \mathrm{H}$, while the solid horizontal line is the cluster mean for $[\mathrm{Fe} / \mathrm{H}]$.

characterized by very large ranges of star-to-star abundance variations. Since the odd atomic number elements among the light elements normally are of lower abundance (in terms of $\mathrm{X} / \mathrm{H}$ ), a small increment of these elements can lead to large enhancements in $[\mathrm{X} / \mathrm{Fe}]$. Such behavior is seen throughout this

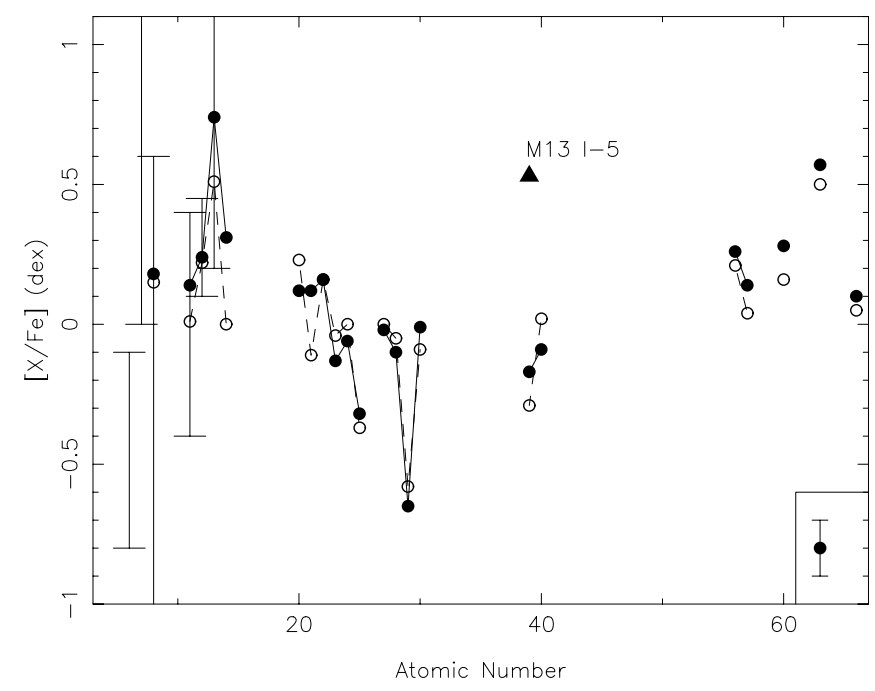

FIG. 20.-Abundance ratios $[\mathrm{X} / \mathrm{Fe}]$ shown as a function of atomic number for M3 and M13. Filled circles are used for M13 and open circles for M3, while a filled triangle denotes $[\mathrm{Y} / \mathrm{Fe}]$ for the peculiar star M13 I-5. Elements with consecutive atomic numbers whose abundances have been determined are indicated by solid (dashed) lines. For those elements which show star-to-star variation, ranges are indicated for $\mathrm{C}, \mathrm{N}$ (both from Briley et al. 2004), $\mathrm{O}, \mathrm{Na}$, $\mathrm{Mg}$, and $\mathrm{Al}$, where the $\mathrm{Al}$ range is from Sneden et al. (2004). A typical error bar is shown at the lower right. 
range of atomic number. The correlations among the elements showing such star-to-star variations suggest that $\mathrm{CN}$ and (especially for M13) ON burning, as well as NaMg burning, are required. These spreads have been widely discussed in the literature for the luminous giants in these and other Galactic GCs.

Our main contributions are twofold. First, we have confirmed with quite high precision that the sum of $\mathrm{C}+\mathrm{N}+\mathrm{O}$ is constant near the tip of the giant branch, as shown by Smith et al. (1996), but we extend this down to the bump in the luminosity function. Second, we have demonstrated that the correlations and anticorrelations among these light elements extend with the same amplitude seen on the upper RGB (but not with the extreme ratios characteristic of the tip of the RGB in M13) to the faintest luminosities probed, which for our sample in M13, is near the main-sequence turnoff. The range of luminosities for our sample in each of these two GCs reaches well below their RGB bump, which is at $V=15.45$ for M3 and at $V=14.75$ for M13 (Ferraro et al. 1999) and is where the first dredge-up is believed to begin. Stars less luminous than this cannot have mixed significantly, according to current theory (see, e.g., Charbonnel 1995; Pinsonneault 1997; Palacios et al. 2003).

This behavior demonstrates yet again that intrinsic nuclear processes within these low-mass, and in some cases relatively unevolved, stars is not capable of explaining the observed phenomena. In this context, we note the work of Gratton et al. (2000) on metal-poor field stars, which do not show these phenomena, but rather a much more orderly and smaller amplitude change in abundance ratios with luminosity. Thus, these phenomena seem restricted to globular clusters, where the stellar density is high and the gas density presumably also quite high in the past. Since internal nucleosynthesis has been ruled out, some form of external pollution onto the low-mass stars we currently see in GCs is required. This could be from a companion AGB star or from mass loss into the ISM of the cluster itself by higher mass stars presumably in the AGB phase, followed by accretion as the low-mass star passes through the denser regions near the cluster core, or by incomplete mixing of the cluster ISM prior to the formation of the generation of stars we see today. The problems with this general type of mechanism have been discussed at length by many (see, e.g., Cohen et al. 2002) and basically involve whether enough mass can be accreted, whether with the additional accreted material the observed abundance ratios can be reproduced (see, e.g., Fenner et al. 2004 for a discussion regarding massive AGB stars), and over what mass zones and how deeply down from the surface does the accreting star mix and hence dilute the accreted material.

All this exotic behavior ceases with $\mathrm{Si}$, whose abundance appears to be constant, with a small range consistent with the observational and modeling uncertainties. The regime from $\mathrm{Si}$ through the Fe peak is characterized by no detectable star-tostar variations and by strong overdepletion of the odd atomic number elements, expected for Fe-peak nuclei from considerations of explosive nucleosynthesis (Arnett 1971, 1996, p. 275). (Recall that among the lightest elements, the odd atomic numbers show large enhancements in $[\mathrm{X} / \mathrm{Fe}]$.)

The heaviest elements are not well sampled by our data. However, the $[\mathrm{Ba} / \mathrm{Eu}]$ ratio is $-0.35(-0.31)$ dex in M3 (M13), which value is intermediate between the solar ratio and that of the pure $r$-process, presumably reflecting the increased dominance of the $r$-process contribution at low metallicities.

The heaviest elements also show the first signs of star-to-star variations again, not surprising since their abundances $\mathrm{X} / \mathrm{H}$ are so low that any small addition of material could raise $[\mathrm{X} / \mathrm{Fe}]$.
In particular, M13 I-5 shows strong excesses of Y and of Ba, presumably from an $s$-process event, but we need, and have already obtained, better spectra with more complete wavelength coverage to verify this. Ignoring $\omega \mathrm{Cen},{ }^{8}$ star-to-star variations among the heavy elements have been previously detected only in M15 (Sneden et al. 1997), and there they appear quite different in character.

\section{THE EVOLUTION OF ABUNDANCES WITHIN THE GC SYSTEM}

While it is clear that the GCs differ from the field stars in the amplitude of their star-to-star variation of the light elements, we need to establish whether this difference persists in their average abundance ratios. If so, this would provide evidence for a difference in the chemical history and/or formation mechanisms for globular clusters from those of the halo field stars. With the advent of our program at the Keck Observatory and similar programs at ESO using UVES, there are now a substantial number of Galactic GCs for which detailed abundance analyses using high-precision, high-resolution spectra have been published. We collect those carried out at Keck and at the VLT and add in only recent analyses of relatively nearby GCs using $4 \mathrm{~m}$ telescopes. We impose a minimum of four stars per cluster. Ignoring the GCs associated with the Sgr dwarf galaxy, we find 13 clusters with suitable analyses, which, in order of decreasing metallicity, are NGC 6528 (Carretta et al. 2001), NGC 6553 (Cohen et al. 1999; Carretta et al. 2001), 47 Tuc (Carretta et al. 2004b; James et al. 2004b), M71 (Ramírez \& Cohen 2002, 2003), M5 (Ramírez \& Cohen 2003), NGC 288 (Shetrone \& Keane 2000), NGC 362 (Shetrone \& Keane 2000), NGC 6752 (James et al. 2004a), M3 (this paper; see also Sneden et al. 2004), M13 (this paper; see also Sneden et al. 2004), NGC 7492 (Cohen \& Melendez 2004), NGC 6397 (Thévenin et al. 2001; Gratton et al. 2001; James et al. 2004b), and M15 (Sneden et al. 1997). There are at least 10 more Galactic GCs that have been observed at Keck or at the VLT within the past 2 years with analyses in progress, so a significant fraction of the total Galactic population of GCs has been covered.

This strict selection of GC analyses guarantees the maximum possible accuracy of the abundance ratios, without, of course, guaranteeing consistency between the various analyses. Although the first author has been associated with seven of the 13, we have not tried to homogenize the details of the procedures adopted by other groups.

To characterize the behavior of the metal-poor halo field stars, we adopt abundance ratios from recent large surveys of such by Gratton \& Sneden (1991), McWilliam et al. (1995), Fulbright (2000), Nissen et al. (2000), and Johnson (2002). No effort has been made to homogenize these analyses either, but since they were carried out over the course of more than a decade, we have corrected for the difference in the solar Fe abundance adopted by each.

For both the field-star surveys and the GCs, we have looked at the differences between the transition probabilities used for each of these analyses and set them to the same absolute scale. Such differences are small for most species but are large (up to $0.2 \mathrm{dex}$ ) for Ca I, with substantial scatter in the difference from line to line.

\footnotetext{
${ }^{8}$ We ignore $\omega$ Cen in discussing star-to-star variations among globular clusters for the rest of this paper as it has been known for more than 20 years to have a wide range of heavy-element abundances (see, e.g., the latest such work, Pancino et al. 2002) and has been repeatedly suggested recently as the remnant of the nucleus of an accreted dwarf galaxy.
} 


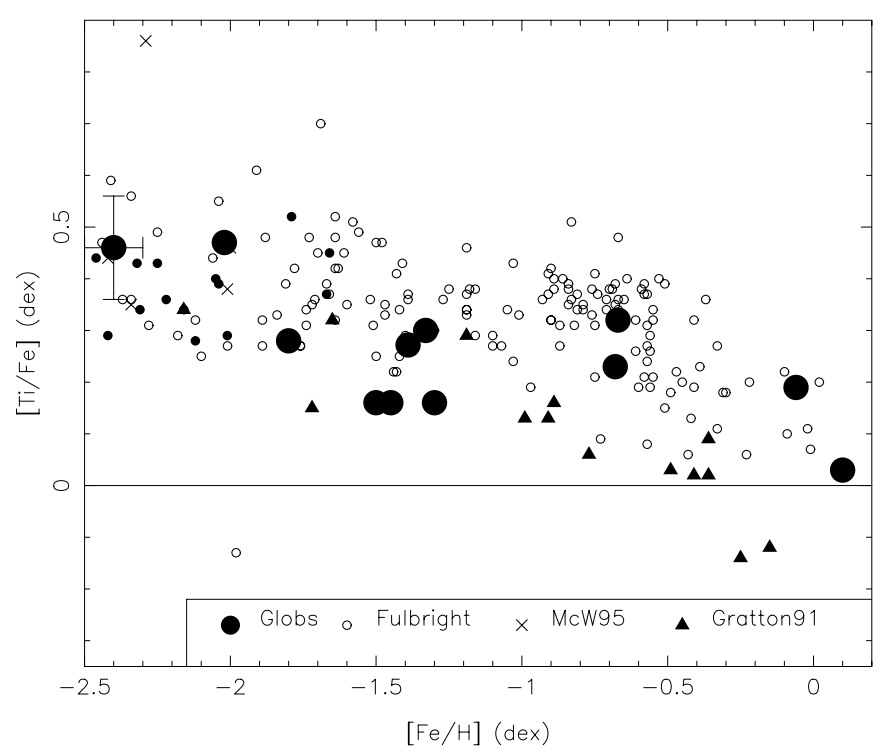

FIG. 21.-Abundance ratio $[\mathrm{Ti} / \mathrm{Fe}]$ shown as a function of $[\mathrm{Fe} / \mathrm{H}]$ for a sample of 13 Galactic GCs (see text for references), indicated as large filled circles. This is compared with the same relationship for halo field stars (sources and symbols indicated on the figure). An error bar typical of the GCs is shown for the lowest-metallicity GC.

When one examines the resulting relations between $[\mathrm{X} / \mathrm{H}]$ and $[\mathrm{Fe} / \mathrm{H}]$ for the Galactic globular clusters and for the halo field stars, one is struck by the similarity for many elements of the trends between the two systems, present in spite of completely independent analyses on very different samples (the halo stars being mostly dwarfs, while the GC samples focus on giants in many cases). Two typical cases are illustrated; for $\mathrm{Ti}$ (Fig. 21) and for Ba (Fig. 22), as for most elements, the relations of abundance ratio with metallicity of the GCs and of the halo field are indistinguishable.

The most credible difference we notice is shown by the $\alpha$-elements $\mathrm{Mg}$ and $\mathrm{Ca}$ (we show that of $[\mathrm{Ca} / \mathrm{Fe}]$ vs. $[\mathrm{Fe} / \mathrm{H}]$ in Fig. 23). The most metal-poor GC appears to be deficient in $\mathrm{Mg}$ and in $\mathrm{Ca}$ at $[\mathrm{Fe} / \mathrm{H}] \leq-1.5$ dex by $\sim 0.3$ dex as compared with the field. One explanation for this could be that the luminous giants usually analyzed in the GCs are slightly depleted in $\mathrm{Mg}$, an element that shows modest star-to-star variations in GCs, or perhaps non-LTE effects in Mg are playing a role. The notoriously uncertain $\mathrm{Mg} g f$-values could also be relevant if the same lines are not used by all the groups, and there are also problems in the $g f$-values for $\mathrm{Ca}$ I at the level of $\sim 0.2 \mathrm{dex}$, which may not have been completely removed. This difference in behavior between the GCs and the halo field is at present smaller than $2 \sigma$ and involves just the most metal-poor GC. We await publication of analyses for more of the most metal-deficient halo GCs to confirm the reality of this potential difference.

\section{SUMMARY}

We have carried a detailed abundance analysis for 21 elements in a sample of 25 stars with a wide range in luminosity from luminous giants to stars near the main-sequence turnoff in the globular cluster $\mathrm{M} 13([\mathrm{Fe} / \mathrm{H}]-1.50 \mathrm{dex})$ and in a sample of 13 stars distributed from the tip to the base of the RGB in the globular cluster M3 $([\mathrm{Fe} / \mathrm{H}]-1.39$ dex $)$. The analyzed spectra, obtained with HIRES at the Keck Observatory, are of high dispersion $(R=\lambda / \Delta \lambda=35,000)$. Most elements, including $\mathrm{Fe}$, but excluding the elements lighter than $\mathrm{Si}$, show no trend in abundance ratio $[\mathrm{X} / \mathrm{Fe}]$ with $T_{\mathrm{eff}}$ and scatter around the mean

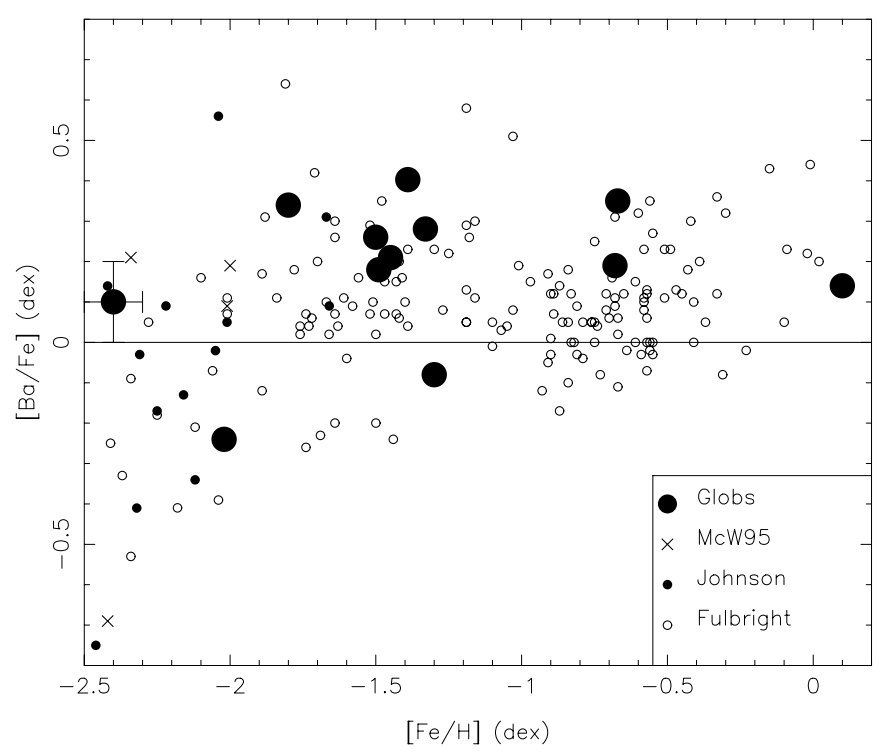

FIG. 22.-Same as Fig. 21, but for $[\mathrm{Ba} / \mathrm{Fe}]$.

between the top of the RGB and near the main-sequence turnoff, consistent with observational uncertainties. This suggests that at this metallicity, non-LTE effects and gravitationally induced heavy-element diffusion are not important for this set of elements over the range of stellar parameters spanned by our sample.

The elements lighter than Si that have been studied in detail, i.e., C and N (by Briley et al. 2002, 2004), O, Na, Mg, and Al (see Sneden et al. 2004), all show strong star-to-star variations and correlations among each other. We have detected an anticorrelation between $\mathrm{O}$ and $\mathrm{Na}$ abundances, observed previously only among the most luminous RGB stars in both of these clusters. We find these anticorrelations to persist in both M3 and in M13 over the full range of luminosity of our samples, i.e., in the case of M13 to near the main-sequence turnoff. M13 shows a larger range in both $\mathrm{O}$ and $\mathrm{Na}$ abundance than does $\mathrm{M} 3$ at all luminosities, in particular having a few stars at its RGB tip with very strongly depleted $\mathrm{O}$.

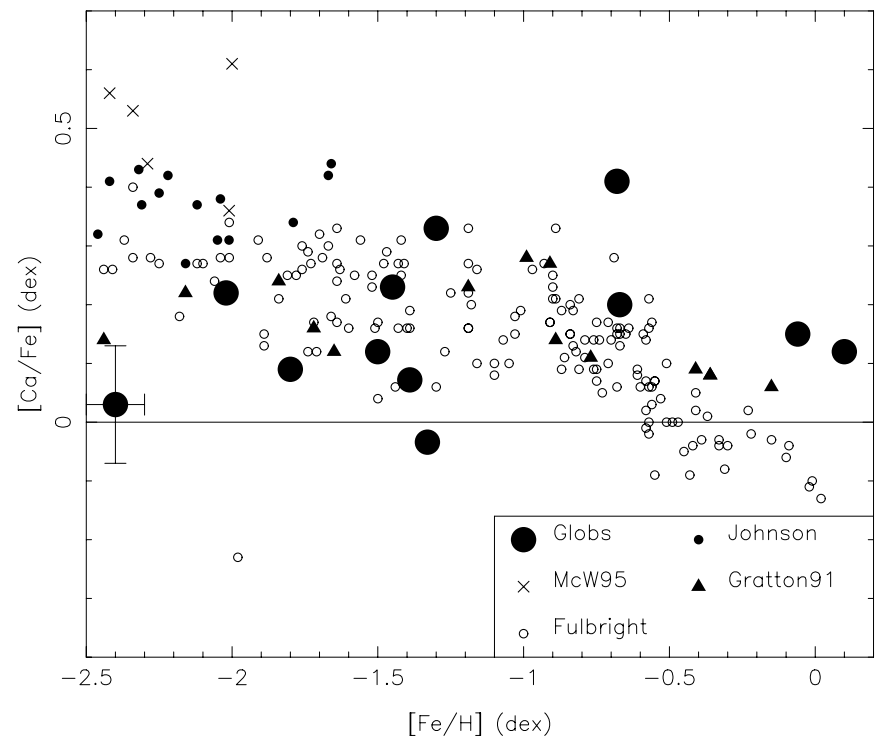

Fig. 23. - Same as Fig. 21, but for $[\mathrm{Ca} / \mathrm{Fe}]$. The $g f$-values adopted by the various groups have been adjusted to a common absolute scale. 
We detect a correlation between $\mathrm{Mg}$ abundance and $\mathrm{O}$ abundance among the stars in the M13 sample but no credible starto-star variation in $[\mathrm{Mg} / \mathrm{Fe}]$ within the $\mathrm{M} 3$ sample. We also find a decrease in the mean $\mathrm{Mg}$ abundance as one moves toward lower luminosity, which we tentatively suggest is due to ignoring non-LTE effects in $\mathrm{Mg}$.

Although $\mathrm{CN}$ burning must be occurring in both $\mathrm{M} 3$ and in M13 and ON burning is required for M13, we have confirmed with quite high precision that the sum of $\mathrm{C}+\mathrm{N}+\mathrm{O}$ is constant, $\log [\epsilon(\mathrm{C}+\mathrm{N}+\mathrm{O})]=-1.24$ dex with a relatively small $\sigma$ of 0.12 dex, as previously shown near the tip of the giant branch by Smith et al. (1996) for luminous giants, but we extend this down to the bump in the luminosity function. The same holds true for a smaller sample in M3, with somewhat larger variance.

We have shown that these star-to-star abundance variations among the light elements continue well below the RGB bump in both M3 and M13. The low luminosity at which these phenomena are now detected in M3 and M13 and, from our previous work, in M71 and in M5 (Ramírez \& Cohen 2003), as in other published analyses (see, e.g., Gratton et al. 2001; Carretta et al. 2004b), has effectively ruled out the possibility of generating the spreads through internal nucleosynthesis and mixing within the stars we observe today. Instead, some form of external pollution involving a previous generation of stars, combined with binaries or accretion of gas from the cluster ISM, must be involved. But, as discussed most recently by Fenner et al. (2004), the details do not fit (yet).

Star I-5 in M13 has large excesses of Y and of Ba, with no strong enhancement of Eu, suggesting that an $s$-process event contributed to its heavy-element abundances. This is the first star we have found in the present long-term effort that shows any credible deviation from the cluster mean for any heavy element.

The mean abundance ratios we derive for M3 and for M13 are identical to within the errors. They show the typical pattern of scatter among the light elements, with the odd atomic number elements appearing enhanced, as well as no star-to-star variations among the Fe-peak elements, where the odd atomic number elements are excessively depleted. The mean $[\mathrm{Eu} / \mathrm{Ba}]$ ratio is essentially the same in both clusters; it is intermediate between the solar ratio and that of the $r$-process, suggesting the additional $r$-process contribution characteristic of metal-poor populations. It does not appear possible to explain the significant differences in horizontal-branch characteristics of M3 and $\mathrm{M} 13$, the classic second-parameter pair, through differences in abundances (unless $\mathrm{He}$ is the culprit), since we have shown that these two clusters have essentially identical values of $[\mathrm{Fe} / \mathrm{H}]$ and of mean $[\mathrm{X} / \mathrm{Fe}]$ for all elements studied here.

The abundance ratios for 13 Galactic globular clusters with recent detailed abundance analyses, obtained by combining our samples with published data, are compared with those of published large surveys of metal-poor halo field stars. For most elements, the agreement is very good, suggesting a common chemical history for the halo field and for the Galactic globular clusters.

We see yet again that the abundances of the Fe-peak elements in M3 and in M13, which are rather massive globular clusters $\left(\sim 6 \times 10^{5} M_{\odot}\right)$ are single valued, with extremely narrow peaks. It is ironic that such intense theoretical and observational effort has in recent years focused on the correlations and anticorrelations among the light elements $\mathrm{C}, \mathrm{N}, \mathrm{O}, \mathrm{Na}, \mathrm{Mg}$, and $\mathrm{Al}$ in globular clusters and the mixing and nuclear processes that might produce these, and so little has focused on the amazingly narrow range of abundances of the Fe-peak elements characteristic of the Galactic globular clusters.

The entire Keck/HIRES user community owes a huge debt to Jerry Nelson, Gerry Smith, Steve Vogt, and many other people who have worked to make the Keck Telescope and HIRES a reality and to operate and maintain the Keck Observatory. This publication makes use of data from the Two Micron All-Sky Survey, which is a joint project of the University of Massachusetts and the Infrared Processing and Analysis Center, funded by NASA and the National Science Foundation (NSF). We are grateful to the NSF for partial support under grant AST 02-05951 to J. G. C.

\section{REFERENCES}

Allende Prieto, C., Barklem, P. S., Lambert, D. L., \& Cunha, K. 2004, A\&A, 420,183

Allende Prieto, C., Lambert, D. L., \& Asplund, M. 2001, ApJ, 556, L63

- 2002, ApJ, 573, L137

Anders, E., \& Grevesse, N. 1989, Geochim. Cosmochim. Acta, 53, 197

Arnett, D. 1996, Supernovae and Nucleosynthesis (Princeton: Princeton Univ. Press), 275

Arnett, W. D. 1971, ApJ, 166, 153

Arp, H. C. 1955, AJ, 60, 317

Asplund, M. 2003, in ASP Conf. Ser. 304, CNO in the Universe, ed. G. Charbonnel, D. Schaerer, \& G. Meynet (San Francisco: ASP), 275

Asplund, M., Grevesse, N., Sauval, A. J., Allende Prieto, C., \& Kiselman, D. 2004, A\&A, 417, 751

Asplund, M., Nordlund, A., Trampedach, R., Prieto, C. A., \& Stein, R. F. 2000, A\&A, 359, 729

Bard, A., \& Kock, M. 1994, A\&A, 282, 1014

Bard, A., Kock, A., \& Kock, M. 1991, A\&A, 248, 315

Barklem, P. S., Anstee, S. D., \& O’Mara, B. J. 1998, Publ. Astron. Soc. Australia, 15, 336

Barklem, P. S., Piskunov, N., \& O’Mara, B. J. 2000, A\&AS, 142, 467

Baumüller, D., \& Gehren, T. 1997, A\&A, 325, 1088

Bell, R. A., \& Dickens, R. J. 1980, ApJ, 242, 657

Biémont, E., Baudoux, M., Kurucz, R. L., Ansbacher, W., \& Pinnimgton, E. H. 1991, A\&A, 249, 539

Blackwell, D. E., Booth, A. J., Haddock, D. J., Petford, A. D., \& Leggett, S. K. 1986, MNRAS, 220, 549

Blackwell, D. E., Petford, A. D., \& Shallis, M. J. 1979, MNRAS, 186, 657

Blackwell, D. E., Petford, A. D., Shallis, M. J., \& Simmons, G. J. 1982a, MNRAS, 199, 43

Blackwell, D. E., Petford, A. D., \& Simmons, G. J. 1982b, MNRAS, 201, 595

Blackwell, D. E., Shallis, M. J., \& Simmons, G. J. 1980, A\&A, 81, 340

Briley, M. M., Cohen, J. G., \& Stetson, P. B. 2002, ApJ, 579, L17 2004, AJ, 127, 1579

Brown, J. A., Wallerstein, G., \& Oke, J. B. 1991, AJ, 101, 1693

Buonanno, R., Corsi, C. E., Buzzoni, A., Cacciari, C., Ferraro, F. R., \& Fusi Pecci, F. 1994, A\&A, 290, 69

Carretta, E., Bragaglia, A., \& Cacciari, C. 2004a, ApJ, 610, L25

Carretta, E., Cohen, J. G., Gratton, R. G., \& Behr, B. B. 2001, AJ, 122, 1469

Carretta, E., Gratton, R., Bragaglia, A., Bonifacio, P., \& Pasquini, L. 2004b, A\&A, 416, 925

Cavallo, R. M., \& Nagar, N. M. 2000, AJ, 120, 1364

Charbonnel, C. 1995, ApJ, 453, L41

Cohen, J. G. 1978, ApJ, 223, 487

Cohen, J. G., Behr, B. B., \& Briley, M. M. 2001, AJ, 122, 1420

Cohen, J. G., Briley, M. M., \& Stetson, P. B. 2002, AJ, 123, 2525

Cohen, J. G., Frogel, J. A., Persson, S. E., \& Elias, J. H. 1981, ApJ, 249, 481

Cohen, J. G., Gratton, R. G., Behr, B. B., \& Carretta, E. 1999, ApJ, 523, 739

Cohen, J. G., \& Melendez, J. 2004, AJ, submitted

Cudworth, K. C. 1979 , AJ, 84, 1312

Cudworth, K. C., \& Monet, D. M. 1979, AJ, 84, 774

Cutri, R. M., et al. 2003, Explanatory Supplement to the 2MASS All-Sky Data Release (Pasadena: IPAC), http://www.ipac.caltech.edu/2mass/releases/allsky/doc/explsup.html

Davidge, T. J., \& Courteau, S. 1999, AJ, 117, 1297 
Den Hartog, E. A., Lawler, J. E., Sneden, C., \& Cowan, J. J. 2003, ApJS, 148, 543

Fenner, Y. Campbell, S., Karakas, A. I., Lattanzio, J. C., \& Gibson, B. K. 2004, MNRAS, 353, 789

Ferraro, F. R., Carretta, E., Corsi, C. E., Fusi Pecci, F., Cacciari, C., Buonanno, R., Paltrinieri, B., \& Hamilton, D. 1997, A\&A, 320, 757

Ferraro, F. R., Messineo, M., Fusi Pecci, F., Da Palo, M. A. D., Straniero, O., Chieffi, A., \& Limongi, M. 1999, AJ, 118, 1738

Fuhr, J. R., Martin, G. A., \& Wiese, W. L. 1988, J. Phys. Chem. Ref. Data Suppl., 17, 4

Fulbright, J. P. 2000, AJ, 120, 1841

Gehren, T., Liang, Y. C., Shi, J. R., Zhang, H. W., \& Zhao, G. 2004, A\&A, 413, 1045

Gratton, R. G., Carretta, E., Eriksson, K., \& Gustafsson, B. 1999, A\&A, 350, 955

Gratton, R. G., \& Sneden, C. 1991, A\&A, 241, 501

Gratton, R. G., Sneden, C., Carretta, E., \& Bragaglia, A. 2000, A\&A, 354, 169

Gratton, R. G., et al. 2001, A\&A, 369, 87

Grevesse, N., \& Sauval, A. J. 1998, Space Sci. Rev., 85, 161

Gunn, J. E., \& Griffin, R. F. 1979, AJ, 84, 752

Gustafsson, B., Bell, R. A., Eriksson, K., \& Nordlund, A. 1975, A\&A, 42, 407

Harris, W. E. 1996, AJ, 112, 1487

Holweger, H. 2001, in AIP Conf. Proc. 598, Solar and Galactic Composition, ed. R. F. Wimmer-Schweingruber (Woodbury, NY: AIP), 23

Holweger, H., Bard, A., Kock, A., \& Kock, M. 1991, A\&A, 249, 545

Houdashelt, M. L., Bell, R. A., \& Sweigart, A. V. 2000, AJ, 119, 1448

James, G., et al. 2004a, A\&A, 414, 1071

James, G., François, P., Bonifacio, P., Carretta, E., Gratton, R. G., \& Spite, F. 2004b, A\&A, in press

Johnson, H. L., \& Sandage, A. R. 1956, ApJ, 124, 379

Johnson, J. A. 2002, ApJS, 139, 219

Johnson, J. A., \& Bolte, M. M. 1998, AJ, 115, 693

Kadla, Z. I. 1966, Izv. Gl. Astron. Obs. Pulkova, 24, No. 181, 93

Kraft, R. P. 1994, PASP, 106, 553

Kraft, R. P., Sneden, C., Langer, G. E., \& Prosser, C. F. 1992, AJ, 104, 645

Kraft, R. P., Sneden, C., Smith, G. H., Shetrone, M. D., Langer, G. E., \& Pilachowski, C. A. 1997, AJ, 113, 279

Kurucz, R. L. 1993a, CD-ROM 12, ATLAS9 Stellar Atmosphere Programs and $2 \mathrm{~km} / \mathrm{s}$ Grid (Cambridge: Smithsonian Astrophys. Obs.)

1993b, CD-ROM 18, SYNTHE Spectrum Synthesis Programs and Line Data (Cambridge: Smithsonian Astrophys. Obs.)

Kurucz, R. L., Furenlid, I., Brault, J., \& Testerman, L. 1984, Solar Flux Atlas From 296 to $1300 \mathrm{~nm}$, National Solar Observatory Atlas No. 1 (Sunspot: Natl. Sol. Obs.)

Lawler, J. E., Bonvallet, G., \& Sneden, C. 2001, ApJ, 556, 452

Lawler, J. E., Wickliffe, M. E., den Hartog, E. A., \& Sneden, C. 2001, ApJ, 563,1075

Lupton, R. H., Gunn, J. E., \& Griffin, R. F. 1987, AJ, 93, 1114

Martin, G. A., Fuhr, J. R., \& Wiese, W. L. 1988, J. Phys. Chem. Ref. Data Suppl. 17, 3

Mashonkina, L., \& Gehren, T. 2000, A\&A, 364, 249

Mashonkina, L., Gehren, T., \& Bikmaev, I. 1999, A\&A, 343, 519

May, M., Richter, J., \& Wichelmann, J. 1974, A\&AS, 18, 405

McWilliam, A. 1998, AJ, 115, 1640

McWilliam, A., Preston, G. W., Sneden, C., \& Searle, L. 1995, AJ, 109, 2757

Moore, C. E., Minnaert, M. G. J., \& Houtgast, J. 1966, The Solar Spectrum $2935 \AA$ to $8770 \AA$ (Washington: GPO)
Nissen, P. E., Chen, Y. Q., Schuster, W. J., \& Zhao, G. 2000, A\&A, 353, 722 O’Brian, T. R., Wickliffe, M. E., Lawler, J. E., Whaling, W., \& Brault, J. W. 1991, J. Opt. Soc. Am. B, 8, 1185

Palacios, A., Talon, S., Charbonnel, C., \& Forestini, M. 2003, A\&A, 399, 603

Pancino, E., Pasquini, L., Hill, V., Ferraro, F. R., \& Bellazzini, M. 2002, ApJ, 568, L101

Pinsonneault, M. 1997, ARA\&A, 35, 557

Piotto, G., et al. 2002, A\&A, 391, 945

Prochaska, J. X., Naumov, S. O., Carney, B. W., McWilliam, A., \& Wolfe, A. M. 2000, ApJ, 120, 2513

Pryor, C., Latham, D. W., \& Hazen, M. L. 1988, AJ, 96, 123

Ramírez, S. V., \& Cohen, J. G. 2002, AJ, 123, 3277 2003, AJ, 125, 224

Ramírez, S. V., Cohen, J. G., Buss, J., \& Briley, M. M. 2001, AJ, 122, 1429

Rey, S.-C., Yoon, S.-J., Lee, Y. W., Chaboyer, B., \& Sarajedini, A. 2001, AJ, 122,3219

Rosenberg, A., Aparicio, A., Saviane, I., \& Piotto, G. 2000, A\&AS, 145, 451

Sandage, A. R. 1953, AJ, 58, 61

Schlegel, D. J., Finkbeiner, D. P., \& Davis, M. 1998, ApJ, 500, 525

Shetrone, M., \& Keane, M. J. 2000, AJ, 119, 840

Shetrone, M. D. 1996, AJ, 112, 1517

Skrutskie, M. F., et al. 1997, in The Impact of Large Scale Near-IR Sky Surveys, ed. F. Garzon et al. (Dordrecht: Kluwer), 187

Smith, G. H., Shetrone, M. D., Bell, R. A., Churchill, C., \& Briley, M. M. 1996, AJ, 112, 1511

Sneden, C. 1973, Ph.D. thesis, Univ. Texas

Sneden, C., Kraft, R. P., Guhathakurta, P., Peterson, R. C., \& Fulbright, J. P. 2004, AJ, 127, 2162

Sneden, C., Kraft, R. P., Shetrone, M. D., Smith, G. H., Langer, G. E., \& Prosser, C. F. 1997, AJ, 114, 1964

Stetson, P. B. 2000, PASP, 112, 925

Stetson, P. B., Hesser, J. E., \& Smecker-Hane, T. 1998, PASP, 110, 533

Suntzeff, N. B. 1981, ApJS, 47, 1

Takeda, Y., Zhoa, G., Takada-Hidai, M., Chen, Y.-Q., Saito, Y., \& Zhang, H.-W. 2003, Chinese J. Astron. Astrophys., 3, 316

Thévenin, F. 1989, A\&AS, 77, 137

1990, A\&AS, 82, 179

Thévenin, F., Carbonnel, C., de Freitas Pacheco, J. A., Idiart, T. P., Jasniewsicz, G., de Lavery, P., \& Plez, B. 2001, A\&A, 373, 905

Valenti, E., Ferraro, F. R., Perina, S., \& Origlia, L. 2004, A\&A, 419, 139

Ventura, J., D’Antona, F., Mazzitelli, I., \& Gratton, R. 2001, ApJ, 550, L65

Vogt, S. E., et al. 1994, Proc. SPIE, 2198, 362

von Zeipel, M. H. 1908, Ann. Obs. Paris Mem., 25, F.1

Wallace, L., Hinkle, K., \& Livingston, W. C. 1998, An Atlas of the Spectrum of the Solar Photosphere from 13,500 to $28,000 \mathrm{~cm}^{-1}$ (3570 to $7405 \AA$ ) (NSO Tech. Rep. 98-001) (Tucson: Natl. Sol. Obs.), ftp://ftp.noao.edu/fts/visatl/ README

Weise, W. L., Fuhr, J. R., \& Deters, T. M. 1996, J. Phys. Chem. Ref. Data, Monog. 7

Weise, W. L., Smith, M. W., \& Miles, B. M. 1969, Atomic Transition Probabilities, Vol. 2: Sodium through Calcium (NSRDS-NBS 22) (Washington: Dept. Comm., Natl. Bur. Stand.)

Wilson, W. C., et al. 2003, Proc. SPIE, 484, 4157

Wolnik, S. J., Berthel, R. O., \& Wares, G. W. 1971, ApJ, 166, L31

Zhao, G., \& Gehren, T. 2000, A\&A, 362, 1077 